DOE/ER/40318--Tl

DE91 002762

\title{
HIGH ENERGY PHYSICS AT THE UNIVERSITY OF IOWA
}

\author{
Progress Report \\ for the Period 1 February 1990 - 31 January 1991 \\ Edward R. MCCrinient, Charles R. Newsom, \\ AND YASAR ONEL \\ Department of Physics and Astronomy \\ The University of lowa \\ lowa City, lowa 52242-1479
}

\begin{abstract}
NOTICE
This report was prepared as an account of work sponsored by the United States Government. Neither the United States nor the Department of Energy, nor any of their employees, nor any of their contractors, subcontractors, or their employees, makes any warranty, express or implied, or assumes any legal liability or responsibility for the accuracy, completeness, or usefulness of any information, apparatus, product or process disclosed or represents that its use would not infringe privately owned rights.
\end{abstract}

DEPARTMENT OF ENERGY

AGREEMENT NO. DE-AC02-87ER40318

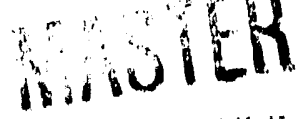


PART A. PROGRESS REPORT: HYPERON SPIN PHYSICS AT FERMI C. R. Newsom

I. Introduction ................... . 2

II. Silicon Detector Integration . . . . . . . . . . . . . . . . . . 2

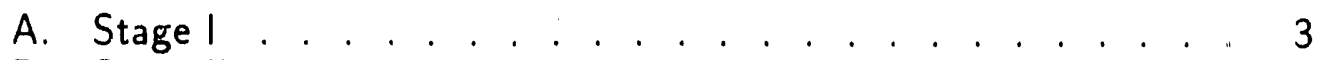

B. Stage II . . . . . . . . . . . . . . . . . . . . . 4

III. Data Acquisition Period . . . . . . . . . . . . . . . . . . . . . 5

A. The Transition Radiation Detector Apparatus . . . . . . . . 6

B. The Lead Glass Detector Apparatus . . . . . . . . . . . . 7

IV. Results . . . . . . . . . . . . . . . . . . . . . . . . 8

A. The Positive Hyperon Beam Decays . . . . . . . . . . . . . . . . . 9

B. Results Using the Negative Hyperon Beam . . . . . . . . . 12

V. Additional Polarization and Magnetic Moment Results . . . . . . . . 15

ソl. Summary . . . . . . . . . . . . . . . . . . . . . 17

VII. Expenditure of Contract Funds . . . . . . . . . . . . . . . . . . 19

PART B. PROGRESS REPORT: HADRONIC INTERACTIONS AT FERMILAB $Y$. Onel

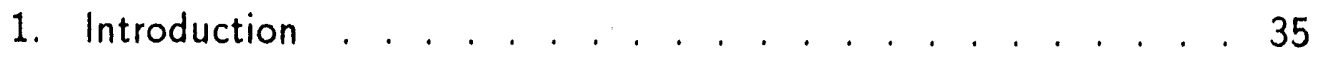

2. E-704 . . . . . . . . . . . . . . 36

2.а. CNI . . . . . . . . . . . 37

2.b. Measurements . . . . . . . . . . . . . . . . . . . 40

2.c. Other Progress in E-704 . . . . . . . . . . . . . . . 40 
3. E-683 ........................ 44

3.a. Progress on lowa Drift Chambers . . . . . . . . . . . 46

Expenditures . . . . . . . . . . . . . . . . . . . . 50

\section{PART C. PROGRESS REPORT}

\section{E. R. McCliment}

A. Introduction .................. . . . . . . . .

B. Work on Experiment E761-Hyperon Radiative Decay . . . . . 75

1. Magnet Scans (Tuneup) . . . . . . . . . . . . . . . . . 75

2. The Sigma Configuration . . . . . . . . . . . . . . . . . 75

3. Cascade Configuration . . . . . . . . . . . . . . . . . . 77

4. Preliminary Search for Radiative Cascade-Decays . . . . . . . 78

5. Background Monte Carlo Studies . . . . . . . . . . . . . 79

C. Work on BCD Silicon Detector Test Run ... . . . . . . . 80

D. Work on $B_{s}-B_{s}$ Bar Mixing . . . . . . . . . . . . . . . . 81

E. Work on the E781 Monte Carlo . . . . . . . . . . . . . 83

F. Work on E781 Hardware . . . . . . . . . . . . . 85

G. Summary . . . . . . . . . . . . . . 85

H. Expenditures . . . . . . . . . . . . . . 86

\section{DISCLAIMER}

This report was prepared as an account of work sponsored by an agency of the United States Government. Neither the United States Government nor any agency thereof, nor any of their employees, makes any warranty, express or implied, or assumes any legal liability or responsibility for the accuracy, completeness, or usefulness of any information, apparatus, product, or process disclosed, or represents that its use would not infringe privately owned rights. Reference herein to any specific commercial product, process, or service by trade name, trademark, manufacturer, or otherwise does not necessarily constitute or imply its endorsement, recommendation, or favoring by the United States Government or any agency thereof. The views and opinions of authors expressed herein do not necessarily state or reflect those of the United States Government or any agency thereof. 
PART A

\section{Progress Report}

Hyperon Spin Physics at Fermi

C. R. Newsom 


\section{Introduction}

During the past twelve months we have seen Experiment 761 change from a hope into a reality. At the end of the previous report, the silicon detectors had been installed, and the hardware checkout period had just begun. Since the lowa silicon detector system was by far the most advanced (in the schedule) of all of the detector systems, this system was seen by the group as the driving force behind the integration of the experiment. In order to read out the silicon, it was necessary to develop the trigger, and debug the data acquisition system software. since our data acquisition system consisted of programmable camac modules, programmable "smart" crate controllers, and programmable ACP nodes, this was no small task. Approximately 2 months after the silicon system was first read-out by the full DAQ system, the hyperon beam became available and the experiment began in earnest. The initial beam period was spent in checking out the entire apparatus, and setting up a viable physics trigger, based on actual background rates. The final beam period covered by this report was spent in on-line analysis. I will describe my contributions to each of these efforts in more detail below.

\section{Silicon Detector Integration}

The integration of the silicon detector system into the on-line acquisition system proceeded in two stages. First, an IBM-PC based readout system designed by the Russians was installed and tested. Since it was originally designed to test multi wire proportional chambers, some modifications, under my supervision, were necessary. The purpose of this system was to read out the slilicon detectors and the wire chambers in a relatively simple fashion, using a computer generated trigger

pulse. Both the timing and pulse height were controlled by the 
computer. We were thus able to trouble-shoot hardware problems in a straightforward way. In the second stage, the trigger and the real acquisition system were turned on and tested. The need for two independent systems was due to the complexity of our readout system, and the fact that most of our read-out electronics was newly designed and built in Leningrad, and thus it was necessary to spend some time learning about the system.

\section{A. Stage 1}

The PC based system was used for 3 purposes. First, we used it to identify dead channels in the silicon system. This was done by sending an analog pulse to the HV plane of the silicon detector. The resultant output would appear simultaneously on all 1000 channels of the plane being tested. The efficiency of the readout was then measured as a function of the analog pulse height, and thresholds plotted for all channels (figure 1). This was especially useful in identifying bad readout channels. These were due primarily to bad amplifier cards, which were then replaced. Some bad pre-amplifiers were also replaced, as well as a few bad cables. After repairing the dead channels, we proceeded to check out the timing of each channel. This was done by varying the timing between the trigger pulse and the camac strobe. The efficiency of the readout was then recorded as a function of delay, to yield the best timing curve for all 1000 channels simultaneously. (Note, 1000 channels refers to a maximally instrumented plane. Most silicon planes did not require full instrumentation.) The efficiency and timing measurements were fully automated and required approximately 1-2 minutes per plane. Ir addition to the above tests, the Russians also used the IBM-PC to strobe the camac directly, thus identifying any bad camac 
modules directly. This was done on a daily basis by the Russians for approximately I year!

\section{B. Stage 11}

Once the electronics were fully checked out, we then proceeded to integrate this with the ACP/VAX based MULTI driven readout system. (MULTI has been used at FNAL for many years for reading out experiments.) The initial data was strobed into in the Camac electronics by the first level beam scintillator trigger. A second strobe would then be sent to the electronics by the trigger, which would begin transferring the data from the camac via camac controllers to Russian branch drivers and buffers. There were two branches on the system, controlling the silicon and MWPC systems. The data would then be read into a smart crate controller, and finally stored in an ACP node. From there, it would be written onto magnetic tape. The initial NIM based trigger was constructed by A. Morelos, the Camac trigger by M. Foucher, and A. Denisov, and the SCC and Russian controller software was written by A. Morelos. My primary task during the startup period was to organize the integration of the work of all of these pieces into a working whole. The primary difficulty that we encountered was understanding the detailed workings of the Russian electronics. This was partly due to the fact that it had never been used in an experiment before, and thus both we and the Russians were discovering the unusual "features" of this system. Other problems arose due to the complex computer-computer handshaking that was taking place at many levels simultaneously. This task was completed in approximately 6 weeks, and began working about 2 months before the hyperon beam was delivered to Proton Center. 


\section{Data Acquisition Period}

The beam first arrived in proton center in the beginning of February 1989. Since I was teaching during this semester, it was necessary fol to commute between Fermilab and lowa. Most of this period was spent in debugging the other major detector systems, and in a sense, did not. require my intensive presence (thankfully). At the end of my teaching, in early May, I returned to Fermilab, and resumed working full time on the experiment.

Upon my return, it was clear that the hardware and trigger checkout phase of the experiment was now basically complete. I thus concentrated my efforts upon data analysis. The main goal of this phase of the experiment was to measure the branching ratio and asymmetry for the reaction $\Sigma^{+} \gg p+\gamma$. Specifically, I took on the task of separating the $\Sigma^{+} \gg p+\gamma$, signal, from the $\Sigma^{+} \gg p+\pi^{0}$ background. When I began, the signal was approximately 1:100 (figure 2). The status of the analysis was that all of the charged particle information (tracking, momentum, etc) had been utilized to its fullest. The only improve that I was able to make to this part of the analysis was to calculate the distance of closest approach (DCA) between the two charged tracks. This proved a useful cut for eliminating uncorrelated tracks (figure 3). The next logical step was to use the photon information to improve the signal to background. The major goal was to identify a clear signal, in order to determine how much data had been written to tape, and to tell us when this phase of the experiment was complete. 
A. The Transition Radlation Detector Apparatus

The transition radiation detector (TRD) apparatus consisted of 3 parts (figure 4). First, was a preradiator, which converted the photons into charged particles. Next, the charged particles (mostly electronpositron pairs) were then detected by the TRDs. Finally, a simple proportional wire chamber (PWC) was added which was efficient for all charged particles. The full TRD apparatus consisted of 2 identical sets of radiator/PWC/TRD detectors, placed one behind the other, for maximum photon detector efficlency. scintillators were also added before and after each radiator and were used for fast triggering. This phase of the analysis was begun as a collaborative effort between A. Vorobyov, and myself, with T. Shoppa replacing Vorobyov, upon his departure 2 weeks later. Initially, we took the tracking information from both the TRDs and the PWCs, and compared this with the projected neutral track. This projection was obtained by subtracting the vector momentum of the outgoing charged track from the incoming charged track. This defined the direction of the "missing momentum". The first algorithm that was tried simply required all $x$ and $y$ information ( 2 of 2 TRD, and 2 of 2 PWC planes) be present and that a hit occur within an acceptance window. Although this algorithm will lose lots of data, it also provides a very "clean" sample. This was our highest priority. The results of this analysis were obtained around midnight one sunday, and shown at the Monday users meeting, the next day. Our signal to background had improved from 1:100 to about 3:1 (figure 5). From this "clean" sample of events, many members of the group were then able check many aspects of the experiment, and indeed, several serious problems were found and corrected in the on-line analysis code. 
Now that a clear signal had been seen, it was necessary to try to estimate the total signal that had been written to tape. This would enable us to determine when enough data had been acquired, thus allowing us to continue on into the next phase of the experiment. Thus, we began working on better TRD algorithms. Many different algorithms were tried, and the best algorithm that has been found so far was to allow any one plane to fall outside our acceptance "window". (Although this seems rather obvious, it should be pointed out that our signal is buried within a shower, and each wire of the TRD gave us the number of clusters that had hit that wire.) With this algorithm, the overall reconstruction efficiency of the signal improved by about a factor of 2 . By ignoring the PWC information for one plane, we could see that the PWC efficiencies were about $80 \%$ for our photon tracks. Thus we measured the (TRD cart) PWC efficiencies using $\Sigma^{+} \gg p+\gamma$ radiative decay eventsl

\section{B. The Lead Glass Detector Apparatus}

The TRD detector in the experiment was followed by an array of lead glass blocks. The position information from the TRD detector could then be combiried with the energy measured in the lead glass yielding complete neutral track information. The energy resolution of the lead glass detector was about 22\% at the beginning of this study. This was mainly due to the fact that no one had spent much time optimizing the gain information in the analysis code. I wrote an analysis routine which relied on the $\Sigma^{+} \gg p+\pi^{0}$ background for calibration information. After a few days of debugging and analysis, the energy resolution had been improved to about $7 \%$ (figure 6). To improve the resolution further, one would 
have to handle the problem of energy leakage between the blocks, and out the back of the detector. However, this resolution served to improve the analysis in several ways. First, for both the signal and our primary background, E/P is expected to be 1.0. By cutting on E/P, all events which do not produce zero mass photons, are rejected to a large degree. Secondly, if one of the two photons from a $\Sigma^{+} \gg p+\pi^{0}$ decay or a $K^{+} \gg \pi^{+}+\pi^{0}$ misses the lead glass, then E/P Will be less than one and thus some rejection will occur.

Finally, it was necessary to eliminate those events in which two photons had been detected. I requested $P$. Roth, an lowa computer science major to write the pattern recognition program. After modifications, this program was installed in the analysis. Basically, the detector was divided into two halves, with a dead zone between. The Leit/Right and Top/Bottom energy asymmetries were then each calculated, with the largest asymmetry being recorded. A one photon event would then have an asymmetry near \pm 1.0 , with two photon events spread about zero. This worked very well, and the results can be seen in figure 7 .

\section{Results}

The experiment was done in two parts. The first half of our beam time was allocated to taking data with a positive hyperon beam. In this part of the experiment, two decays were of primary interest, namely, $\Sigma^{+} \gg p+\gamma$ and $\Sigma^{+} \gg p+\pi^{0}$. Any other decays are either too weak to be of any interest, or useful only as a zero-asymmetry verification, for systematics studies.

The second half of our beam allocation was given over to negative hyperon decays. In this case, it was 
necessary to move the downstream spectrometers nearer to the sllicon spectrometer. This was done because the negative beam decay products are themselves unstable. The primary decays that we studied were $\Xi^{-} \gg \Sigma^{-}+\gamma$ radiative: decays and anti( $\left.\Sigma^{+} \gg p+\pi^{0}\right)$.

\section{A The positive hyperon beam decays}

The positive hyperon beam consisted mainly of protons. The $\Sigma^{+}$hyperons constituted about $2 \%$ of the beam (table 1). In order to eliminate the majority of the beam triggers, it was necessary to trigger on decays producing one or more photons. Fortunately, aue to a long kajn laboratory lifetime, few kaon decays occur within the decay volume. Thus, this reaction did not provide a significant trigger rate. Once the trigger rate was below a certain level, all events were accepted for later analysis.

\section{IABLE 1}

\section{POSITIVE BEAM COMPOSITION}

\begin{tabular}{|cc|}
\hline$P$ & $60 \%$ \\
$\pi^{+}$ & $30 \%$ \\
$K^{+}$ & $6 \%$ \\
$\Sigma^{+}$ & $2 \%$ \\
$\operatorname{Anti}\left(\Sigma^{-}\right)$ & $.15 \%$ \\
$\operatorname{Anti}(\Xi-)$ & $.03 \%$ \\
$\operatorname{Anti}\left(\Omega^{-}\right)$ & $.000006 \%$ \\
\hline
\end{tabular}


The original purpose of this part of the experiment was to measure the branching ratio and asymmetry of the $\Sigma^{+} \gg p+\gamma$ radiative decay. The primary background is due to $\Sigma^{+} \gg p+\pi^{0}$ decays, and is 1000 times larger than the radiative decay events. Fortunately, the background is also useful, since it can be used to obtain the polarization of the $\Sigma^{+}$beam. Initially, 8 tapes of data were sufficient to surpass the (polarized) world sampie of the $\Sigma^{+} \gg p+\pi^{0}$ events. Approximately $217 \mathrm{~K}$ $\Sigma^{+} \gg p+\pi^{0}$ events have been reconstructed from these tapes, representing about $1 \%$ of the total data sample (figure 2). Clearly, our results will be limited by systematics when the full data sample is analyzed. Preliminary asymmetries for $\Sigma^{+} \gg p+\pi^{0}$ are shown in figures 8,9 , and 10 . Since our beain was produced by scattering a proton from a copper target in the horizontal plane, one expects the resultant polarization to be in the vertical ( $Y$ ) plane. From the figures, one can clearly see that the $X$ and $Z$ asymmetries are consistent with zero, as expected, and the $Y$ asymmetry is clearly nonzero. Preliminary asymmeiries $(\alpha P)$ at a lab angle of 3 milliradians and hyperon momentum of $370 \mathrm{GeV} / \mathrm{c}$ are presented in table 2. With an alpha parameter of $0.98(2)$, this represents a beam polarization of $11.4(4) \%$. The errors show here are purely statistical. This is the first reported result of the $\Sigma^{+}$beam polarization at using an $800 \mathrm{GeV}$ proton beam.

The on-line analysis of the data consisted of analyzing one tape for each run. Each run consisted of 3 to 5 tapes. Thus about one fourth of the data was available for additional off-line analysis. After analyzing approximately 231 tapes of data, approximately $4000 \Sigma^{+} \gg p+\gamma$ events were reconstructed (figure 4 ). The signal to background for 
these events is approximately 20:1. A careful study of

\section{Table 2}

$\frac{\text { Asymmetries for the reaction: }}{\Sigma^{+}>p+\pi^{0}}$

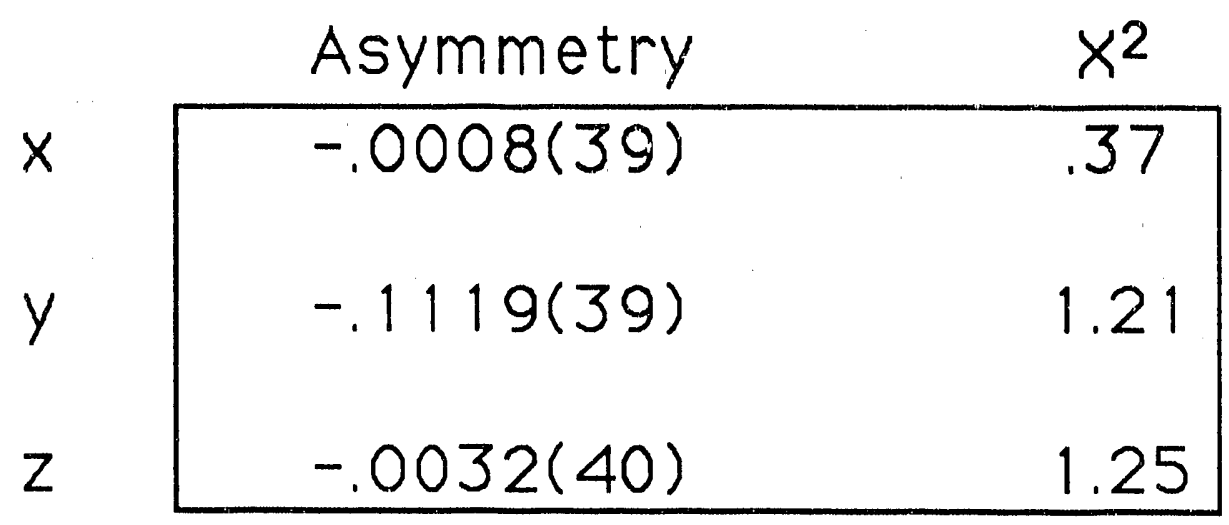

the cut efficiencies indicates that the cuts are eliminating about $50 \%$ of the data. A summary of the efiects of the cuts on the radiative decay signal is shown in table 3 . The signal column contains the number of fitted events in the peak at zero (photon) mass. The ratio in the right hand column represents the loss of events due to a given cut.

Careful studies by myself and $T$. Shoppa indicate that the track reconstruction efficiency for $\Sigma^{+} \gg p+\gamma$ events could be improved by about a factor of 2.2(2). This was accomplished by using a very slow, but efficient track reconstruction algorithm written by me in the test run, two years ago, ard adapted by $P$. Gouffon for use in the detector alignment software. Wich optimized cuts, we expect to reconstruct about 40-50K clean $\Sigma^{+} \gg p+\gamma$ events, representing a sample more than 2 orders of magnitude larger than the world data sample. The asymmetries have been calculated, and a 
careful study of the systematic errors is presently underway. From the above analysis, the statistical error in the alpha parameter for this reaction is \pm 0.23 . Using the fuil data sample, optimized cuts, and the better fitting algorithm, one could expect a final statistical error of about $\pm 0,06$. The results should be available in a few months.

\section{Table 3}

\section{Effects of removing one cut on the Sigma to $p+$ gamma signal.}

All Cuts

No chisq

No trd

No chi2_y

No zVertex

No chi2_b

No EoverP

No Ph Space

No Locsum

No Kaon

No Rpbg

No DCa
Signal Error Peak S/B Ratio

\begin{tabular}{|cc|c|c|}
\hline 1975 & 75 & 19.7 & - \\
2785 & 88 & 10 & $41.0 \%$ \\
2585 & 94 & 12.4 & $30.9 \%$ \\
2439 & 80 & 13 & $23.5 \%$ \\
2268 & 81 & 16.7 & $14.8 \%$ \\
2180 & 81 & 13.4 & $10.4 \%$ \\
2116 & 82 & 17.5 & $7.2 \%$ \\
2090 & 78 & 17.2 & $5.8 \%$ \\
2084 & 82 & 13 & $5.5 \%$ \\
2068 & 79 & 15.9 & $4.7 \%$ \\
1995 & 76 & 18.3 & $1.0 \%$ \\
1975 & 75 & 17.5 & $0.0 \%$ \\
\hline
\end{tabular}

\section{B. Results using the negative hyperon beam}

The second half of experiment 761 consisted of moving most of the detectors (excluding the silicon) upstream, and changing the polarity of the hyperon and spectrometer magnets. The entire procedure was done in 2 days! checkout of the apparatus then took about 2 weeks, including the studies of the negative backgrounds. 
The approximate makeup of the negative beam is shown in Table 4.

\section{IABLE 4}

NEGATIVE BEAM COMPOSITION

\begin{tabular}{|cc|}
\hline$\pi-$ & $70 \%$ \\
$\Sigma-$ & $15 \%$ \\
$K-$ & $6 \%$ \\
$\Xi$ & $2 \%$ \\
Anti(P) & $1.5 \%$ \\
Anti( $\left.\Sigma^{+}\right)$ & $.03 \%$ \\
$\Omega-$ & $.00009 \%$ \\
\hline
\end{tabular}

The two decays of primary interest in the negative beam are $\Xi^{-} \gg \Sigma^{-}+\gamma$, and $\operatorname{Anti}\left(\Sigma^{+} \gg p+\pi^{0}\right)$. The cascade radiative decays represent about 1 part in $10,000,000$ of the total beam decays. Because of the extremely small signal to background ratio, it was necessary to create a very effirient trigger. The most important aspect of the trigger was the photon requirement. From the above table, the 2 most prolific beam particles do not of ten decay into $\pi^{0} \mathrm{~s}$ which in turn decay into photons. The energetic kaons decay $v i a K^{-} \gg$ $\pi^{-}+\pi^{0}$ and are long lived. Because of this, only a few decay upstream of our photon detector. The resultant kaon triggers thus occur at about the same rate as the cascade radiative decays. Decays of $\Sigma^{-}$of ten produce soft $\pi^{-}$s upstream of the second hyperon magnet. They tend to be swept out of the main beam, and were vetoed by scintillators placed adjacent to the beam. 
Additionally, any beam decay tends to produce lower energy charged particles which then diverge from the beam when passing through the second spectrometer. The remaining undecayed beam may thus be vetoed by placing a scintillator directly in the beam, at the farthest downstream point in p-center. Combining all of these vetoes removes all but anti( $\left.\Sigma^{+}\right)$decays (figure 10 ). This was our most important background. Using the charged particle kinematics to separate the signal from the background, the final signal to background in the missing mass plot is probably no more than 10 times worse than what is seen for the sigma radiative decays. Unfortunately, the anti $\left(\Sigma^{+}\right)$decay kinematics overlaps the $\boldsymbol{\Xi}^{-}$decay kinematics. Thus, there is a class of anti $\left(\Sigma^{+}\right)$decays directiy under the $\Xi^{-}$events. Figure 11 shows the missing neutral mass for the decay $\Xi^{-} \gg \Sigma^{-}+$ $x$, with no cuts. The peak on the right represents undecayed beam tracks, and the smooth background is due to Anti $\left(\Sigma^{+}\right)$ciecays. After applying all of the cuts that were used in the $\Sigma^{-} \gg p+\gamma$ arialysis, one still cannot see a clear peak. To extract this signal on-line, an additional constraint was needed. This constraint turned out to be the lifetime difference between anti $\left(\Sigma^{+}\right) s$, and $\Xi^{-} s$. The lifetime of the these two particles is different by nearly a factor of 2 . Thus, the ratio of cascades to anti $\left(\Sigma^{+}\right) s$ changes as a function of $z$ position in the decay volume. By dividing the decay volume into two approximately equal sample sizes (for the anti( $\left.\Sigma^{+}\right)$events), and then subtracting the missing mass spectra thus obtained, the anti $\left(\Sigma^{+}\right)$s should vanish to first order, leaving only cascade decays. This was done, and a clear peak remains at zero-mass (figure 12). The width of the peak is roughly that which is expected from the Monte Carlo 
calculations. The next step in this analysis is to estimate the number of signal events in the peak, and the number lost in the subtraction. The peak can then be renormalized and subtracted from the original full spectrum data, and the signal to background and rough branching ratios thus estimated. This work is presently in progress and I should complete it in a couple of weeks. More sophisticated off-line analyses which will take into account the acceptances of the decays in question, and treat the spectrum as a continuum, rather than in 2 pieces, are being formulated.

\section{Additional Polarization and Magnetic Moment Results}

One of the more interesting results that appeared in 1989 was the E756 measurement of the Anti(cascade) polarization and magnetic moment. This was completely surprising in view of the prevailing idea (not theoryl) that the valence quarks were the major contributors to hyperon polarization. since there are no valence quarks in the anti $\left(\Xi^{-}\right)$, the polarization was expected to be small, as it is for anti( $\Lambda)$ s, and omegas. The measured polarization was about 10\%. Thus a lot of excitement was generated within the group to try to measure the anti $\left(\Sigma^{+}\right)$polarization and magnetic moment. To measure this, one simply switches both the hyperon magnet to produce negative beam, and the spectrometer magnets to analyze negative particles. Approximately $140 \mathrm{~K}$ anti $\left(\Sigma^{+}\right)$polarization events (with cuts) will be reconstructed. A final error in the polarization better than \pm 0.01 should be possible. For the magnetic moment measurement, it is necessary to produce the hyperon polarization perpendicular to the field of the hyperon magnet ( $B$ is vertical), so that it $w i l l$ then precess about the field. Near the end of the Sigma to $P$ gamma data acquisition period, we thus rotated the beam line magnets to allow us to 
produce the required horizontal polarization. We then took data with the hyperon magnet set for first positive and then negative beam. Using the positive beam, we measured the $\Sigma^{+}$ magnetic moment and with the negative beam, the anti $\left(\Sigma^{+}\right)$ magnetic moment (assuming the anti( $\left.\Sigma^{+}\right)$polarization is nonzero). The $\Sigma^{+}$magnetic moment measurement is interesting because of large disagreements between previous measurements (Table 5).

\section{TABLE 5}

\section{$\Sigma^{+}$Magnetic Moments}

$\begin{array}{lrll}2.479 \pm 0.012 \pm 0.022 & 137 k & \text { Wilkenson } & 1987 \\ 2.38 \pm 0.02 & 44 k & \text { Ankenbra } & 1983 \\ 2.30 \pm 0.14 & 14 k & \text { Settles } & 1979\end{array}$

On the basis of the data we have taken, and the results of a preliminary analysis by A. Morelos and P. Cooper, we will achieve an overall statistical error of \pm 0.0028 for the $\Sigma^{+}$ magnetic moment. This is somewhere between 3 and 9 times better than Wilkenson, et.al. depending upon how well we can handle our systematic errors.

For the anti $\left(\Sigma^{+}\right)$data, we expect to reconstruct about $34 \mathrm{k}$ "clean" events (Figure 11). When this is combined with approximately 130k of "normal" horizontal targeting (eg polarization) data, we should be able to do a reasonable job of measuring the anti( $\left.\Sigma^{+}\right)$magnetic moment.

One exciting goal of the group was to test the idea of using a silicon crystal to precess the polarization of a positively charged hyperon. This is done by using the channelling properties of a bent crystal. The lorentz transformed electric fields yield equivalent magnetic fields of 
the order of $10^{6}-10^{7}$ gauss (depending on the chosen bend angle), for the length $(5 \mathrm{~cm})$ of the slilcon crystal. This idea is worth testing, because of the possibllity of measuring the magnetic moments of short lived baryons (containing charm and/or beauty quarks). Approximately $5 \mathrm{k}$ of channeled $\Sigma^{+} \mathrm{s}$ were written to tape near the end of the run, and the analysis is currently underway. This amount of data should be sufficient to tell us whether this method is feasible. If it is feasible, we will then consider the impact on the next experiment that we will do in proton center, namely E781, which will produce copicus quantities of charmed baryons.

\section{Summary}

C. Newsom has spent approximately 11 of the last 15 months at Fermilab participating in experiment E761, hyperon radiative decays. During this period, all of the stated goals of the experiment were fulfilled or exceeded (tables 6 and 7 ). High precision data were obtained for all of the principal channels that were originally proposed. Also, we measured many additional quantities, including polarizations and magnetic moments of both $\Sigma^{+} s$ and anti $\left(\Sigma^{+}\right) s$. Finally, we succeeded in channelling $\Sigma^{+} s$ to test the idea of measuring magnetic moments via channelling in a silicon crystal. Final data analysis is now underway. 


\section{Table 6}

\section{The Proposed Goals of E761}

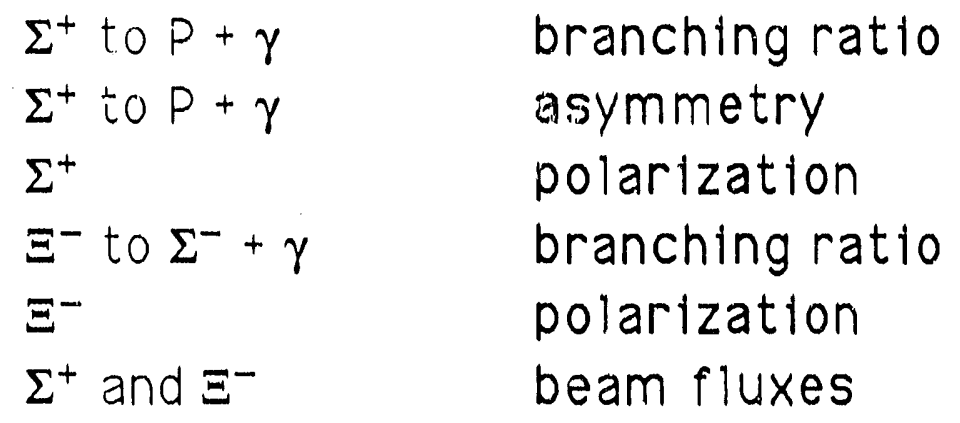

\section{Table 7}

\section{The Final Goals of E761}

$\begin{array}{ll}\Sigma^{+} \gg P+\gamma & \text { high precision branching ratio } \\ \Sigma^{+} \gg P+\gamma & \text { high precision asymmetry } \\ \Sigma^{+} & \text {polarization versus } p_{t} \\ \Sigma^{+} & \text {high precision magnetic moment } \\ \Sigma^{+} & \text {polarization via crystal channeling } \\ \text { anti }\left(\Sigma^{+}\right) & \text {polarization } \\ \Xi^{-} \gg \Sigma^{-+\gamma} & \text { branching ratio (asymmetry?) } \\ \Xi^{-} & \text {polarization } \\ \Sigma^{+} \text {and } \Xi^{-} & \text {beam fluxes versus } p_{t}\end{array}$




\section{Expenditure of Contract Funds}

During the period from 1 February 1990 to 30 september 1990, C. Newsom has spent approximately $80 \%$ of his budgeted portion of the contract account DE-ACO2-87ER40318 (figure 14). Although only $67 \%$ of the total budget perlod has passed, this is a reasonable balance due to the fact that the summer is a higher expense perlod. Based on an estimate using the previous non-summer months, the budget should be balanced to within a few percent by the end of the contract period. 
Eff Us DAC

Threshold (DAC/10) us CH \#
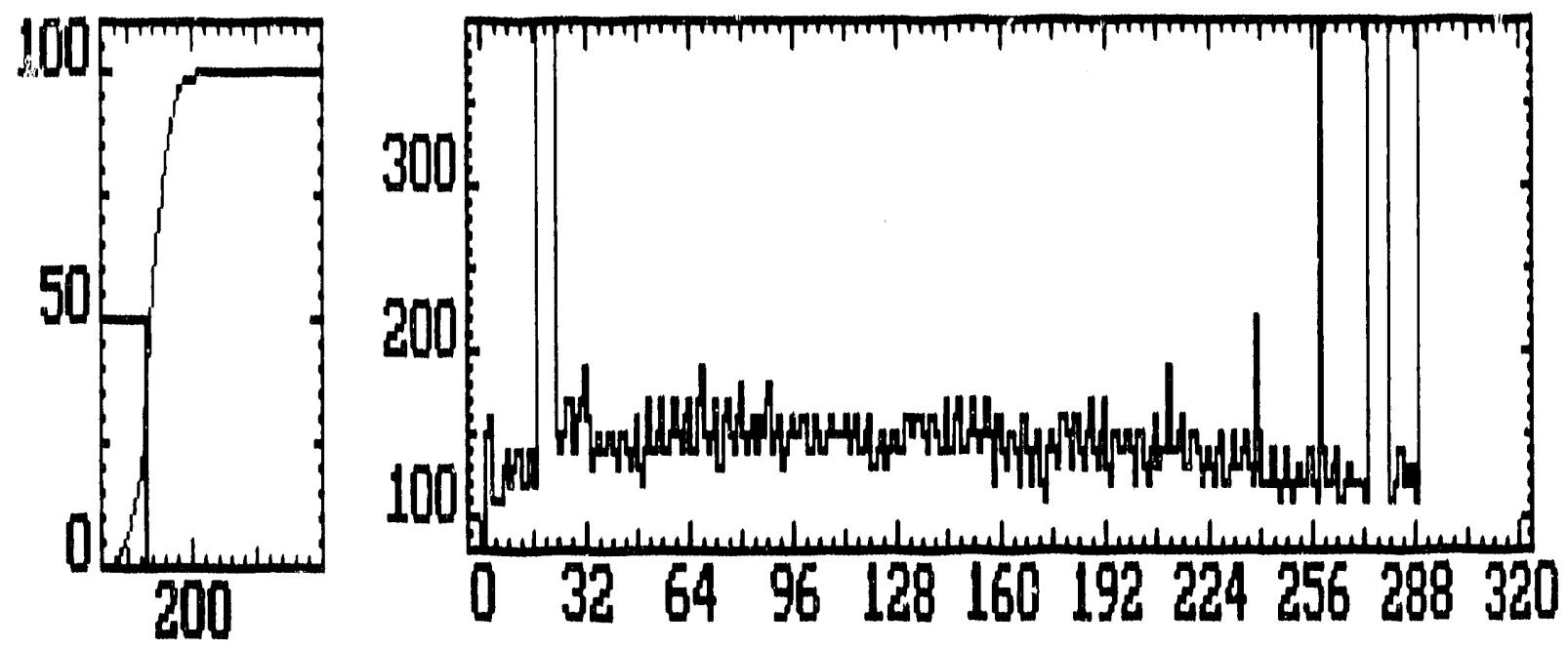

Eff us Del

50\% width (ns) vs CH \#
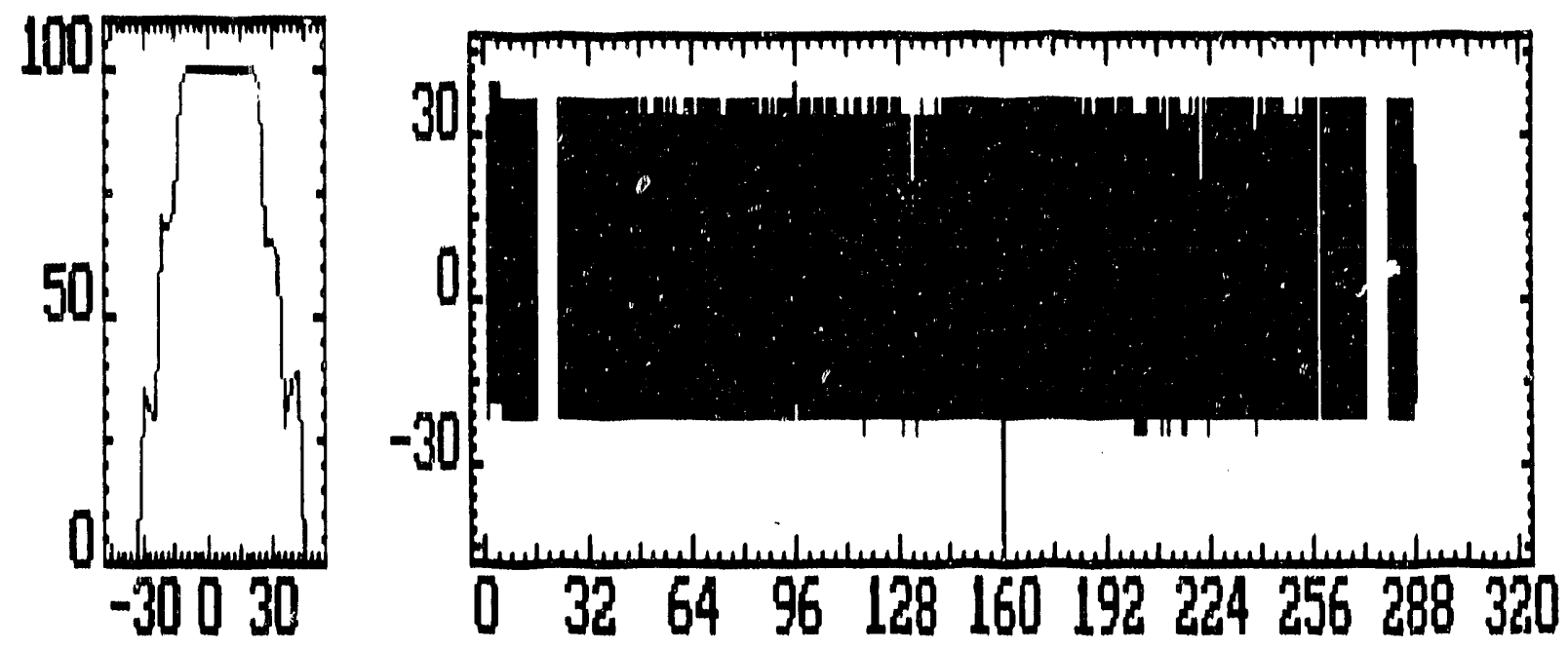

Figure 1. Threshold and de lay values for a sllicon plane using the IBM PC pulser system. Individual delay and threshold curves for one strip are shown to the left. 


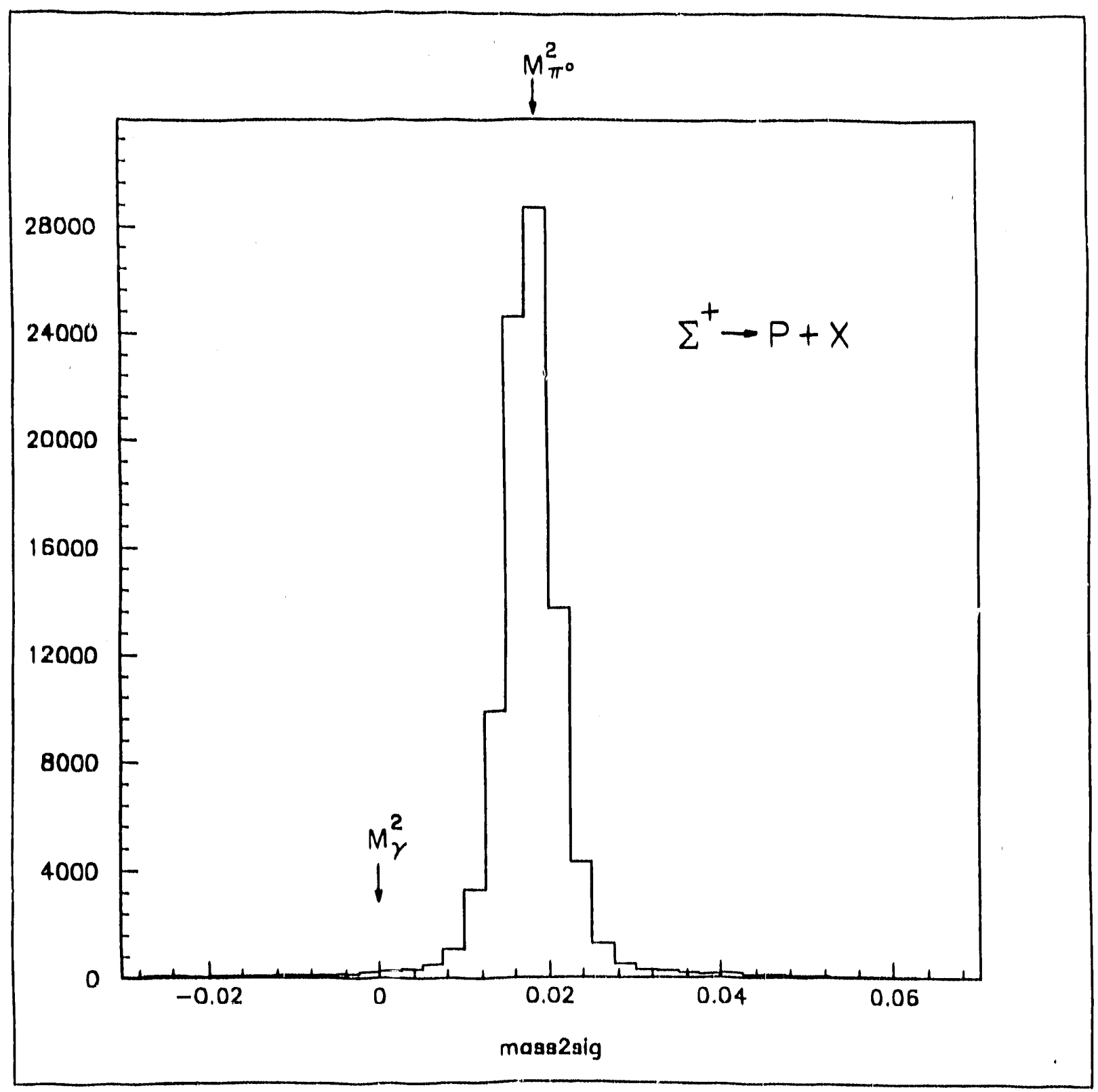

Figure 2. A histogram of the square of the neutral missing mass for the reaction $\Sigma^{+} \gg p+$ neutral with no cuts. 


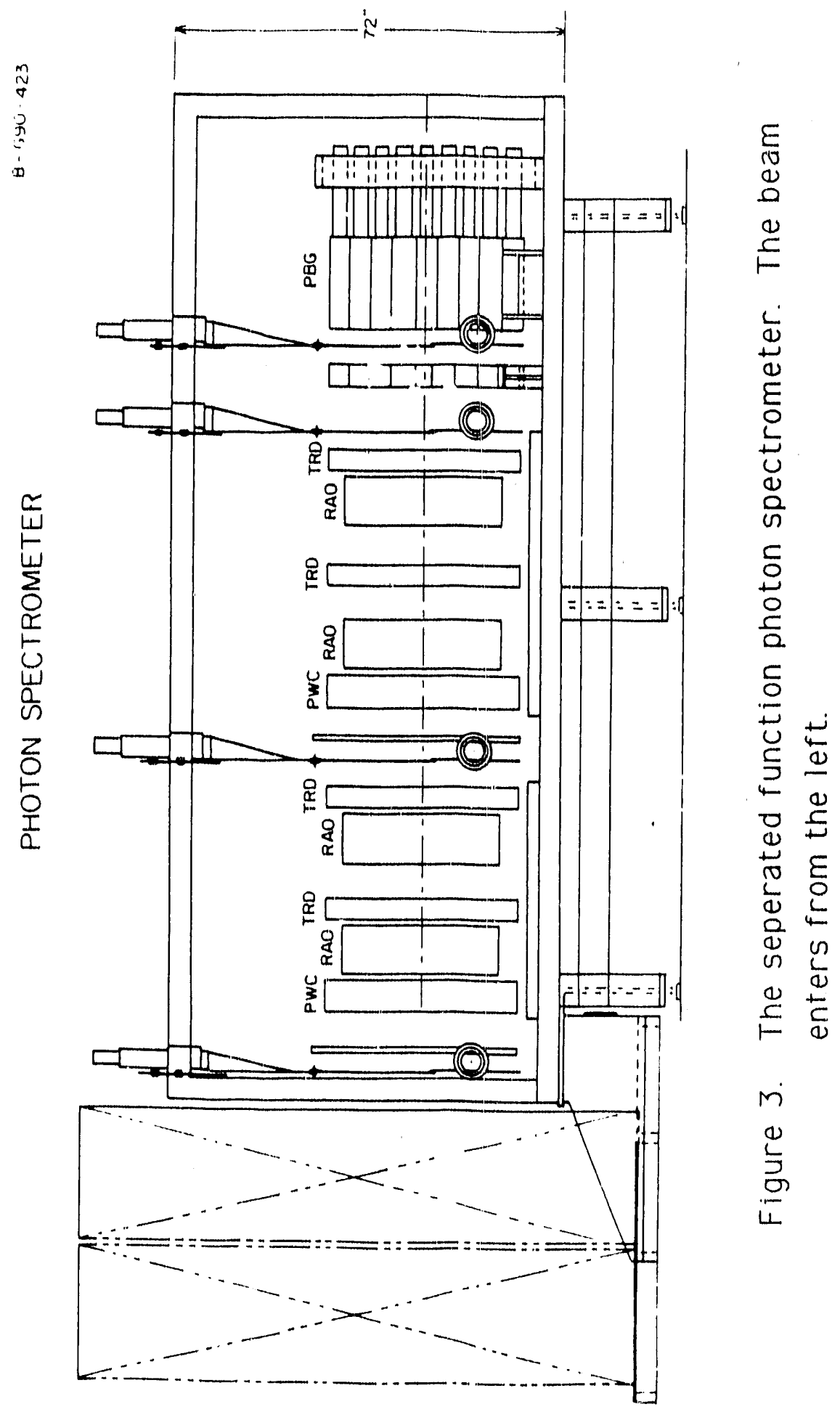




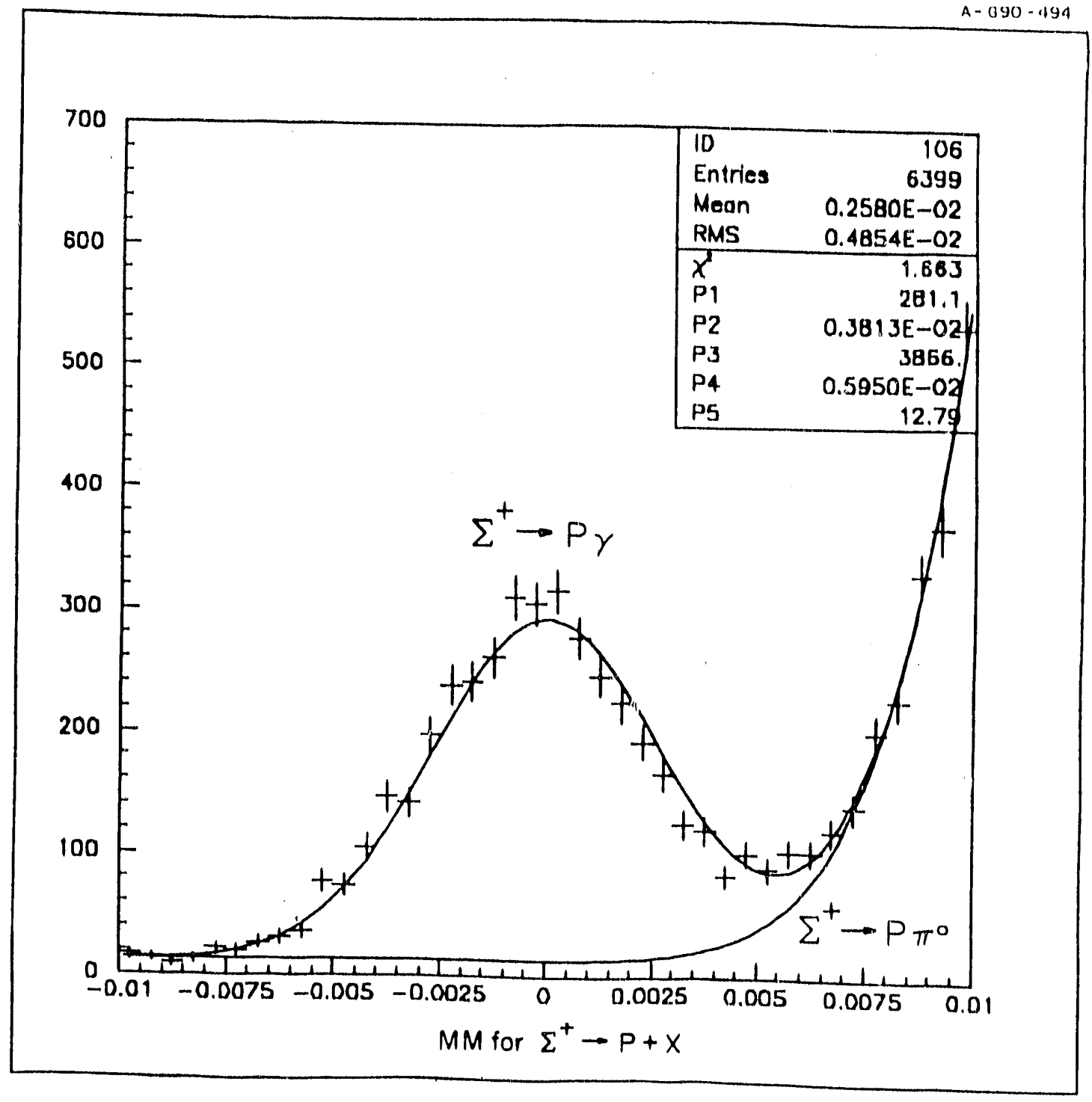

Figure 4. A histogram of the square of the neutral missing mass for the reaction $\Sigma^{+} \gg p+$ neutral with a photon required. 


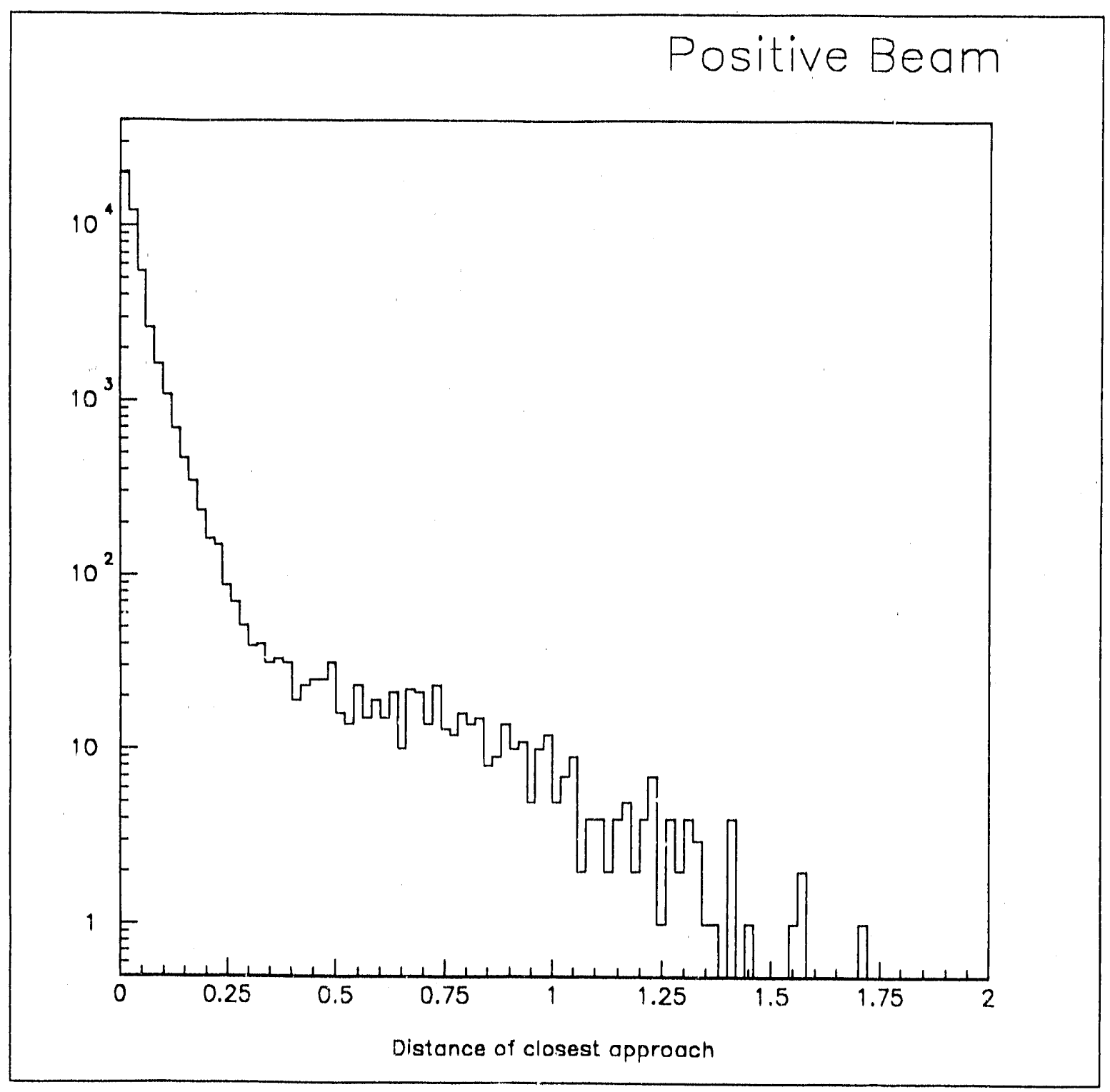

Figure 5. Distance of closest approach between the beam and the decay track. A clear break at $0.4 \mathrm{~cm}$ is seen. 


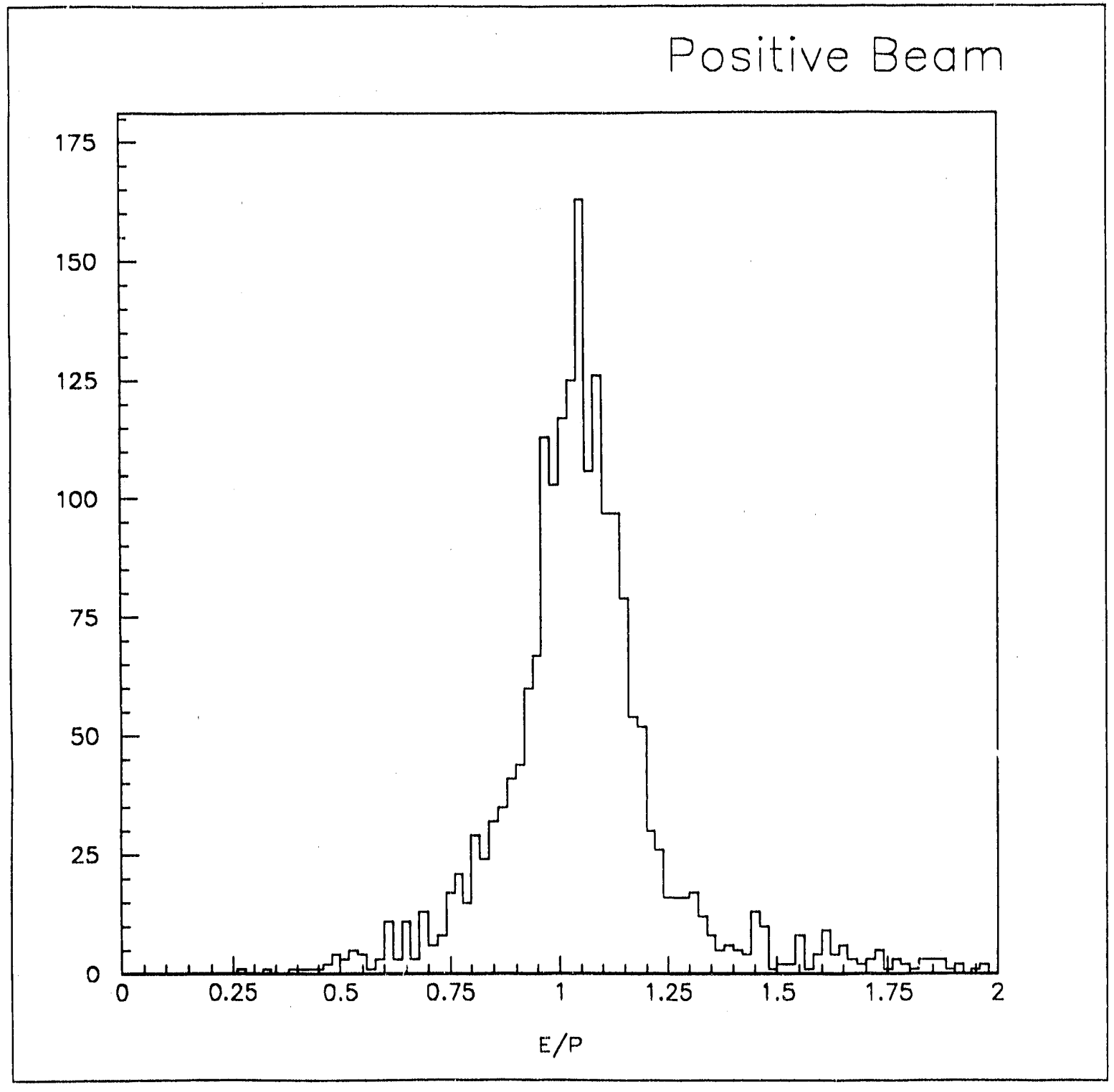

Figure 6. Energy / momentum for neutral particles. Massless particles and pi-zeroes should peak at 1.0. 


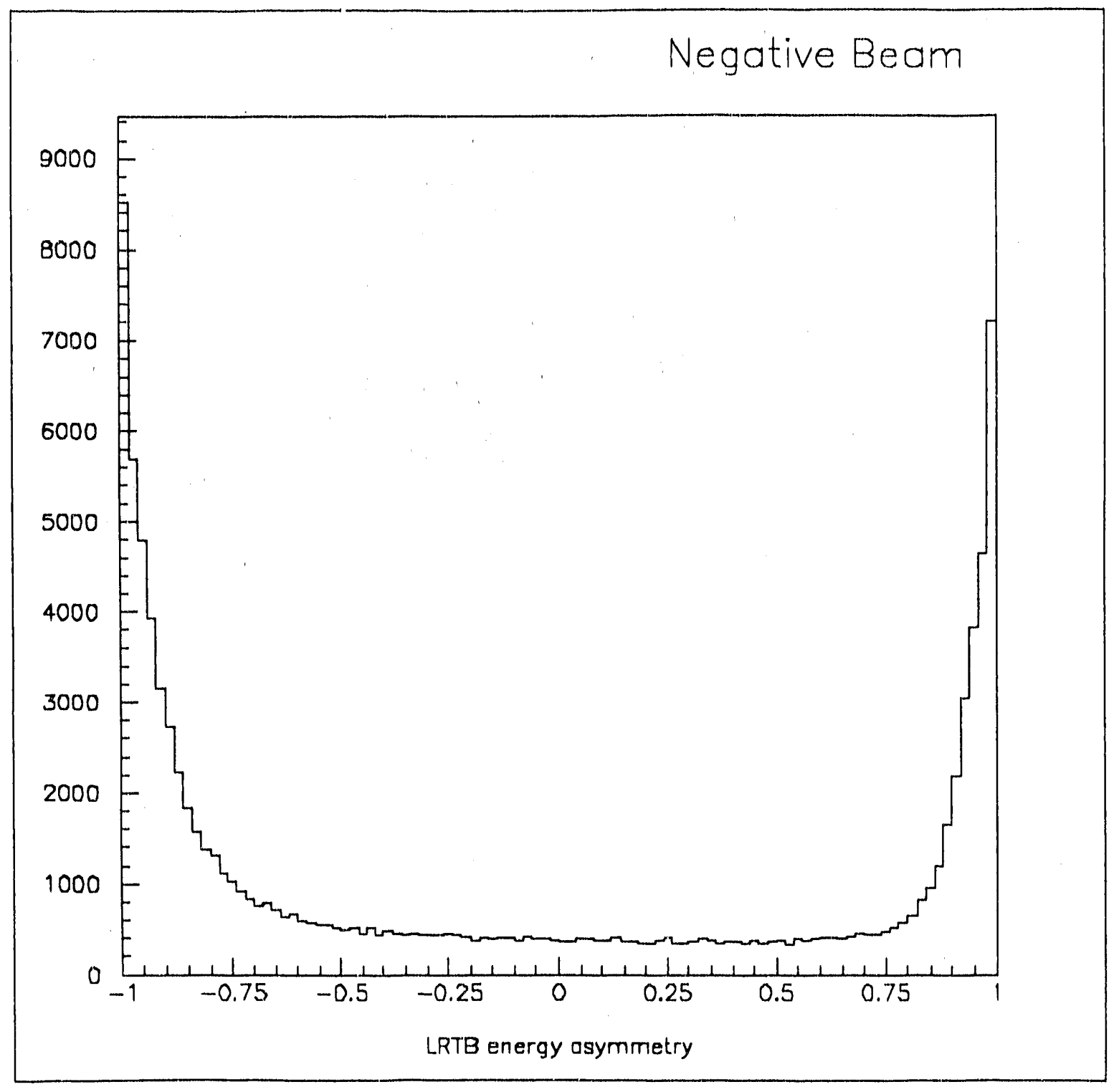

Figure 7. Left-Right and Top-Bottom energy asymmetry in the lead glass array. Events spread around zero are have 2 or more showers and thus may be cleanly seperated. 


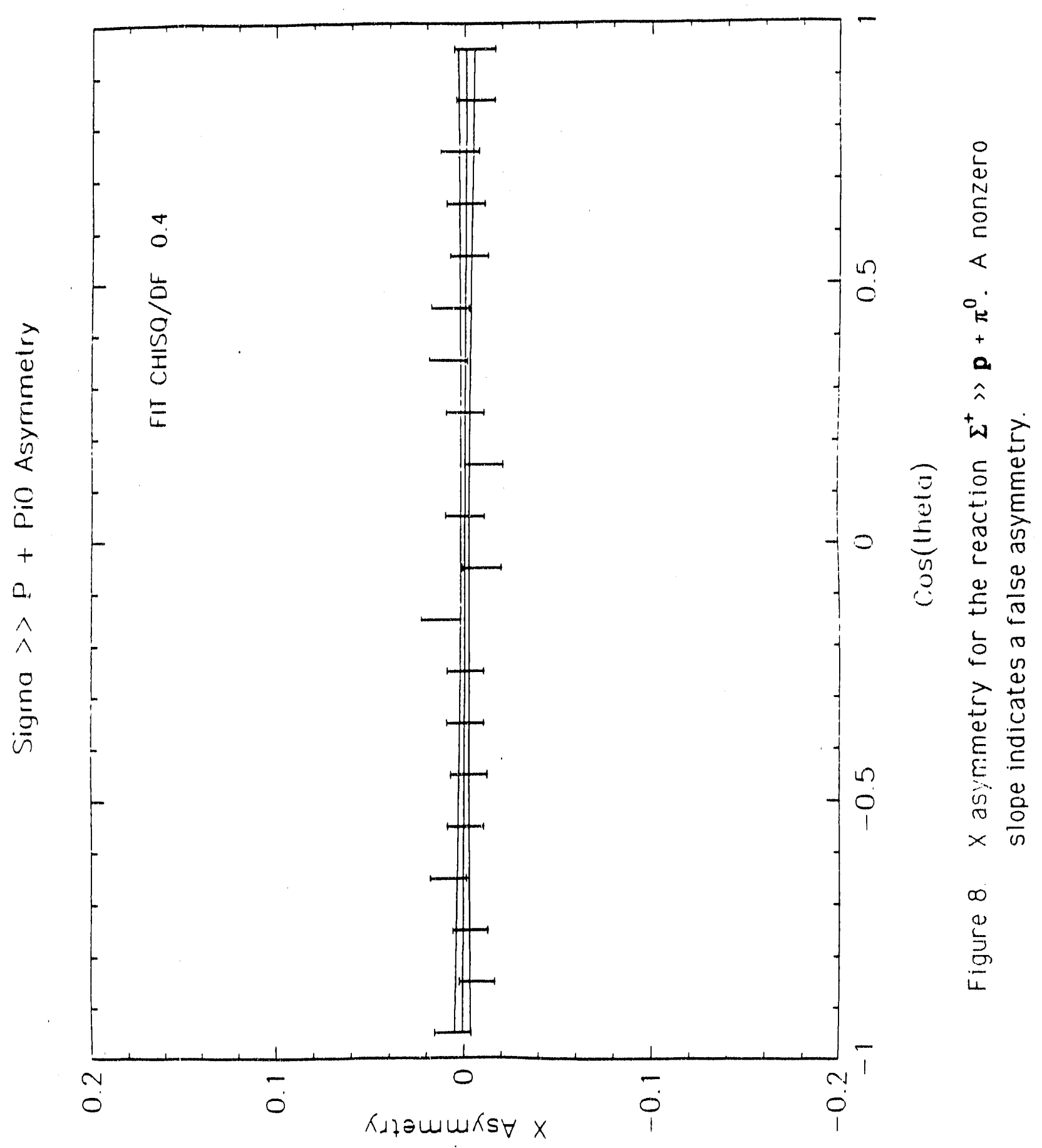




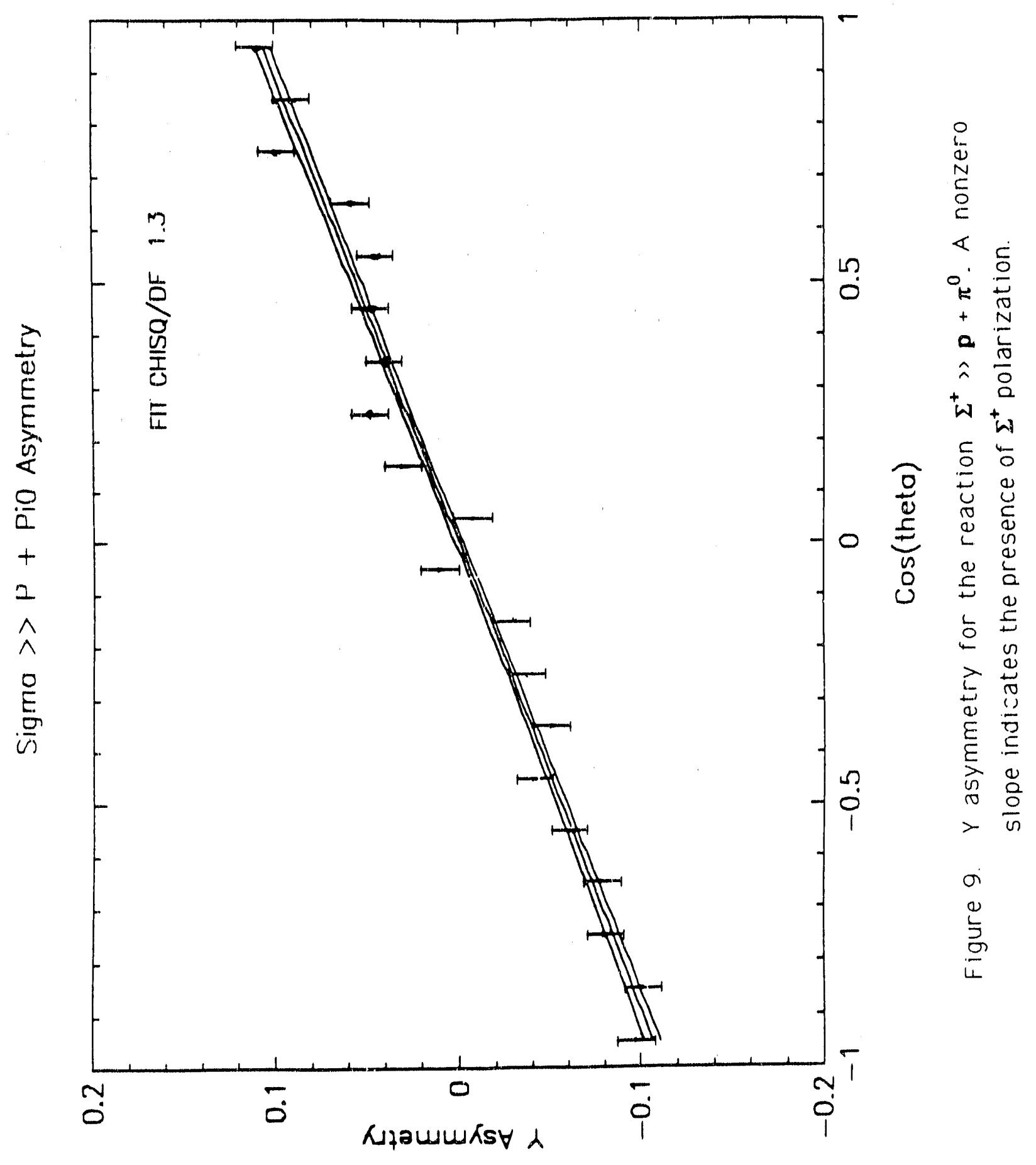




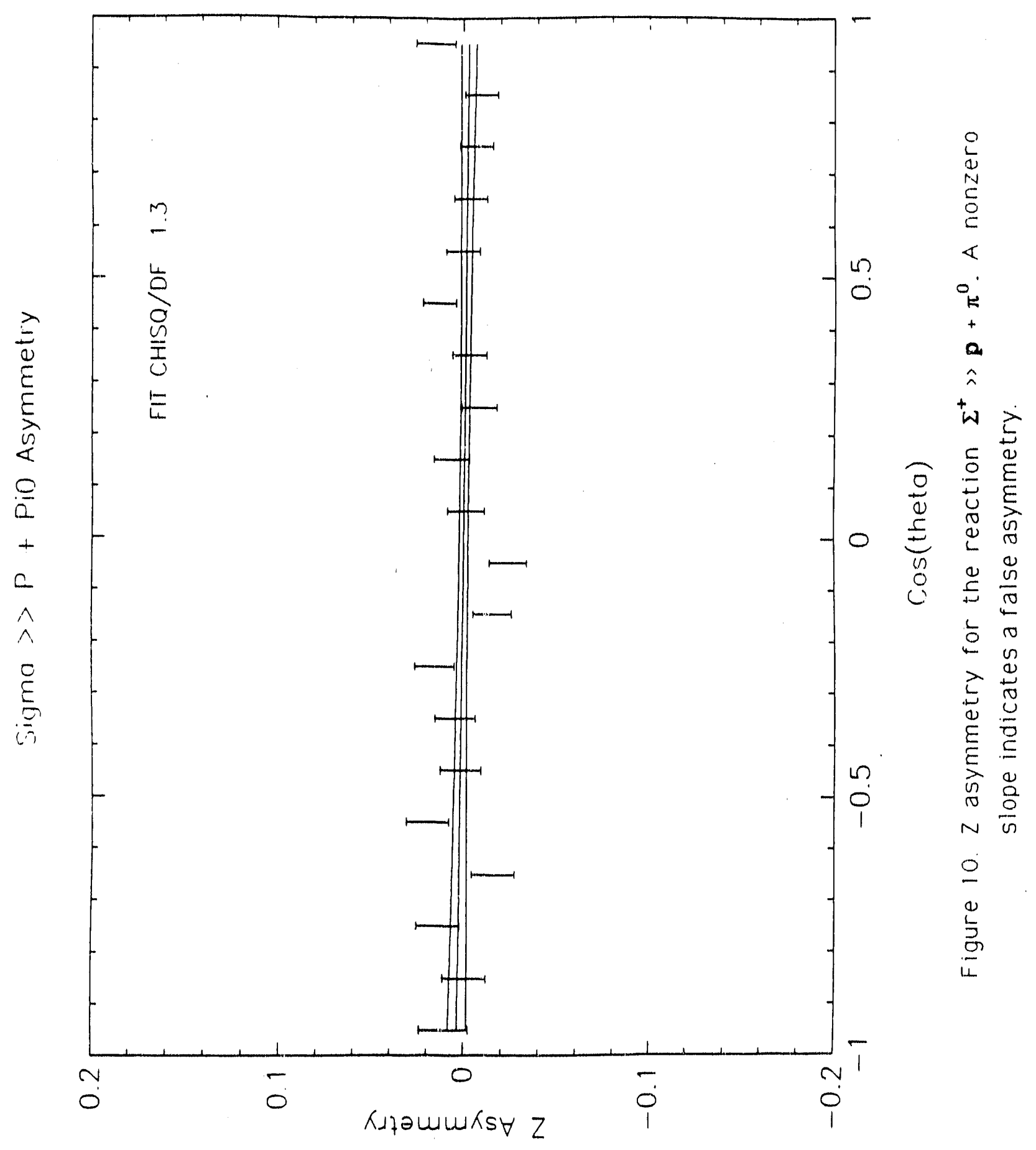




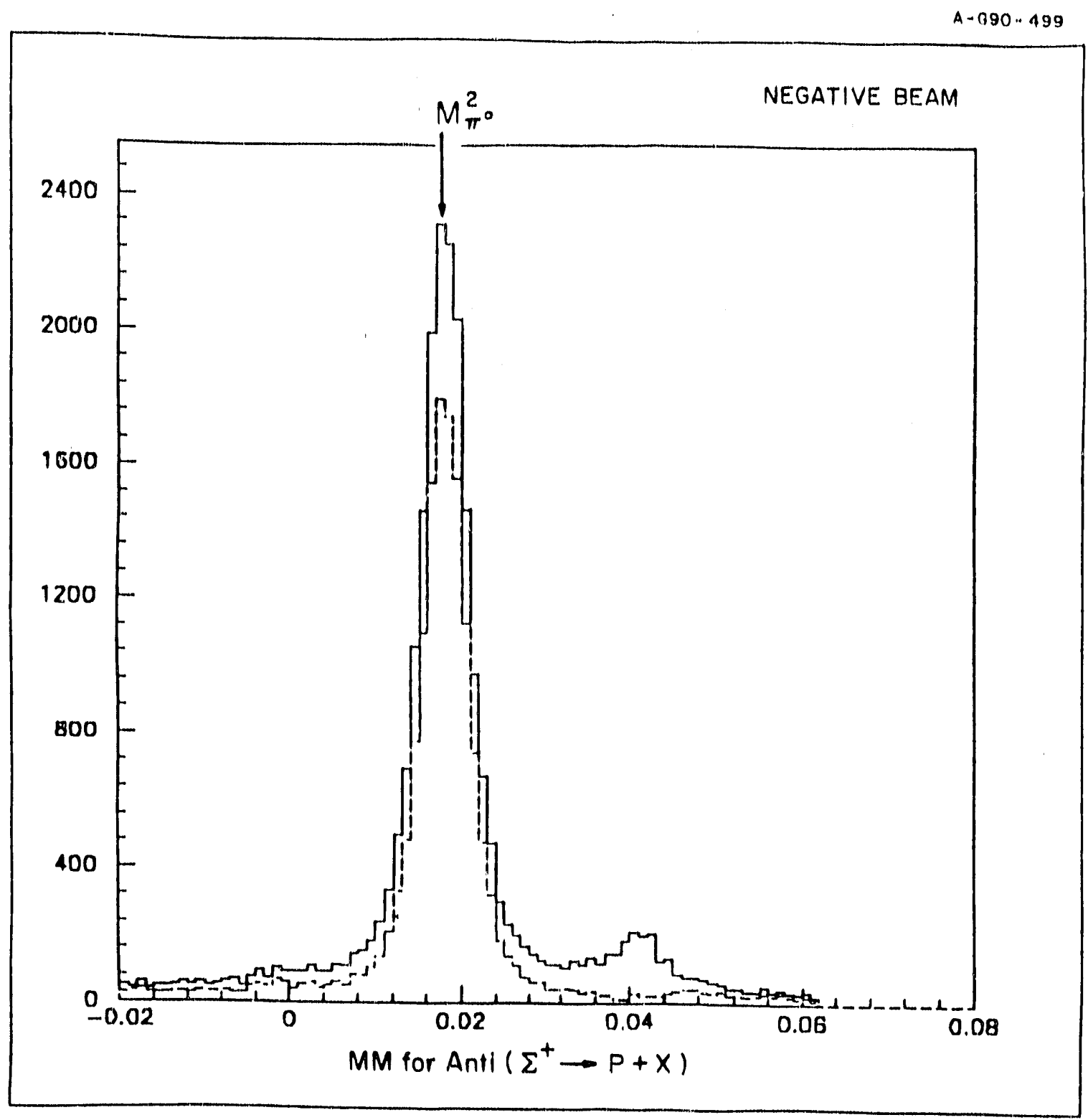

Figure 11. A histogram of the square of the neutral missing mass for the reaction anti $\left(\Sigma^{+} \gg p+\right.$ neutral $)$ with and without cuts. 


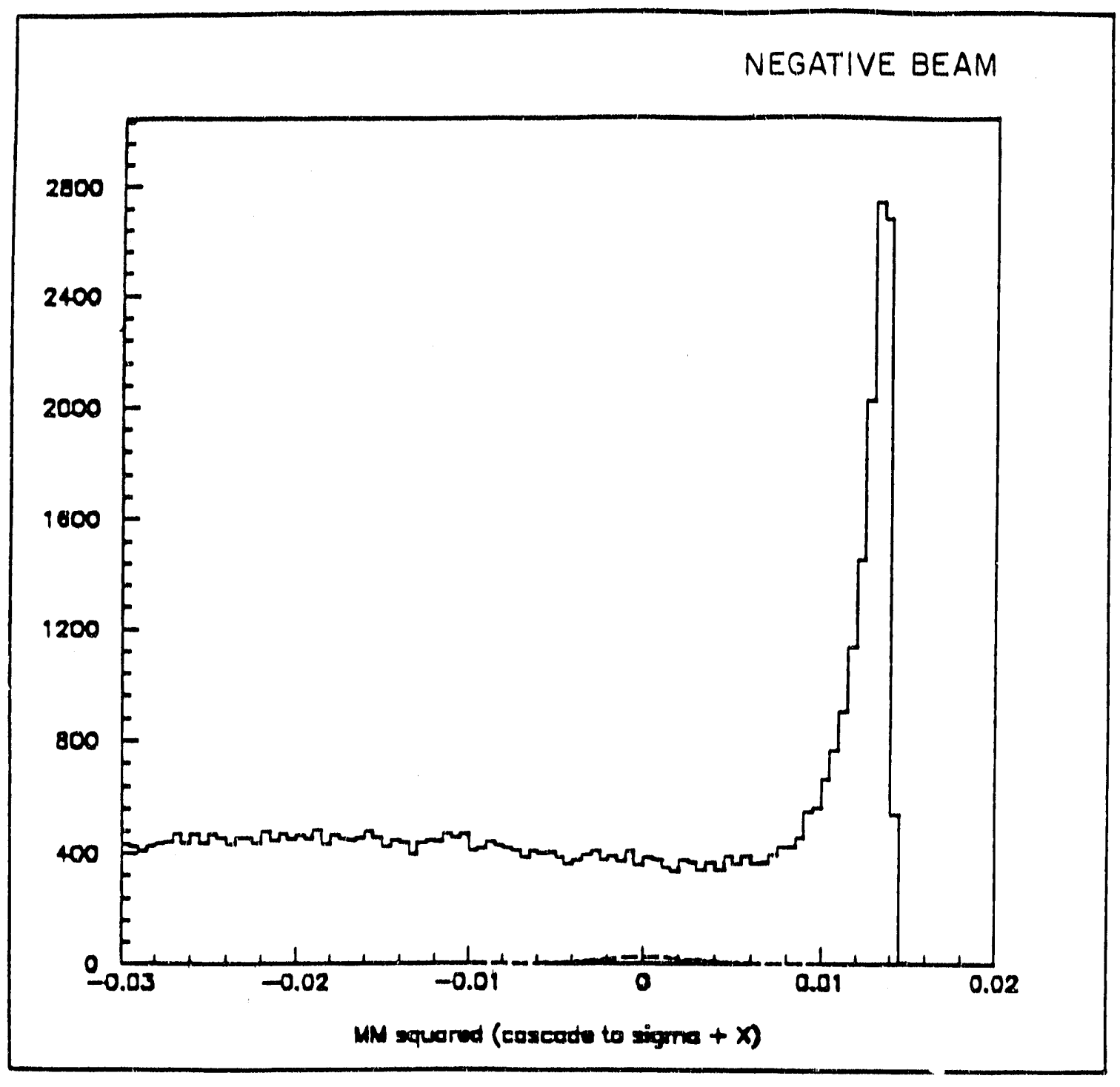

Figure 12. A histogram of the square of the neutral missing mass for the reaction $\mathbf{\Xi}^{-} \gg \Sigma^{-}+$neutral with no cuts. The small dotted line represents the approximate shape of the expected signal (arbitrary height). 


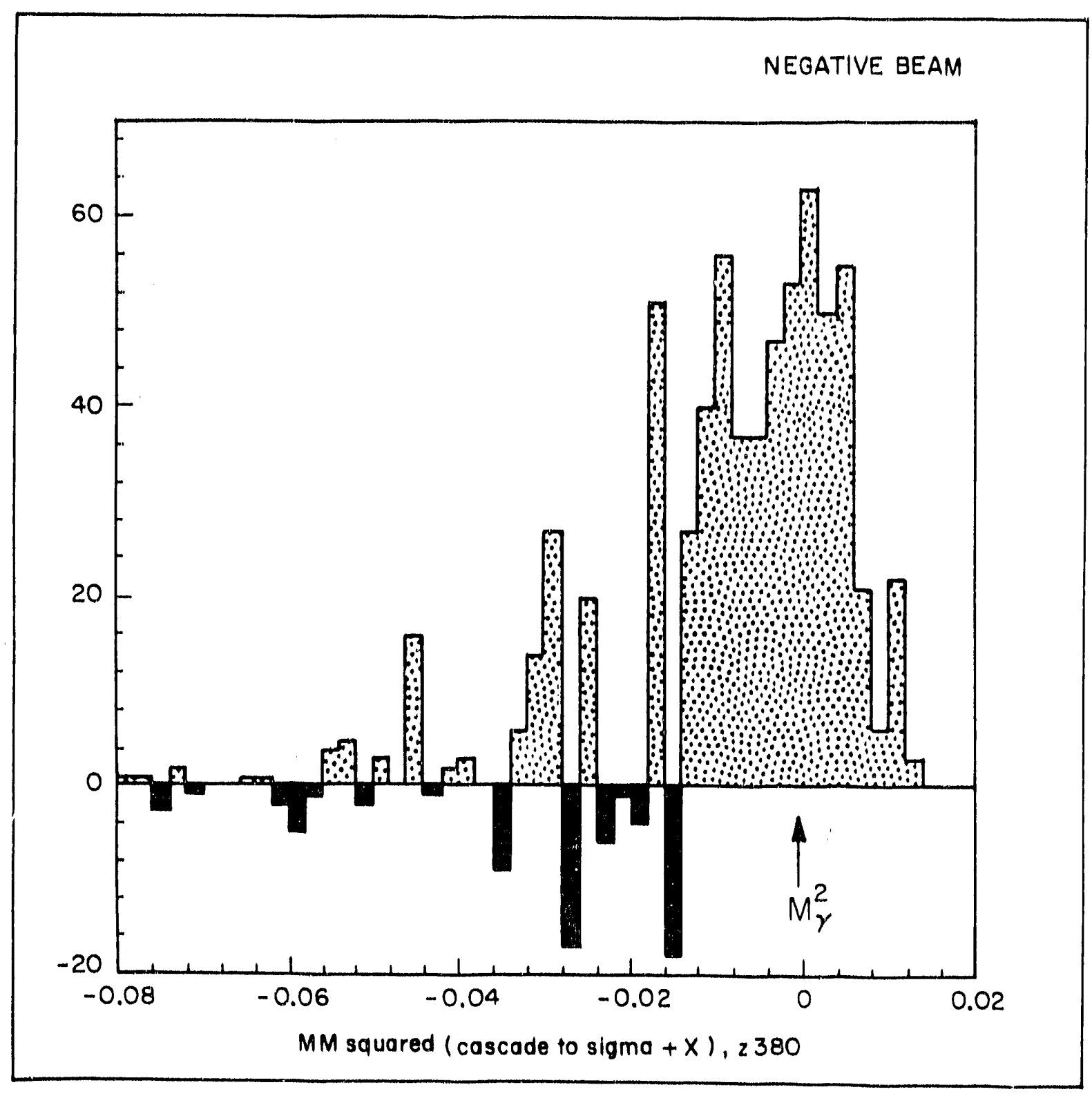

Figure 13. A histogram of the square of the neutral missing mass ior the reaction $\Xi^{-} \gg \Sigma^{-}+$neutral with all cuts. There is a clear peak at zero mass. The width roughly corresponds to the expected width of our signal, as indicated by Monte Carlo calculations. 


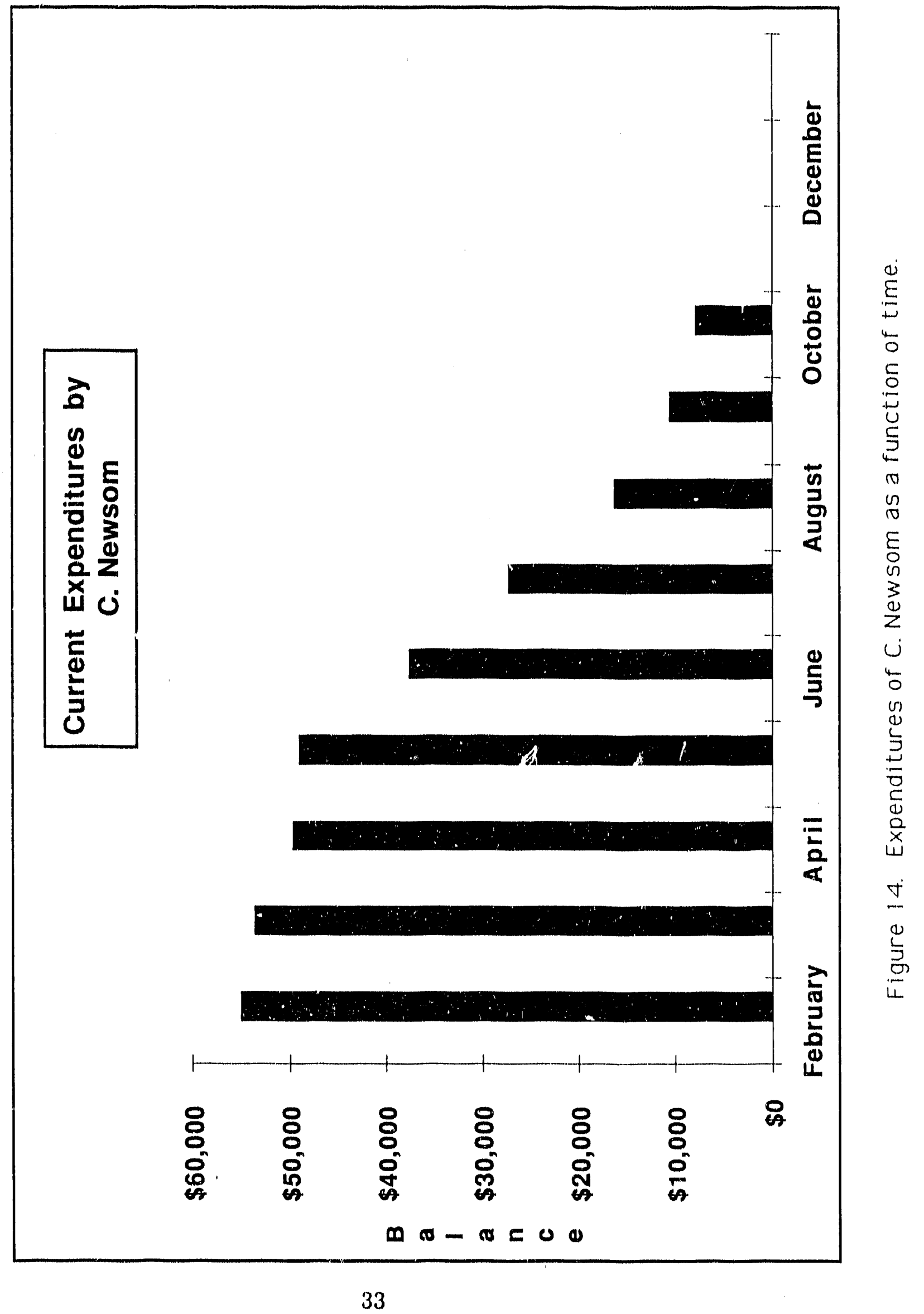




\section{PART B}

\section{Progress Report}

\section{Hadronic Interactions at Fermilab}

Y. Onel 


\section{Introduction}

The following report summarizes our work in high energy experimental physics between the months of September 1989 and September 1990. Our group consists of Yasar Onel, Associate Professor of Physics, Nural Akchurin, senior graduate student, Jerry Langland and Jim McPherson, graduate students, and Jane Nachtman, Presidential undergraduate scholar. As a part of our research efforts, the above members have spent three summer months (May 15 August 15) at Fermilab. Our research program principally involves the hadron physics experiments E-704 and E-683. It is a collaborative effort involving The University of Iowa and other research groups.

E-704 is the highest energy (200 GeV) spin physics experiment with incident polarized protons and antiprotons in MP9 Polarized Proton beamline. Since spin effects in higher energies have become one of the most serious challenges to QCD after the EMC data, this experiment focuses on the study of polarization effects in high energy pp scattering. E-683, on the other hand, investigates the photoproduction of high transvorse momentum jets at Wide Band Photon beamline. The main objective of this experiment is to measure the cross-sections of the QCD subprocesses, i.e., QCD Compton effect and quark-gluon fusion.

Our current commitments already include a year of running time at Fermilab, data analyses, improvement and mainienance of constructed detectors and two Ph.D., one M.S., and one undergraduate honors theses. We plan to improve our effectiveness at our Fermilab activities as well as to initiate a new research and development program for the next generation detectors at SSC.

The outline of our principal activities are listed below.

\section{$\underline{\mathrm{E}-704}$}

- CNI data analysis

- Study of the spin dependence of $\Lambda(\bar{\Lambda})$ with polarized $p(p)$ data analysis

- Rewiring and installation of the twelve planes of CERN-built MWPC"s. 


\section{$\underline{E-683}$}

- E-683 run (Dec. 1990 - June 1990) and maintenance of lowa built drift chambers

- E-683 analysis

\section{$\underline{\text { SSC }}$}

- We plan to initiate a research and development program on scintillating fibers for the new regime of the event detection and tracking in future multi-TeV collider experiments. This program will include real time digitization, techniques of the multihit topology, which would provide an essential back-up to the vertex tracking at future collider rates.

\section{E.704}

The E-704 program offers unique spin physics studies at high $\mathrm{p}_{\mathrm{t}}$ and large $\mathrm{xf}$ phenomena and also in the difference between total cross sections ( $p p$ and $p p$ ) in the pure helicity states at high energies.

During the present fixed-target run, we have performed the following measurements:

- CNI (Coulomb Nuclear Interference Measurements)

- Polarized-Proton (anti-proton) beam at $200 \mathrm{GeV} / \mathrm{c}$ on polarized target

- $\Delta \sigma_{\mathrm{L}}(\mathrm{p} \uparrow \mathrm{p} \uparrow)$ and $\Delta \sigma_{\mathrm{L}}(\mathrm{p} \uparrow \mathrm{p} \uparrow)$

- $\quad \mathrm{A}_{\mathrm{LL}}$ in $\mathrm{p} \uparrow \mathrm{p} \uparrow \rightarrow \pi^{0} \mathrm{X}$ at high $\mathrm{p}_{\mathrm{t}}$

- Polarized-proton beam at $200 \mathrm{GeV} / \mathrm{c}$ on hydrogen target

- $\quad \mathrm{p} \uparrow \mathrm{p} \rightarrow \pi^{\circ}$ at high $\mathrm{p}_{\mathrm{t}}$

- $\quad \mathrm{p} \uparrow \mathrm{p} \rightarrow \Lambda\left(\Sigma^{0}\right) \mathrm{X}$

- $\quad \mathrm{p} \uparrow \mathrm{p} \rightarrow\left(\pi^{ \pm}, \pi^{\mathrm{O}}\right) \mathrm{X}$ at large $\mathrm{xf}$

The primary activities of the Iowa group were the CNI run preparation, CNI run and analysis, and the study of spin dependence 
of $\Lambda$ with polarized $p$ beam. The CNI measurement is the Ph.D. thesis of Nural Akchurin.

\section{2.a. CNI}

Following are that have been studied and the others that are yet to be verified and/or studied in more detail.

First, we would like to give a summary of the entire CNI run. This fixed-target running period (1990), CNI group was allotted testing and data-taking periods at three different times. During the entire CNI running time we have accumulated 135 runs over 85 tapes.

Period I 10 March - 13 March (Test) Runs 1-16 (11 MT's)

Period II 13 May -15 May (Data) Runs 17-42 (18 MT's)

Period III 24 May - 3 June (Data) Runs $43-135$ (56 MT's).

\section{Period I:}

1. Global timing of trigger and check of logic is accomplished.

2. Chamber debugging and tuning take time.

3. Tuning of DAQ - many PDP crashes occur.

4. Target veto counters are tuned and their effectiveness is studied.

5. Timing of scalers for utility signals for $\mathrm{CNI} / \Delta \sigma \mathrm{L}$ is done.

6. Tuning of Pulse Shape Discrimination (PSD) of target signals.

7. No automatic Snake Magnet reversal takes place in this Period.

8. Straight track run is taken.

9. Live time fraction is $0.2 \%$.

\section{Period II:}

1. All downstream logic and timing are rechecked with antiproton beam.

2. Final Good Target (GT) signal and SUM Latch signals are in trigger.

3. Multistrip Detector (MSD) is installed.

4. SBF signal is introduced to trigger. 
5. After $\Delta \sigma_{\mathrm{L}}$ test runs, $\mathrm{CNI}$ comes back and runs straight tracks.

6. Problems with BM109 polarity found (wrong sign !).

7. Many PDP crashes occur due to bad memor/ location in the memory board.

8. Snake magnets now automatic but many software crashes take place and it is realized that the snake state was partial L-type !

9. Correlation between downstream trigger and target logic is poor.

10. BK chambers are in data-stream.

\section{Periosi III:}

1. Modifications to electronics are done to make it more compatible with $\Delta \sigma L$.

2. Target analog signals are timed to ADC's in the ugly duckling.

3. NW hodoscope introduced to overall trigger.

4. Initial stages of target electronics are retuned.

5. Major E704 Vax Cluster crash for $26 \mathrm{hrs}$.

6. Target thresholds are once more tuned.

7. Live time fraction is measured to be $27 \%$.

8. Problems with polarization signals from tagging electronics investigated.

9. Individual target PSD signals are retuned up to the balanced signal level.

10. Live time is measured to be $>50 \%$.

11. Snake Magnets are changed to N2 mode.

12. Gray Code limiter electronics gives problems.

13. Calibration runs are taken (Pedestals, straight tracks, etc.).

Phase III is when we took most of our useful data. $68 \%$ of the time (148 hours) our magnetic tape was mounted on the tape drive and most of that time (approx. 75\%) there was beam (approx. 110 hours). Typical beam intensities were at MP6SEM $9.8 \times 10^{11}$ and at MP9SC1 $1.7 \times 10^{7}$.

\section{Preliminary Studies and Results}

The first aim was to understand the global quality of data. From run 107 to 132 , on tape we had 1826988 raw events, on DST 466432 events and after the analysis we had total of 290234 events. From raw data to DST, $25.53 \%$ of the events survive. From DST, after the 
full analysis, $62.22 \%$ of the events remain usable. The table below shows the distribution of the events for all targets according to $|t|$ bins of $0.0005(\mathrm{GeV} / \mathrm{c})^{2}$ wide. The events that are selected have all the right selection criteria (up, down high percentage of polarization, good snake up and down, scattered right and left --up and down scattered particles are not included in the table) imposed on them. Of all the good events, $47.47 \%$ of them are good for asymmetry ( $\left.\mathrm{AN}_{\mathrm{N}}\right)$ calculations.

$\begin{array}{lll}\mathrm{t} \text {-bin } & \text { Average } \mathrm{t} & \text { Number of Events } \\ 1 & 0.0028 & 43073 \\ 2 . & 0.0072 & 31131 \\ 3 . & 0.0123 & 16527 \\ 4 . & 0.0174 & 12619 \\ 5 . & 0.0224 & 10924 \\ 6 . & 0.0274 & 9074 \\ 7 . & 0.0324 & 6661 \\ 8 . & 0.0373 & 4316 \\ 9 . & 0.0422 & 2212 \\ 10 . & 0.0493 & 1249\end{array}$

The numbers quoted above came from 20 tapes. We expect to analyze another 33 tapes (Runs 45 to 106), however, it is difficult to make an extrapolation about the number of good events based on the number events. Remaining tapes probably have lower good/total events ratio. If we were to make a guess, we will probably double the number of good events.

Following figures summarize the results of analysis thus far.

1. Figure 1.a-g For each target we calculate asymmetry as a function of the four momentum transfer squared. The solid line is the theoretical prediction by Kopeliovich and Lapidus. Some of the results are shown with the calculated errors. Data points that have low statistics are discarded. Last digit of ID number is the target number and ID $=257$ is the sum of all targets.

2. Figure 2.a-b Vertex construction of each target.

3. Figure 3.a-b ADC slow component vs $|t|$ for targets 3 and 6 .

4. Figure 4.a-b Linear and Gaussian fits to ADC distributions. 


\section{2.b. $\Lambda$ Measurements}

Our second main activity was in the study of $\mathrm{p} \uparrow \mathrm{p} \rightarrow\left(\mathrm{p}^{ \pm}, \Lambda, \Sigma^{0}\right)+\mathrm{X}$ reaction.

For the above measurement, we have installed three large lowa MWPC's ( 12 plaries) and took responsibility for the maintenance and running of the MWPC of forward spectrometer.

Fig. 5a-d show the online histograms for the Iowa Chamber efficiencies in the forward spectrometer. Fig. 6a-c show the reconstruction of $\Lambda$ peak using our forward spectrometer.

Data are currently being analyzed.

\section{2.c. Other Progress in E-704}

During the 1990 run, we have taken data on 3676250 b.p.i. tapes for the reaction $\mathrm{pp}(\mathrm{pp}) \rightarrow \pi^{0}+\mathrm{X}$ and this data is summarized as

- High $\mathrm{pt}_{t}$ single spin asymmetry $\mathrm{A}_{\mathrm{n}}$ at $\mathrm{x}_{\mathrm{f}}=0$.

Reactions:

$$
\begin{array}{ll}
\mathrm{p} \uparrow+\mathrm{p} \rightarrow \pi^{0}+\mathrm{X} & 1.0<\mathrm{p}_{\mathrm{t}}<5.0 \mathrm{GeV} / \mathrm{c} \\
\mathrm{p} \uparrow+\mathrm{p} \rightarrow \eta+\mathrm{X} & 2.3<\mathrm{p}_{\mathrm{t}}<4.0 \mathrm{GeV} / \mathrm{c} \\
\mathrm{p} \uparrow+\mathrm{p} \rightarrow \pi^{0}+\mathrm{X} & 1.0<\mathrm{p}_{\mathrm{t}}<4.0 \mathrm{GeV} / \mathrm{c}
\end{array}
$$

- Large $\mathrm{x}_{\mathrm{f}}$ single spin asymmetry $\mathrm{A}_{\mathrm{N}}$ for $0.2<\mathrm{p}_{\mathrm{t}}<2.0$ and $0.0<\mathrm{x}_{\mathrm{f}}<0.9 \mathrm{GeV} / \mathrm{c}$

Reactions:

$$
\begin{aligned}
& \mathrm{p} \uparrow+\mathrm{p} \rightarrow \pi^{0}+\mathrm{X} \\
& \mathrm{p} \uparrow+\mathrm{p} \rightarrow \pi^{0}+\mathrm{X}
\end{aligned}
$$

- Double spin asymmetry $\mathrm{ALL}_{\mathrm{L}}$ at $\mathrm{xf}=0$ and $1.0<\mathrm{p}_{\mathrm{t}}<3.0$ $\mathrm{GeV} / \mathrm{c}$. 
Reactions:

$$
\begin{aligned}
& \mathrm{p} \uparrow+\mathrm{p} \rightarrow \pi^{\mathrm{O}}+\mathrm{X} \\
& \mathrm{p} \uparrow+\mathrm{p} \rightarrow \pi^{\mathrm{o}}+\mathrm{X}
\end{aligned}
$$

The preliminary results for this channel are shown in figures 7-13.

\section{High $p_{t}$ single spin asymmetry $A_{N}$ at $x_{f}=0$}

In the reaction (1):

- The asymmetry is compatible with zero within 1-3\% accuracy in the $\mathrm{p}_{t}$ range from 1 up to $3 \mathrm{GeV} / \mathrm{c}$. It may be positive and about $3 \%$ near $\mathrm{pt}_{\mathrm{t}}=1.5 \mathrm{GeV} / \mathrm{c}$; however, it needs to be studied further. We have 30 tapes with the data in this region, but have not analyzed them yet.

- The asymmetry is negative between 3.1 and $3.8 \mathrm{GeV} / \mathrm{c}$ and equalis to $(38 \pm 10) \%$ in this region, that is about 4 standard deviations from zero.

- The data of BNL, CERN, Serpukhov, and E-704 are consistent. The sign is positive for polarized beam at BNL and FNAL, and negative for polarized target at CERN and Serpukhov. It shows a scaling $x_{t}$ behavior of the asymmetry in inclusive pion $\left(\pi^{+}\right.$and $\left.\pi^{\circ}\right)$ production.

- $\mathrm{x}_{\mathrm{t}}=0.4$ is a crucial point for asymmetry, and in this region asymmetry starts to increase. The maximal observed asymmetry values are of $25-35 \%$ and do not depend on energy in a wide energy range from 13 up to $200 \mathrm{GeV}$.

- There may be a general oscillatory behavior of single-spin asymmetry as a function of transverse momentum regardless of the reaction. Our data also indicate this. Earlier this behavior was clearly seen in the pp-scattering (BNL data), and in the quasi-elastic reaction $\pi^{-}+p \rightarrow \pi^{0}+n$ (Serpukhov data at $40 \mathrm{GeV} / \mathrm{c}$ ).

Although manifestations of this oscillation can be different; for example, $\mathrm{p}_{\mathrm{t}}$-dependence for elastic processes and $\mathrm{x}_{\mathrm{t}}$ dependence for inclusive production. The previous high-pt inclusive data (CERN, Serpukhov, BNL) do not contradict this hypothesis. 
- The asymmetries in the reactions (2) and (3) show no difference from the asymmetry in the reaction (1). There is an indication that they are negative in the region between 3 and 4 $\mathrm{GeV} / \mathrm{c}$. The asymmetry in the $\mathrm{h}$ inclusive production was also measured at Serpukhov and did not manifest any difference from the $\pi^{\circ}$ asymmetry either.

\section{Large $x f$ single spin asymmetry}

- The data show significant $\mathrm{x}_{\mathrm{f}}$ dependence of the asymmetry for both reactions (4) and (5). In the beam fragmentation region the asymmetry is positive, between 10 and $15 \%$ for protons, and about twice less for antiprotons.

- The average asymmetry in the reaction (4) is in very good agreement with the earlier E704 measurement at $185 \mathrm{GeV} / \mathrm{c}$ in 1988.

-The experiment has also determined with improved precision the $\mathrm{p} / \mathrm{p}$ ratios of the spin-averaged invariant cross-sections for inclusive $\pi^{\circ}$ production. The data show strong dependence on kinematics, with values comprised between 1 and 3 . The ratio is largest at small $\mathrm{pt}$ and large $\mathbf{x f}$.

\section{Double spin asymmetry all at $\mathrm{xf}=0$}

- The asymmetry ALL is small or zero for both the reactions (6) and (7) in the region $1<\mathrm{pt}_{t}<3 \mathrm{GeV} / \mathrm{c}$. If D. Siver's model is correct, gluons do not carry a significant part of the proton spin.

The results of 4 experiments:

\begin{tabular}{lllllc}
\hline Lab & $\begin{array}{l}\text { Energy } \\
(\mathrm{GeV} / \mathrm{c})\end{array}$ & Reaction & Mean $\mathrm{P}_{\mathrm{t}}$ & $\mathrm{X}_{\mathrm{t}}$ & $\begin{array}{c}\text { Asymmetry } \\
\%\end{array}$ \\
\hline BNL & $13-18$ & $\mathrm{p} \uparrow+\mathrm{p} \rightarrow \pi^{+}+\mathrm{X}$ & $1.7-2.0$ & 0.7 & $25-30$ \\
CERN 24 & $\mathrm{p}+\mathrm{p} \uparrow \rightarrow \pi^{0}+\mathrm{X}$ & 2.2 & 0.7 & 25 \\
IHEP 40 & $\pi^{-}+\mathrm{p} \uparrow \rightarrow \pi^{\circ}+\mathrm{X}$ & 2.5 & 0.6 & $36 \pm 7$ \\
E704 200 & $\mathrm{p} \uparrow+\mathrm{p} \rightarrow \pi^{\circ}+\mathrm{X}$ & 4.2 & 0.45 & $38 \pm 10$
\end{tabular}


One can reach a conclusion that pion asymmetry at $x_{t}$ greater than 0.4 (cross-point with $\mathrm{pt}_{\mathrm{t}}$ axis) rises up to $25-35 \%$ and does not depend on energy in the energy range from 13 to $200 \mathrm{GeV}$.

\section{Future "follow-up" experiments}

Our present collaboration has already started to discuss the following for the 1992 Fixed Target Run at FNAL.

1. The energy upgrade of the MP beam line to $500 \mathrm{GeV} / \mathrm{c}$.

2. Spin dependence in direct gamma production at high $\mathrm{p}_{\mathrm{t}}$.

3. Spin dependence of $\chi_{2}$ (3555) production simultaneously with (2).

4. Studies of the energy dependence of various reactions studied in E-704 such as $\Delta \sigma_{\mathrm{L}}(\mathrm{pp})$ and asymmetries in $\pi^{\circ}, \eta^{\circ}, \Lambda / \Sigma, \pi+$ production at large $p_{t}$ or large $x_{f}$.

5. We intend to study the possible increase of the quark-diquark content of the proton versus $p_{t}$ in $A_{N}\left(p \uparrow p \rightarrow\left(\pi^{0}, \pi^{+}, \pi^{-}\right)+x\right.$ at $\mathrm{p}_{\mathrm{t}}$ up to $6 \mathrm{GeV} / \mathrm{c}$ with $\Delta \mathrm{A}_{\mathrm{N}}<5 \%$. We intend to test PQDC predictions based on subprocesses such as $u u \rightarrow u u, u d \rightarrow u d$, $\mathrm{qg} \rightarrow \mathrm{qg}$ and also on the gluon spin distribution in $\mathrm{A}_{\mathrm{LL}}(\mathrm{p} \uparrow \mathrm{p} \uparrow \rightarrow$ $\pi^{\circ}\left(\right.$ also $\left.\left.\pi^{ \pm}\right)+\mathrm{x}\right)$ at $\mathrm{p}_{\mathrm{t}}$ up to $5 \mathrm{GeV} / \mathrm{c}$ with $\Delta_{\mathrm{LL}}<5 \%$.

6. A sensitive way of analyzing the gluon polarization inside a polarized proton will be to measure the hyperon production at large $\mathrm{p}_{\mathrm{t}}$ collisions. The dominant mechanism for associated strange-particle production at large $p_{t}$ is strange-quark production from gluon-gluon annihilation. Theoretical predictions suggest absolute values of about $20 \%$. This measurement, using the E-704 facility, only becomes feasible at $500 \mathrm{GeV} / \mathrm{c}$ and is not feasible at $200 \mathrm{GeV} / \mathrm{c}$ because of the geometrical acceptance.

$A_{L L}\left(p \uparrow p \uparrow \rightarrow \Lambda^{0}+x\right)$

high $p_{\perp}$ up to $3 \mathrm{GeV} / \mathrm{c}$ with error $=5 \%$.

$D_{L L}\left(p \uparrow p \uparrow \rightarrow \Lambda^{\circ} \uparrow+x\right)$

7. Finally, we are considering the possibility of studying asymmetry in jet production (at present, we do not have a jet detector in the E-581/704 set-up). This process at high $\mathrm{p} \tau$ is dominated by gluon-gluon or quark-gluon scattering. 
Although this process is not as clean as the prompt photon production in the determination of the glnon-spin distributions, the cross section is about an order of three higher than that of the prompt photon which is therefore sulted for higher $p_{\perp}$ coverage.

$$
\begin{aligned}
& A_{N}(p \uparrow p \rightarrow \text { jet }+x) \\
& A_{L L}(p \uparrow p \uparrow \rightarrow \text { jet }+x) \quad \text { high } p_{\perp} \text { coverage, } p_{\perp}>6 \mathrm{GeV} / \mathrm{c}
\end{aligned}
$$

The follow-up of the E-704 program at UNK, U.S.S.R. (proposal Polex) We are currently discussing a possible cooperation on the POLEX experiment at UNK.

\section{3. $E-683$}

The past year has seen the completion of installation of the E-683 apparatus. The wire chambers, both upstream and downstream of the magnet were installed during the summer of 1989 . The beam calorimeter was completed and preliminary tests with a source were carried out during the fall of 1989. The muon hodoscope was installed during the summer of 1989. The experiment passed all safety inspections in February of 1990.

Beam was first delivered to Wide Band in February of 1990 . The experiment upstream of E-683 (E-687) began data-taking, while we began parasitic studies of both calorimeters and wire chambers. This period of parasitic studies allowed us to balance the calorimeter phototube high voltages, as well as bring all wire chambers to operating condition. By May of 1990 both the main calorimeter and beam calorimeter had been checked out and all the phototubes balanced. Time distributions for all drift chambers had been obtained, and the relevant parameters (drift velocities and zero times) had been obtained.

During the first week of June 1990, E-683 received a one week engineering run. Our goals for this test were:

1. Establish a beam tune and spot size at our target

2. Test of our triggering and readout of upstream $\mathrm{Si}$ chambers which are part of the beam tagging 
3. Preliminary calibration of both calorimeters and rate studies on the beam calorimeter

4. Preliminary high pt data

5. Tests of the effect of E-687 material in the beam, as a test of possible simultaneous running of two experiments.

The beam tune was achieved with no trouble, and the spot size was as calculated from beam transport programs.

Since the Wide Band beam has a momentum spread of $\pm 15 \%$, the upstream tagging information, which tags the momentuin of the incident beam to $2 \%$, is necessary both for calibration and photon tagging. Due to problems with software (provided by Italiar. collaborators on E-687), we were not able to read out the upstream tagging chambers until $11 / 2$ days into the test run. After the initial problems were solved, the beam tagging worked well. Figure 14 shows a typical beam energy distribution as measured by the silicon chambers.

Since the beam calorimeter was the first detector we studied, most of the calibration data for it was taken without the beam tagging information. Nevertheless, we did establish that the detector is working, and rate studies indicate that it will withstand expected counting rates.

Calibration data at both $60 \mathrm{GeV}$ and $150 \mathrm{GeV}$, and with both pions and electrons, was taken for the main calorimeter at several locations. A small amount of calibration data which was taken with the tagging working was also taken for the beam calorimeter.

One concern we have (this applies to both the main calorimeter and beam calorimeter) is the width of the distributions once the beam energy is divided out. Both calorimeters show a width about $50 \%$ wider than expected. We are currently studying possible explanations, including drifts in magnet currents or improperly balanced phototubes.

The high pt data immediately pointed to a problem with the muon veto wall. About half of the triggers were muons. These art: fairly easily eliminated off-line, but nevertheless this ratc is unacceptably high. After the test run was over, we discovered that many of the scintillator glue joints were both broken and yellowed. Some repairs 
were done right away, but the hodoscope will receive a reworking during the three-month accelerator down time.

In spite of the muon triggers, we did acquire a modest amount of high pt data, mostly taken with a "global" or large solid angle trigger.

Our hydrogen target was not operational for this test, so we took data with lead and beryllium targets. Comparison with hadron data from E-609 is interesting. The planarity distribution (Figure 15) from photon-induced events is nearly identical to that obtained from a hadron beam. However, we do not yet know how this distribution changes with $E_{t}$ cut. It shifts only slightly from lead to beryllium. Figure 15 is data taken with a lead target.

Figure 16a shows the distribution of energy in the beam calorimeter for photon-induced high $\mathrm{pt}_{\mathrm{t}}$ events and Figure $16 \mathrm{~b}$ shows the same distribution for hadron-induced events. The difference her is striking. As expected, little energy goes in the forward direction for photon-induced events compared to hadron-induced events.

The track reconstruction program using wire chamber information is in a preliminary working state. Rough vertices have been reconstructed, work is continuing.

The studies of beam conditions with upstream material are still underway, but so far no serious incompatibilities have been discovered.

During the week of August 20-27 E.683 will have a second week of test beam. During this time we will concentrate on preliminary high $\mathrm{pt}$ data with lead and beryllium targets. It is unlikely that our hydrogen target will be ready for this run.

\section{3.a. Progress on Iowa Drift Chambers}

The Iowa chambers consist of five drift chambers and one delay line chamber. These measure $2.5 \mathrm{~m}$ by $1.5 \mathrm{~m}$ each, with two chambers housed in each gas-tight box, making a total of three boxes. As of August 1989, preliminary testing had been completed at Lab 6 . The three chamber boxes had been moved to Wide-Band Photon Lab and 
installed on a roller support system designed for easy access to the chambers, should the need arise during the upcoming run. Temporary gas lines and high voltage supplies were also installed at that time.

In the past year, several objectives have been accomplished. all amplifier cards have been tested and installed on the chambers, along with their respective ECL ribbon cables and low voltage power. Final installation of HV and signal cables, TDC and trigger electronios, and gas supply system have been completed and tested.

A precise survey of the chambers on the beam-line was completed in November 1989, and a mapping of wire position to TDC channel no. was undertaken at that time. Since then, data acquisition software has been developed on the E-683 microvax to provide time distributions, hitmaps, and event tracking (see Figures 17a-e).

Thus far, we have successfully tested all six chambers in $80 \% \mathrm{Ar} /$ $20 \% \mathrm{CO}_{2}$, and in the higher-gain mixture of $50 \% \mathrm{Ar} / 50 \%$ Ethane with 0.1 deg $C$ isopropanol. E-683 has had two one-week engineering runs thus far this summer, during which the lowa chambers saw actual bearn. The chambers are now fully operational and ready for the upcoming December startup date.

Future work on Iowa chamber systems include:

1. Calculation of the effect of magnetic field on drift times.

2. Continuation of the gas studies performed on these chambers.

3. Analysis of the data taken under various conditions and gas mixiures.

4. A precise study of alcohol additives.

These last three objectives are a natural extension of our ongoing work on radiation damage to wire chambers. it is of interest to us to pursue this work further. We hope that we can shed clearer light on these phenomena by putting our early ideas to more rigorous tests in the future. As SSC physics, for example, will require reliable, high-rate and efficient detectors, we feel that it is very important to continue this study. It is unrealistic to expect that our current detector technology will be capable of handling tomorrow's vast challenges. 


\section{Conclusions}

The present task of Yasar Onel (Hadronic Interactions at Fermilab) started in November 1986 at the University of Texas at Austin. The progress of the group of hadronic interactions can be summarized as:

1. E-704

- running and analysis of CNI measurements (test run 1988)

- improvements on new CNI measurements

- new trigger

- new LED system

- on-line system using Iowa Vaxstation 3200

- CNI measurements (run period 1990)

- CNI measurements analysis

- installation of 3 large MWPC Iowa in the forward spectrometer

- $p \uparrow p \rightarrow \Lambda\left(\Sigma^{0}\right) X$ run is completed

- Data analysis of above has started

2. E-683

- construction of 6 drift chambers

- construction of a permanent test facility for wire chambers at Lab 6, Fermilab

- chambers installed in the Wide Band and fully operational

- engineering run or E-683 
We have thus far managed through the commitments and financial support of the University of Iowa. E-683 will come on-line between December 1990-June 1991. Nural Akchurin took his Ph. D. data on E704. Jim McPherson will be working toward his Ph. D. on E-683. Jerry Langland and Jane Nachtman will be working on the E-704 data and, therefore, have extensive responsibilities on the E-683 run and for analysis on E-704. 

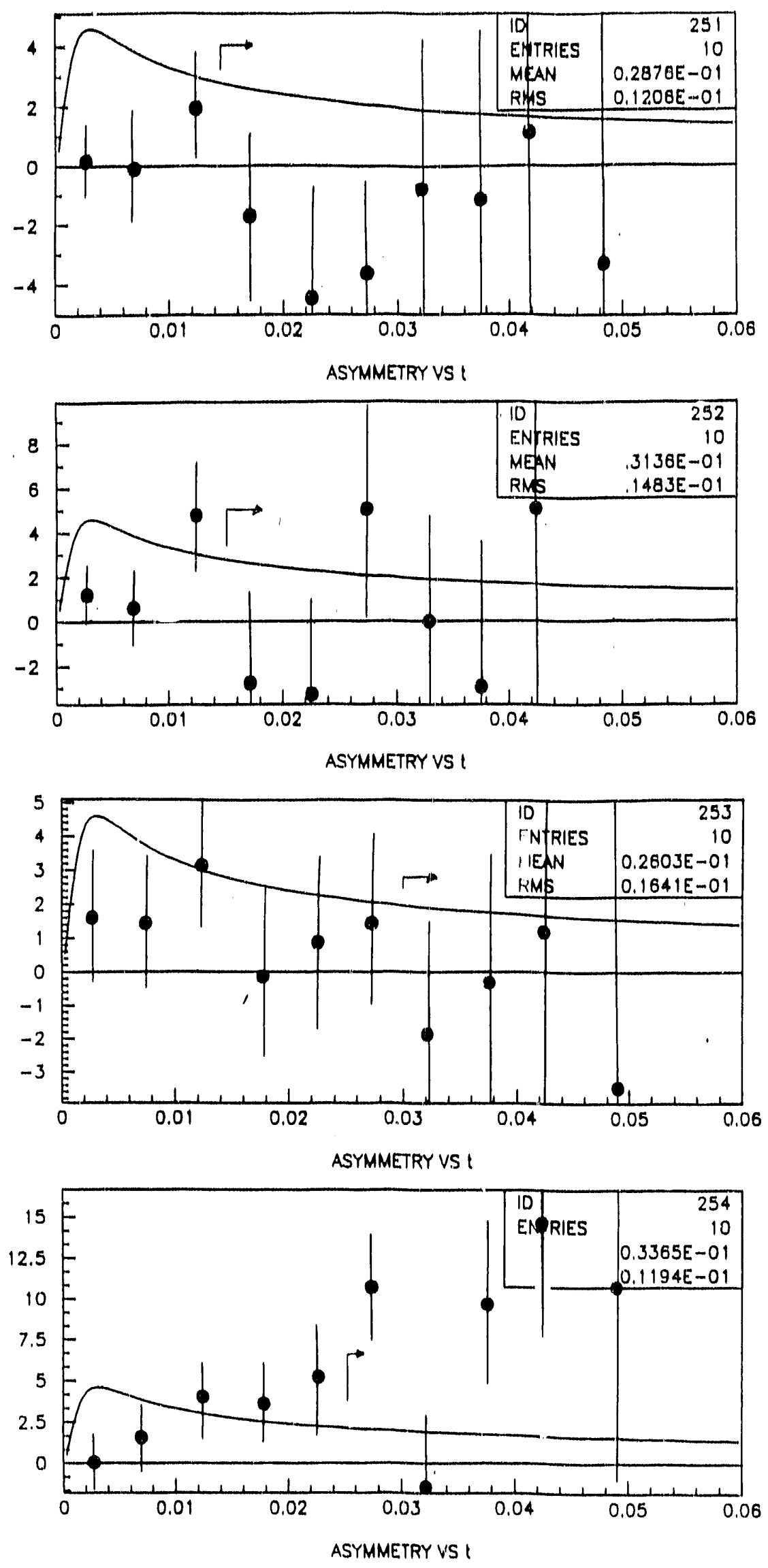

Figures 1a-d. 

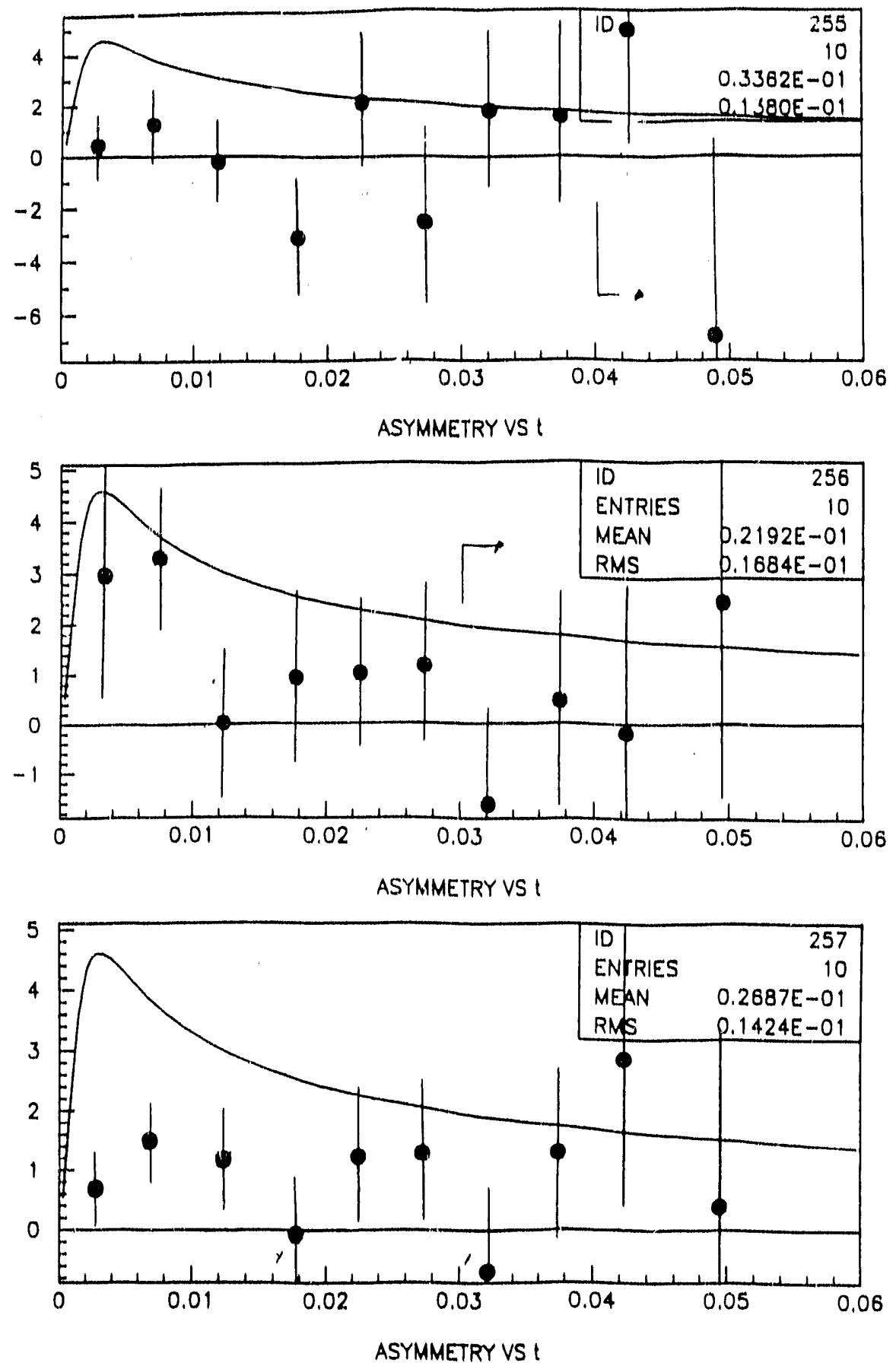

Figures le-g. 
Z-distribution
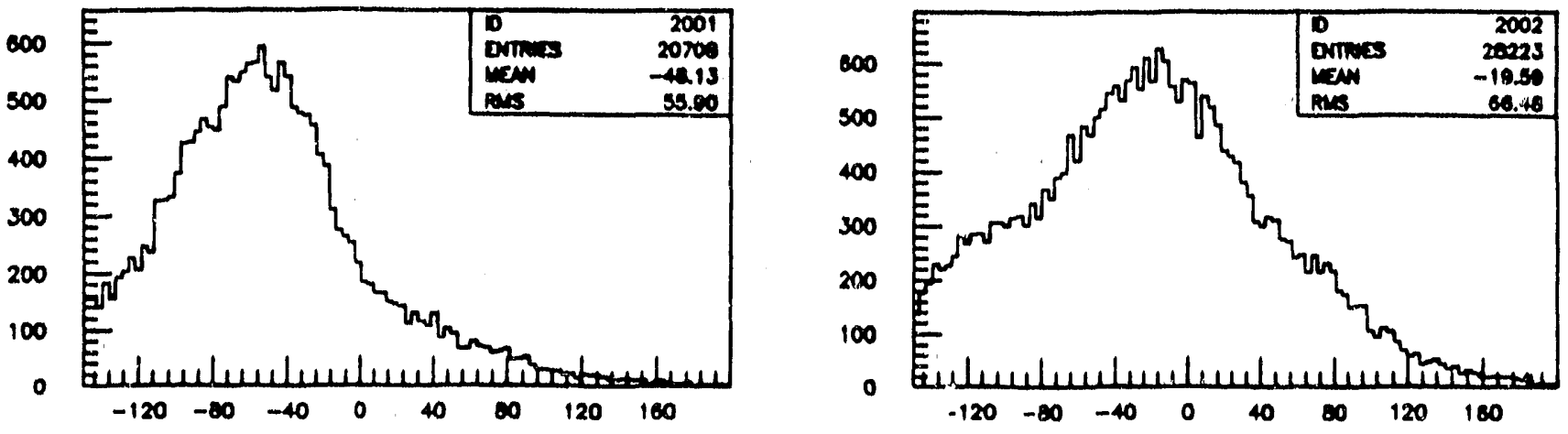

VERTEX PER TARGET 1

VERTEX PER TARGET 2
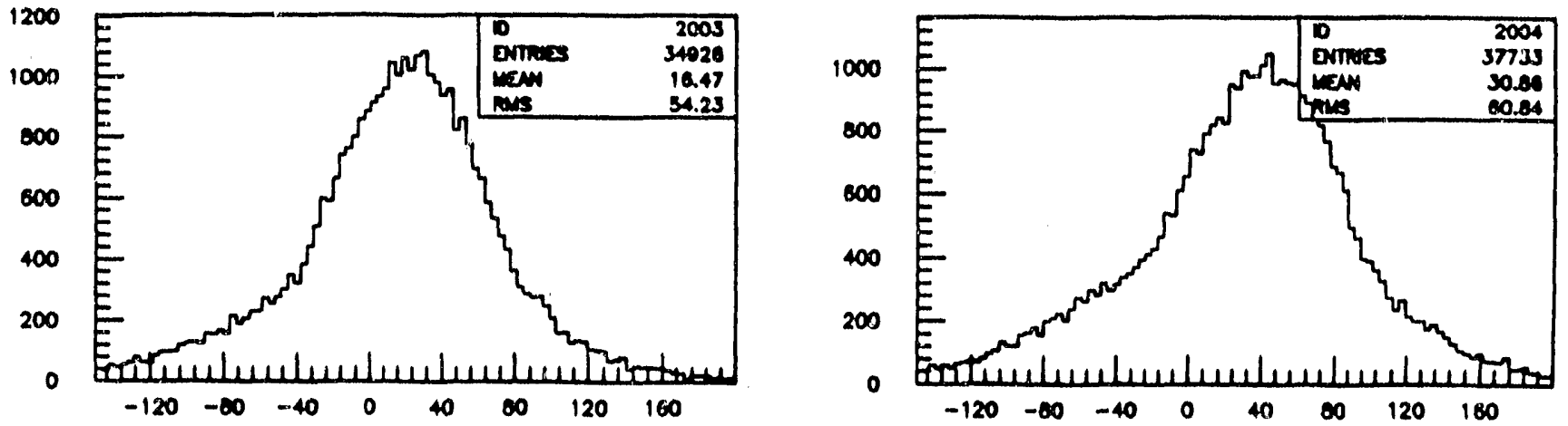

VERTEX PER TARGET 3
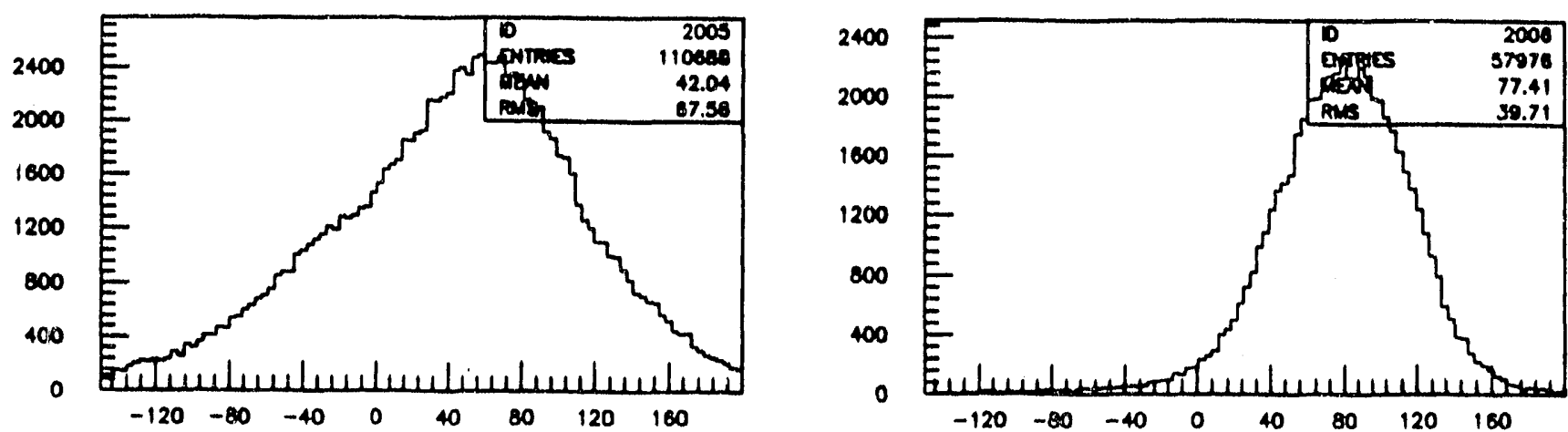

VERTEX PER TARGET 5

Figure 2a. 


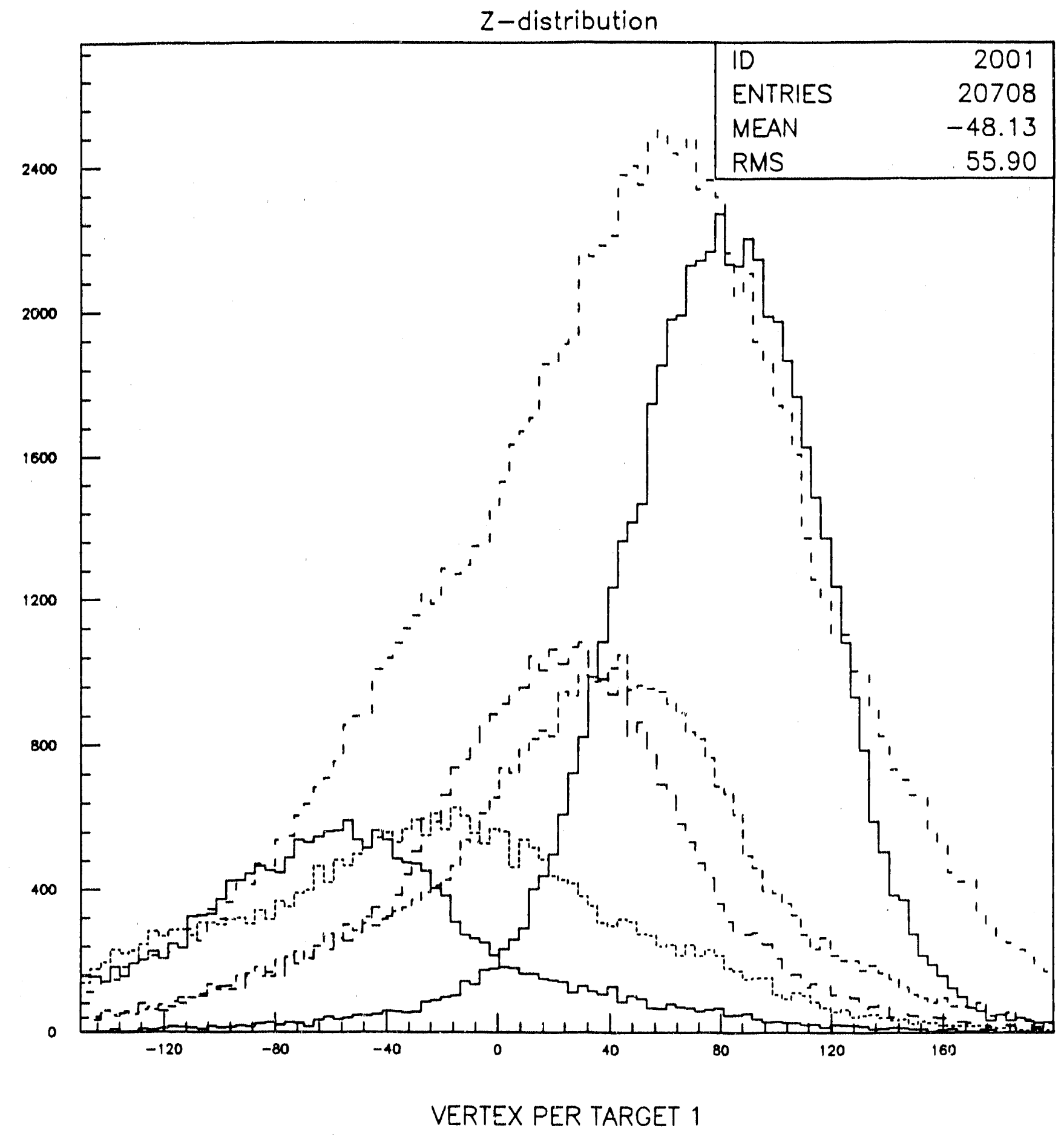

Figure $2 b$. 


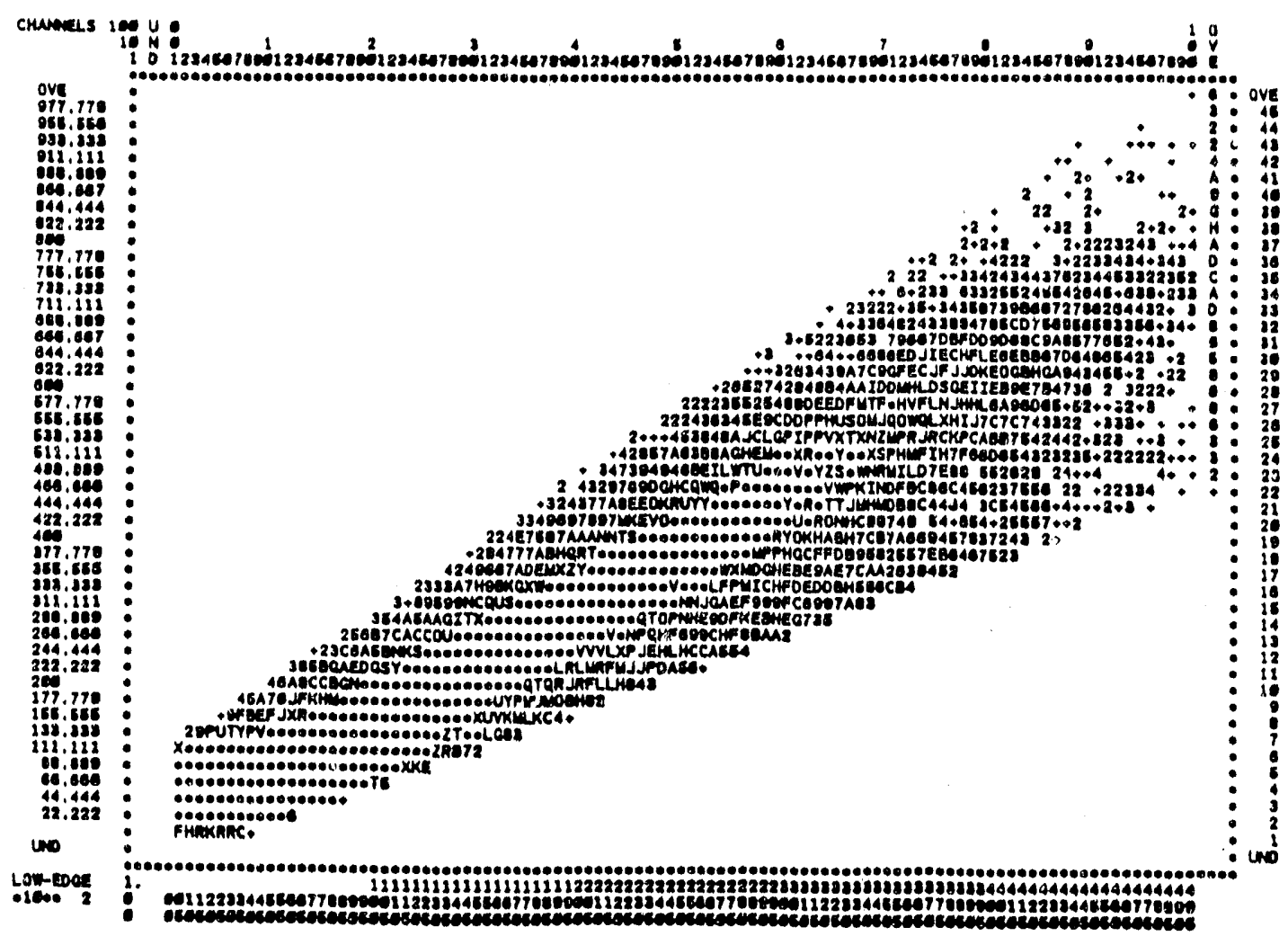

Figure 3a. Target no. 3.

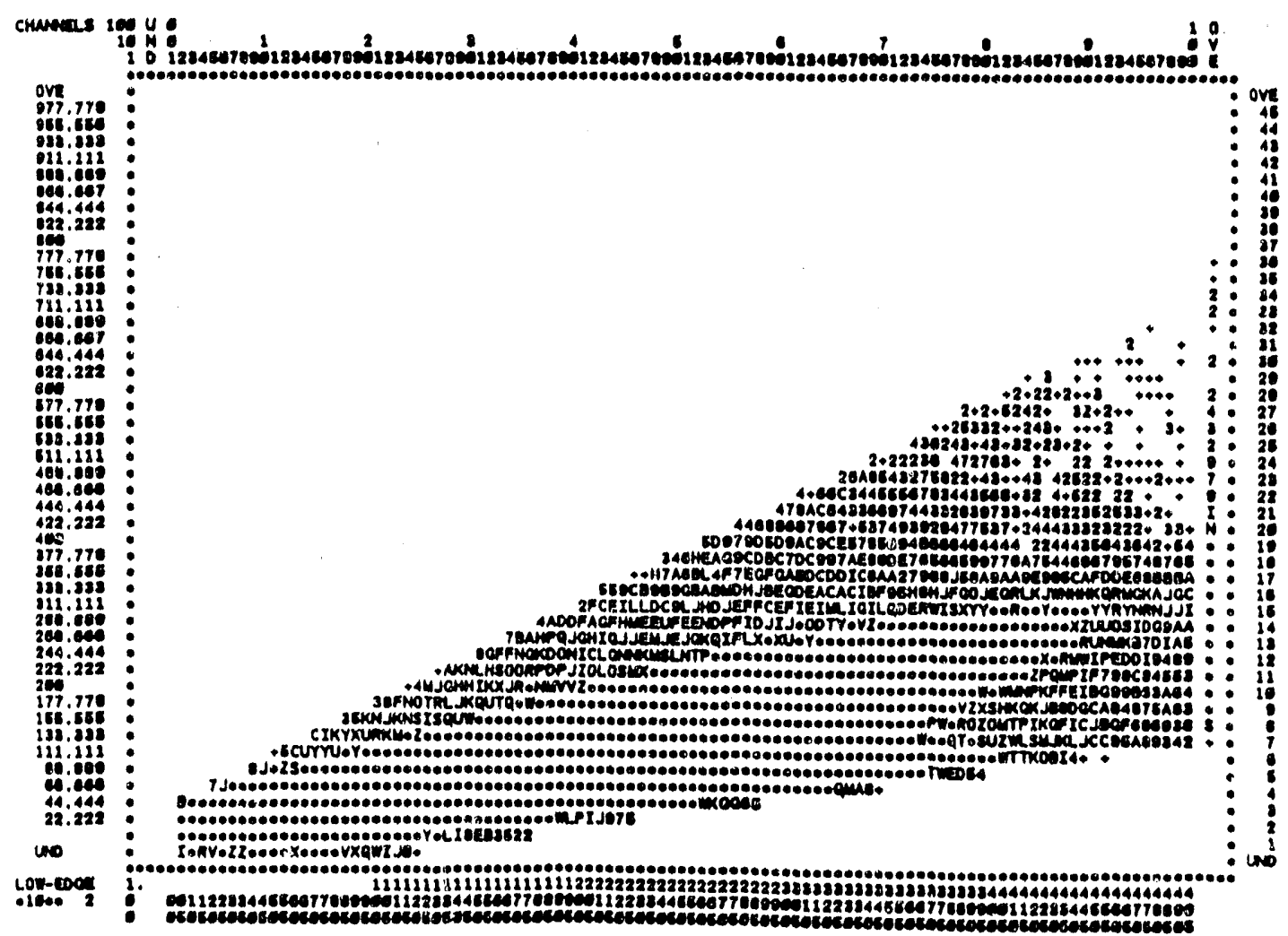

Figure $3 b$. Target no. 6 .

Figures $3 \mathrm{a}-\mathrm{b}$. Four-momentum transfer $|\mathrm{t}|$ correlations with the slow component of the target pulse. 
LEAST SQUARE FITS

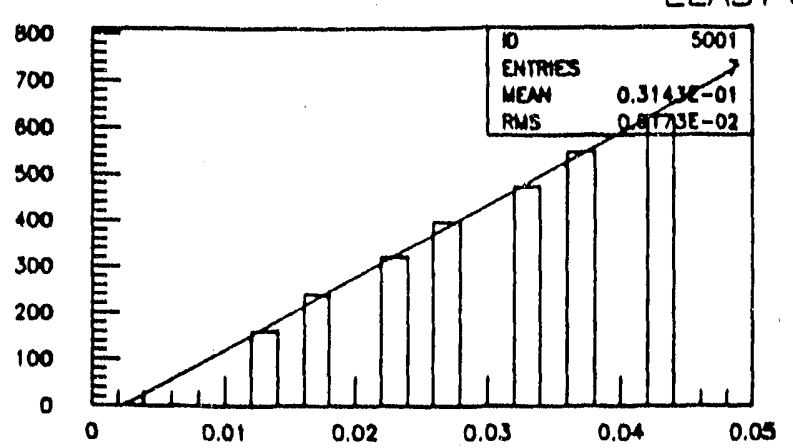

LEAST SQUARES FIT T1

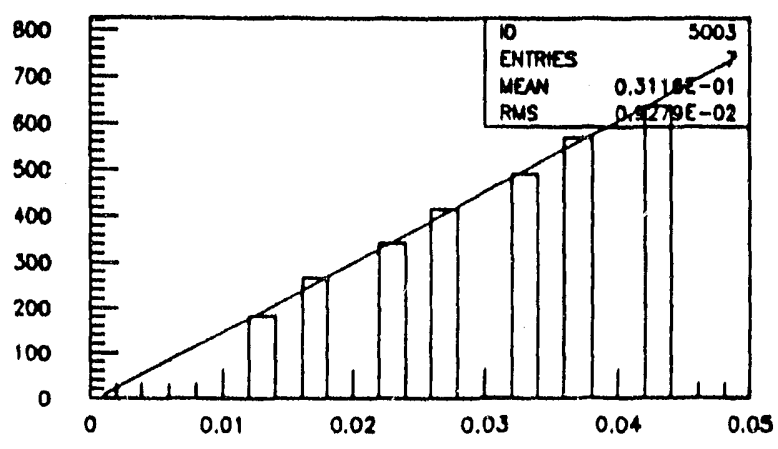

LEAST SQUARES FIT T3

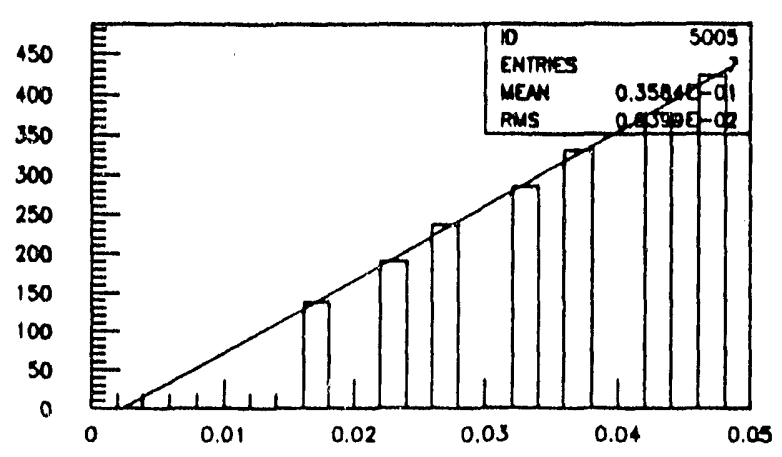

LEAST SQUARES FIT T5

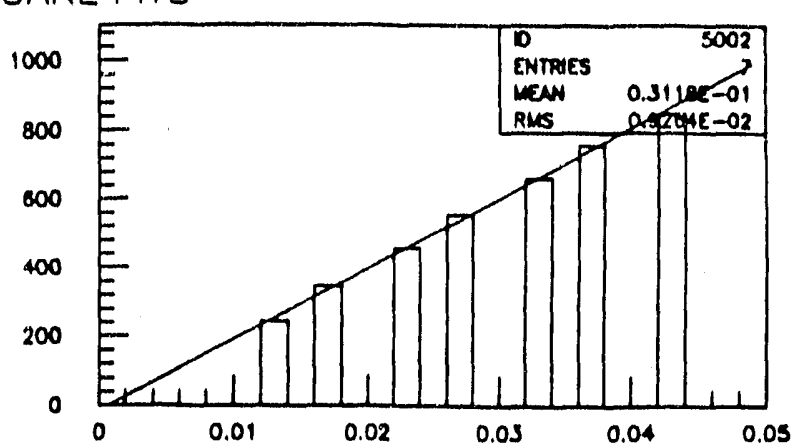

LEAST SQUARES FIT T2

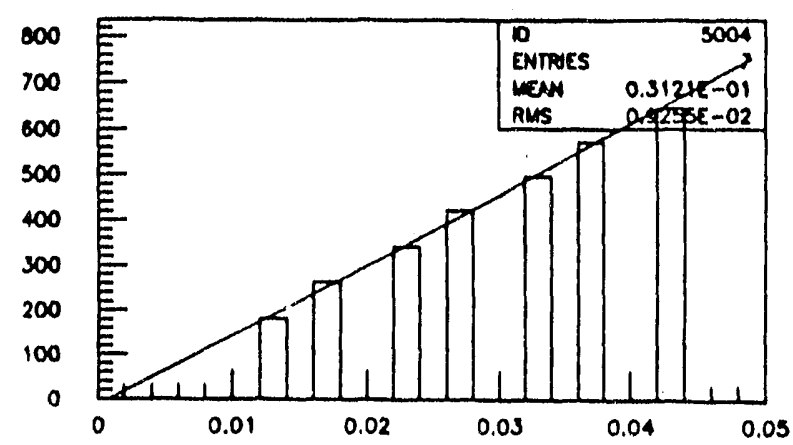

LEAST SQUARES FIT T4

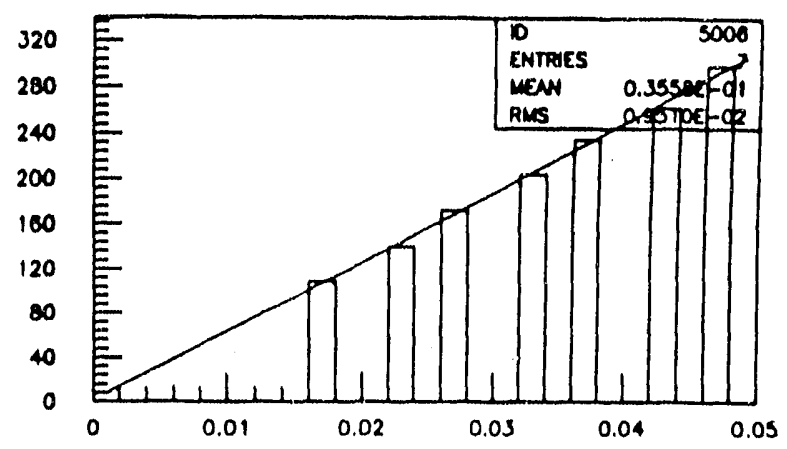

LEAST SQUARES FIT T6

Figure 4a. Least-squares fit for individual targets in $|\mathrm{t}|$ bins of 0.005 $(\mathrm{GeV} / \mathrm{c})^{2}$. 


\section{GAUSSIAN FITS}
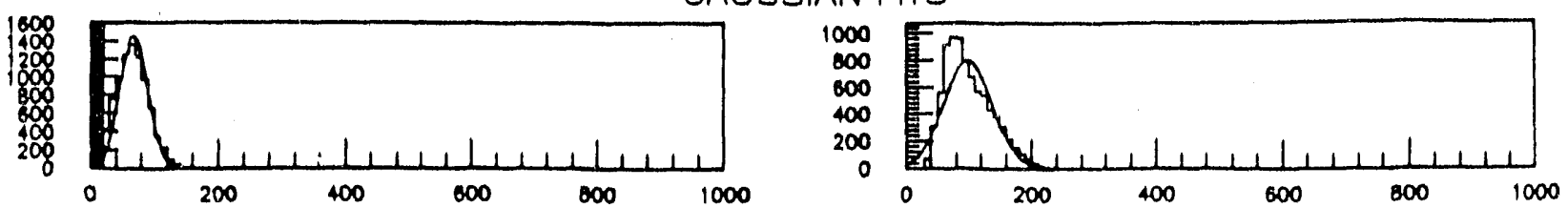

TRGT CUT SLOW 3

TRGT CUT SLOW 3
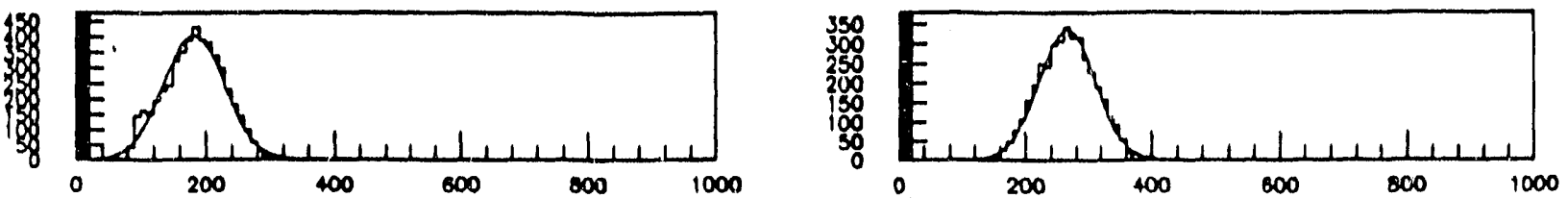

TRGT CUT SLOW 3

TRGT CUT SLOW 3
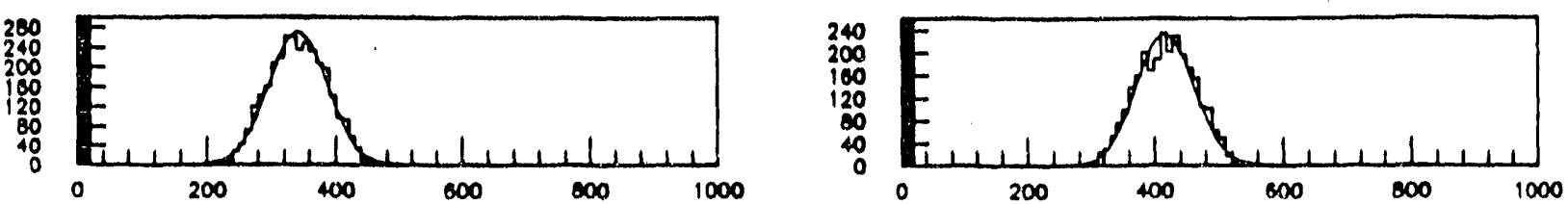

TRGT CUT SLOW 3

TRGT CUT SLOW 3
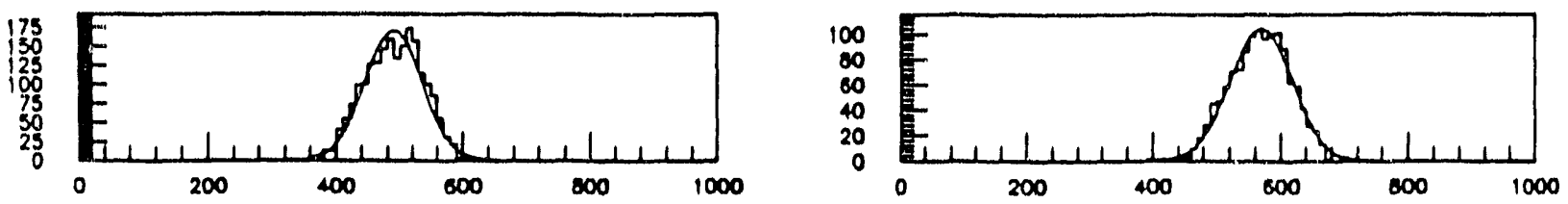

TRGT CUT SLOW 3

TRGT CUT SLOW 3

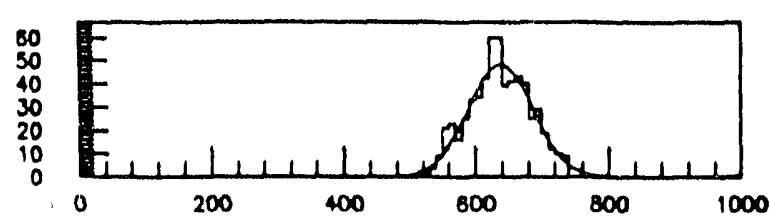

TRGT CUT SLOW 3

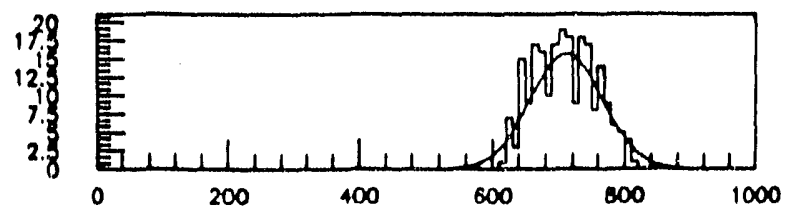

TRGT CUT SLOW 3

Figure 4b. Gaussian fits in slow component ADC counts for each $|\mathrm{t}|$ bin $\left(0.005(\mathrm{GeV} / \mathrm{c})^{2}\right.$ wide) for target no. 3 . 


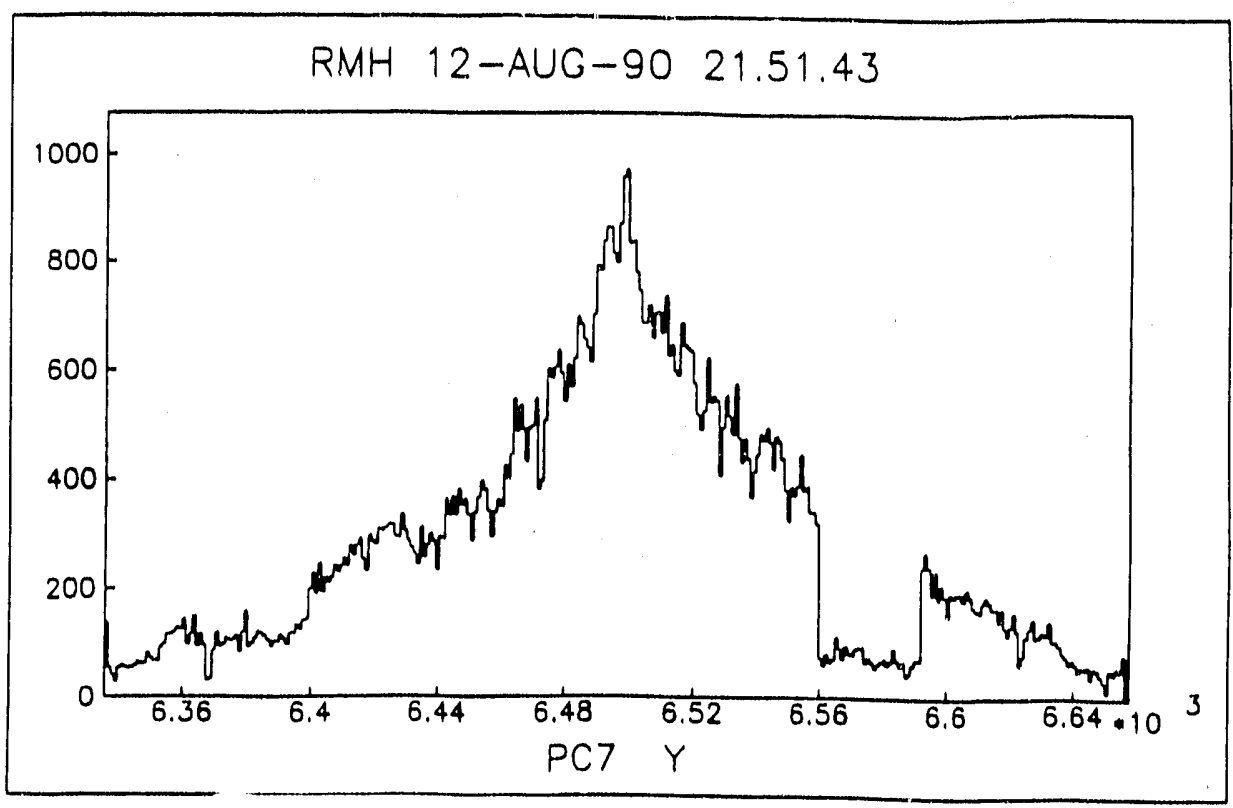

Figure 5a.

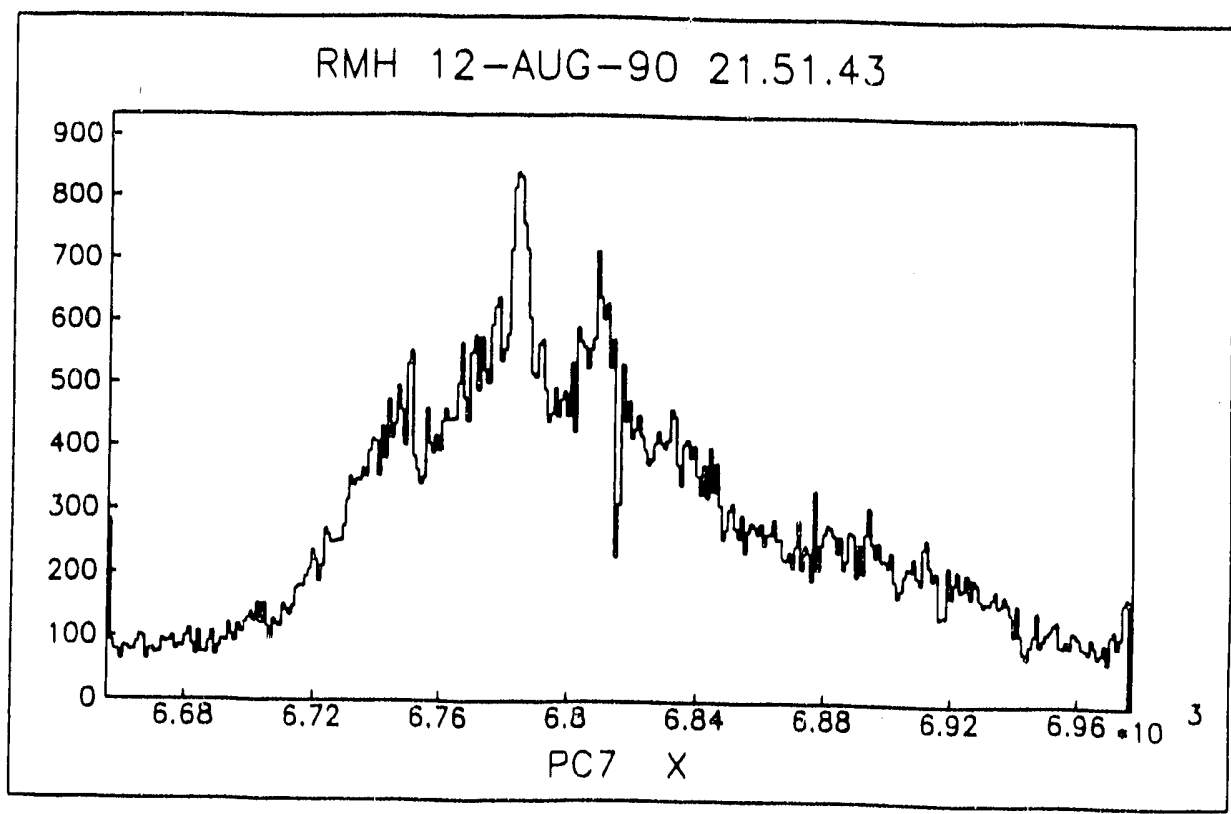

Figure 5b.

Figures 5a-d. Histograms of Iowa MWPC/Forward Spectrometer in E704. 


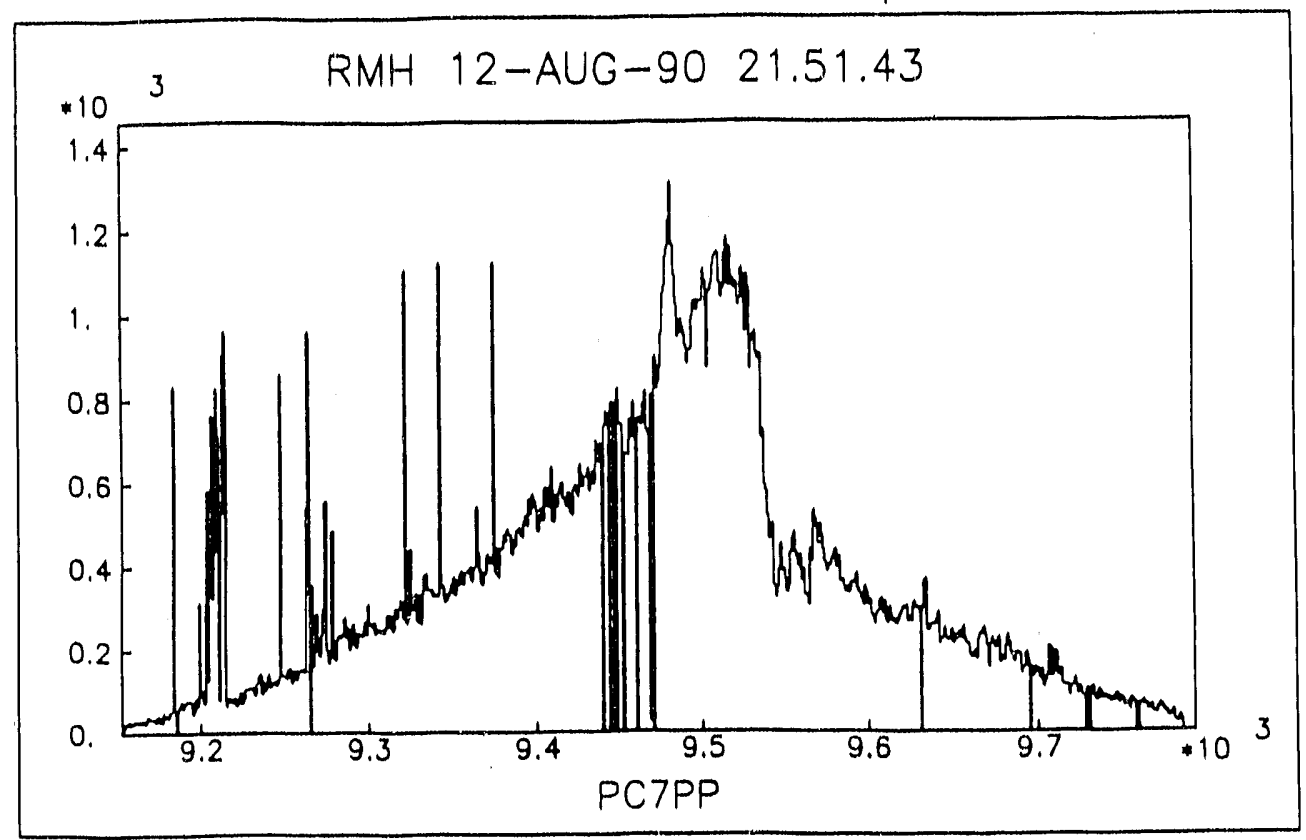

Figure 5c.

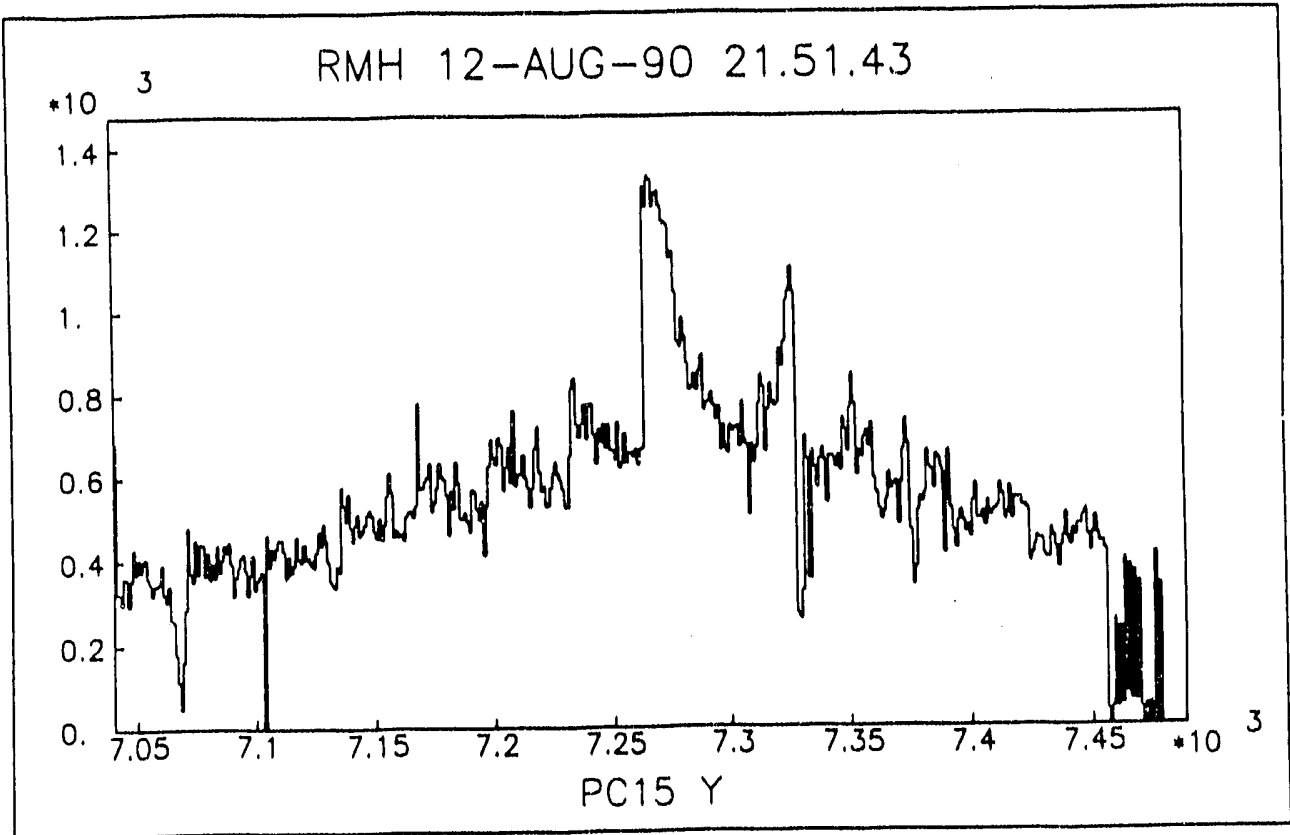

Figure 5d. 
LAMBDA DECAY SPACE
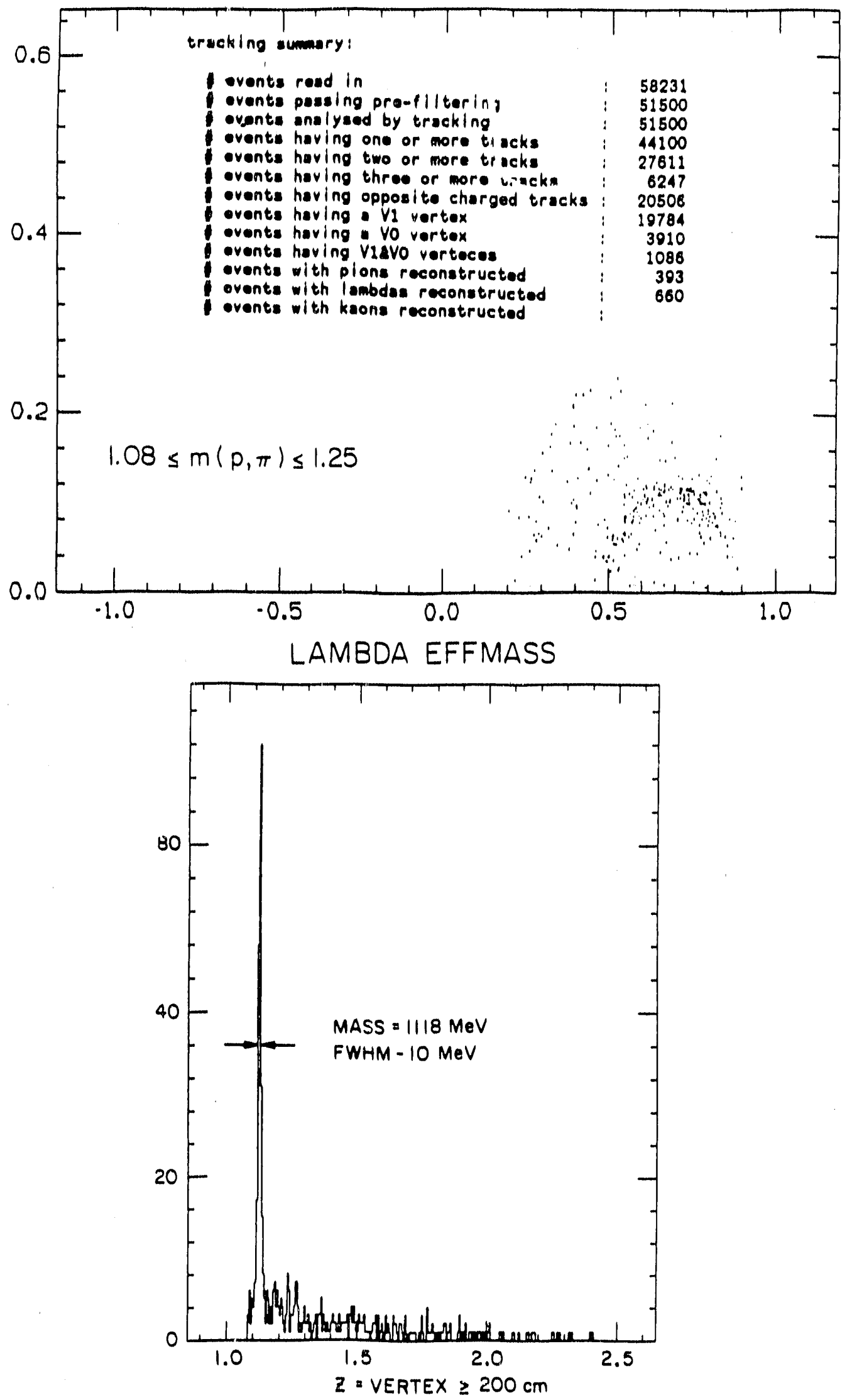

Figures 6a-6b. 

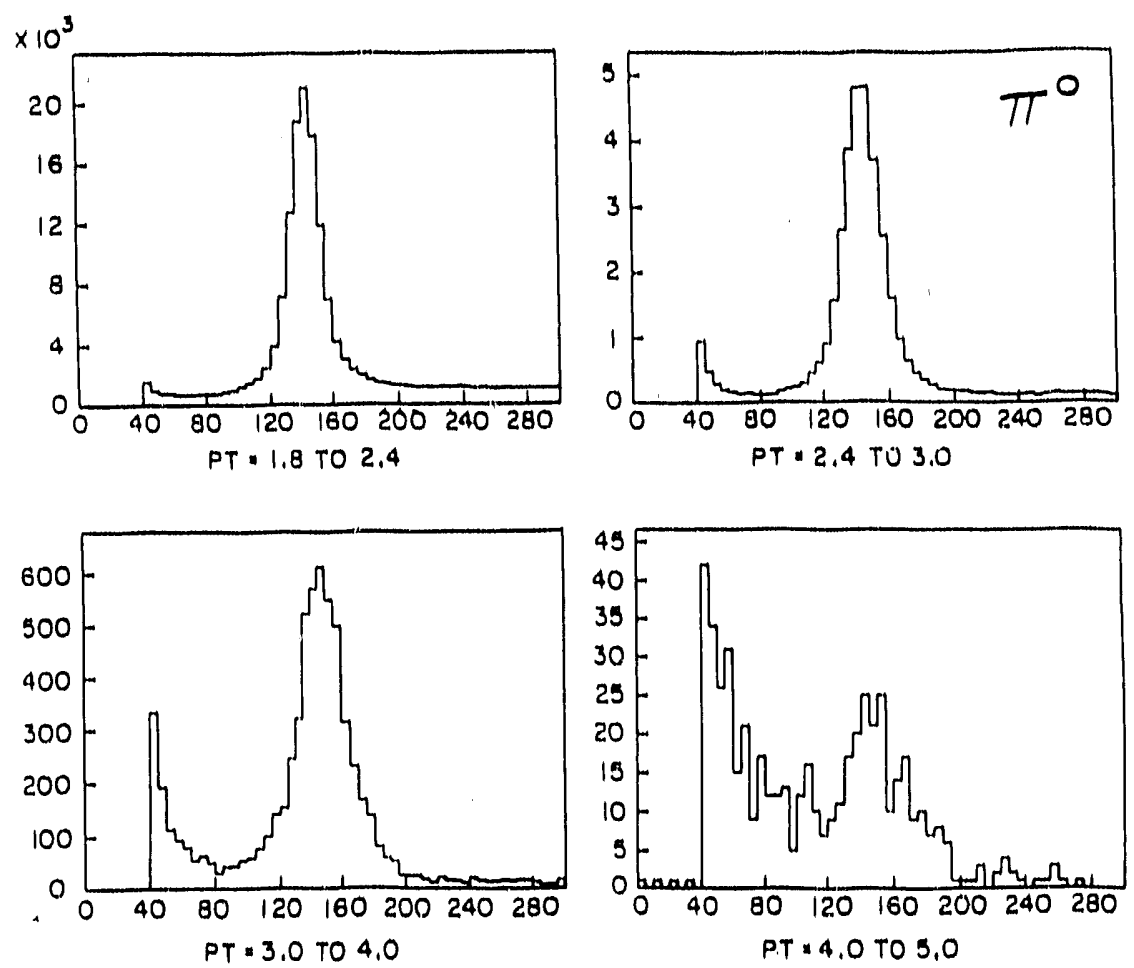

Figure 7. $\pi^{0}$ mass distributions for different $p_{t}$-regions at $x_{f}=0$.

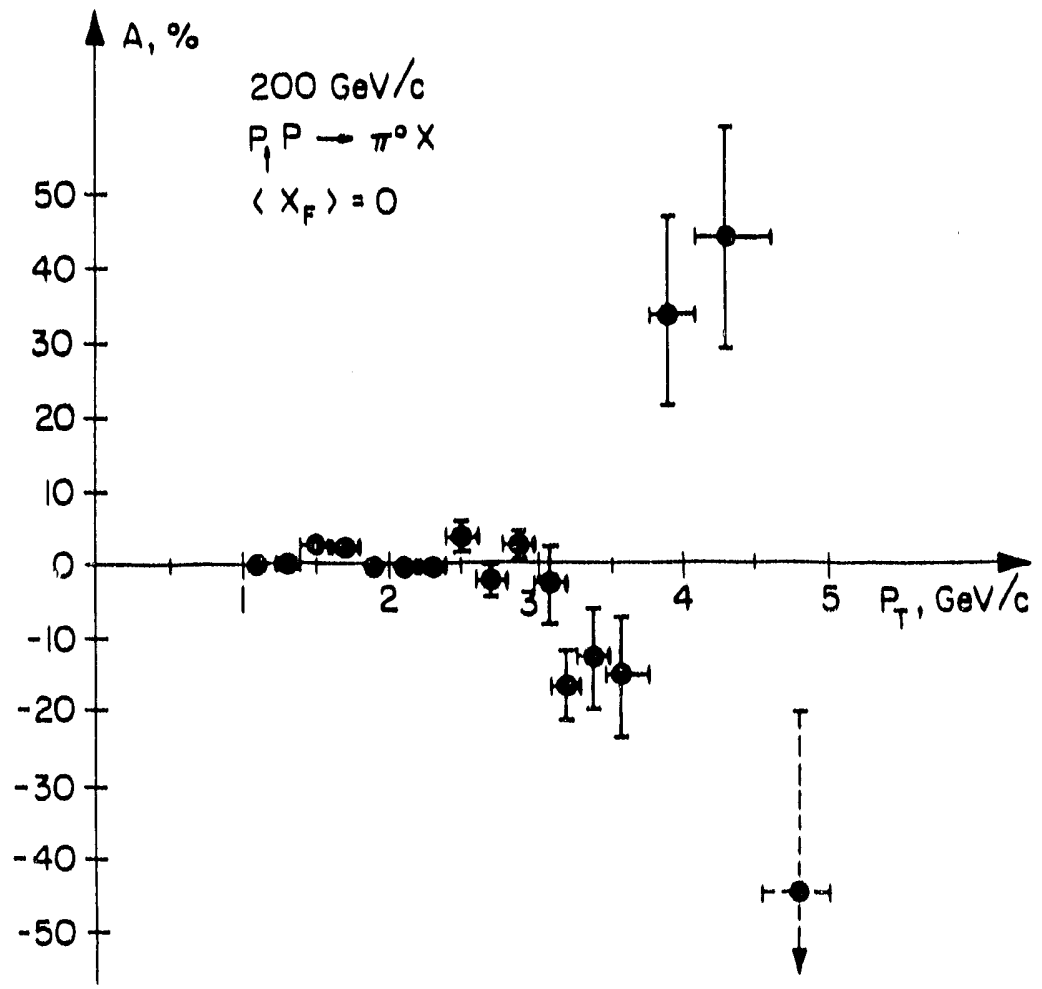

Figure 8. Asymmetry in the reacton $\mathrm{p} \uparrow+\mathrm{p} \rightarrow \pi^{0}+\mathrm{X}$ as a function of $p_{t}$ at $x_{f}=0$. 


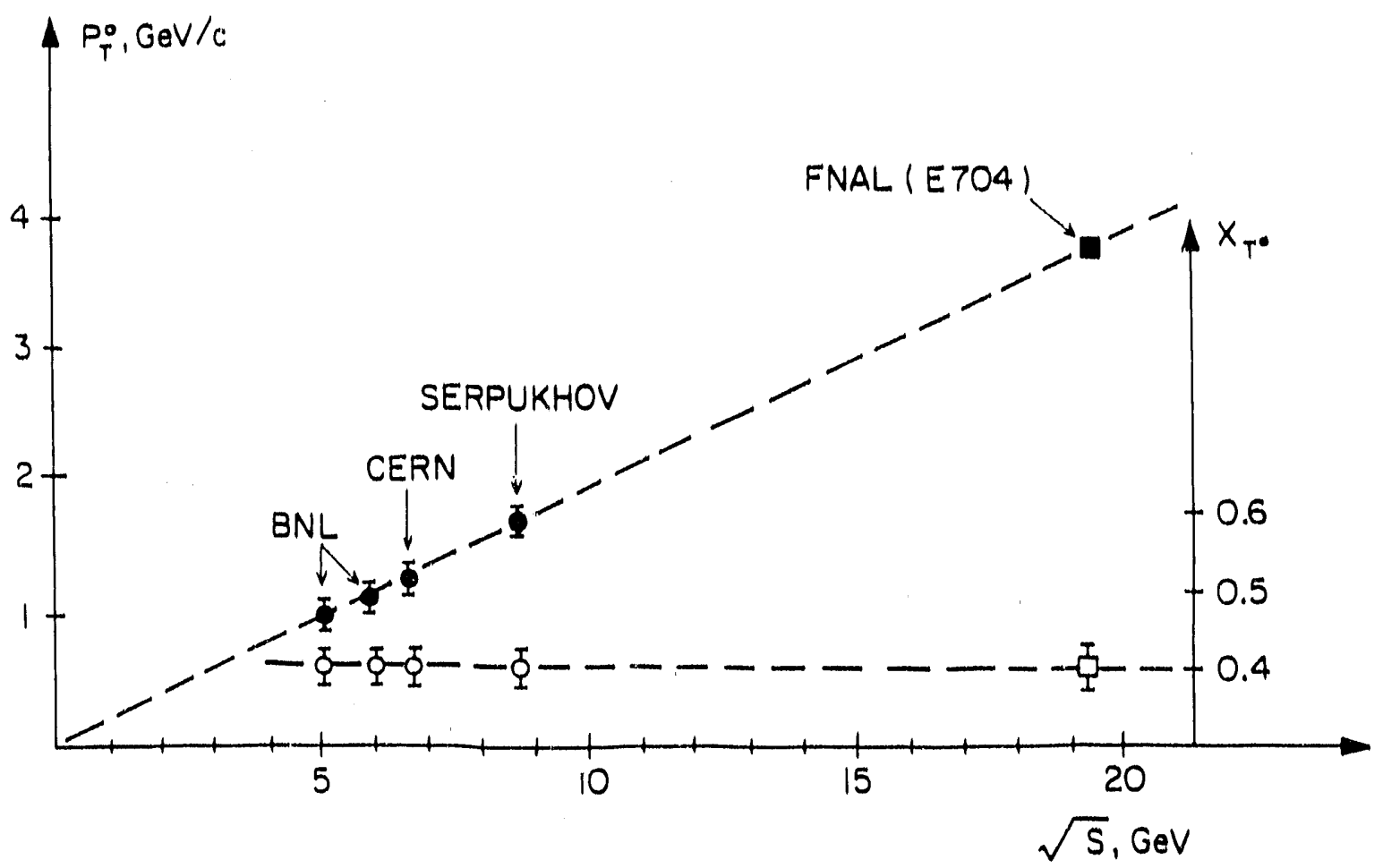

Figure 9. Cross-over points $p_{t}{ }^{0}$ and $x_{t}{ }^{0}$ versus $\checkmark s$ for different experiments. Dashed lines present the linear approximations.
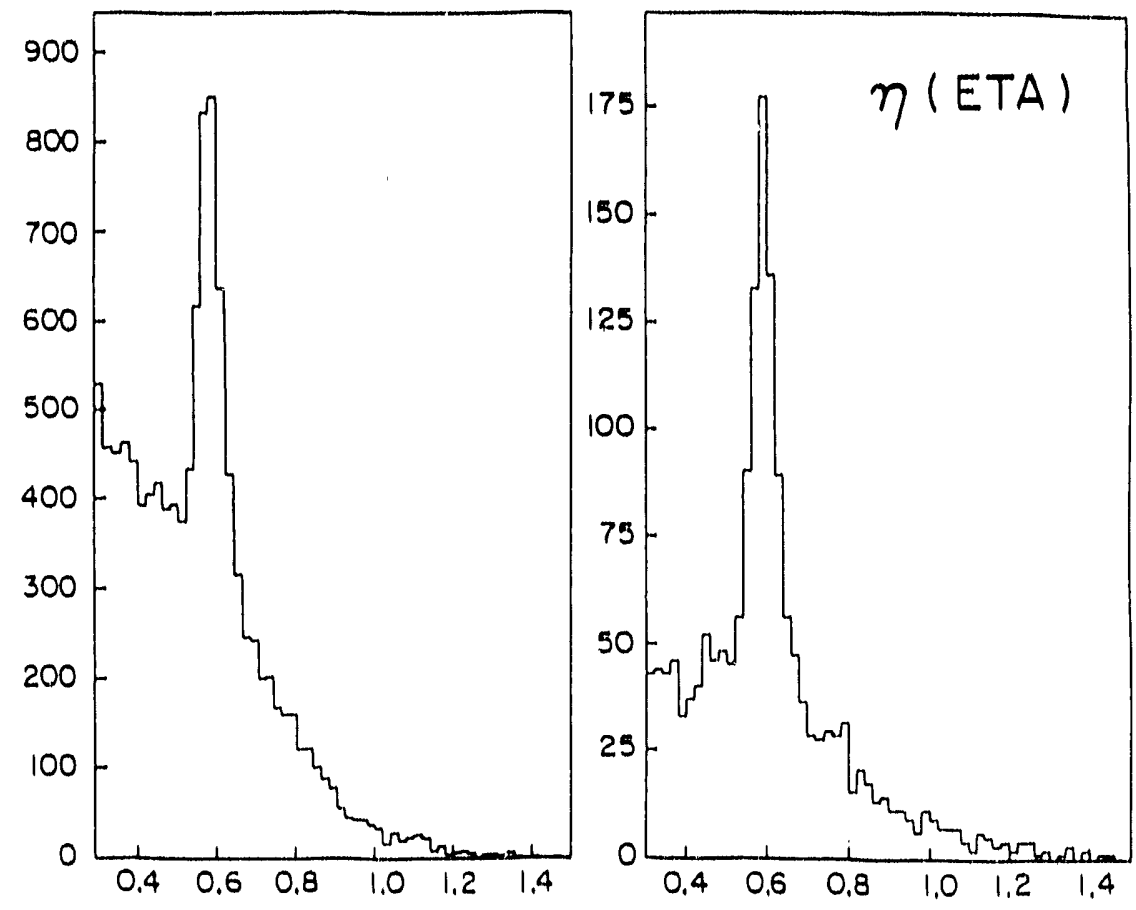

$$
\mathrm{p}_{\mathrm{t}}=2.4 \text { to } 3.0
$$$$
\mathrm{p}_{\mathrm{t}}=3.0 \text { to } 4.0
$$

Figure 10. $\eta$ mass distributions for different $\mathrm{p}_{t}$-regions at $\mathrm{x}_{\mathrm{f}}=0$ 


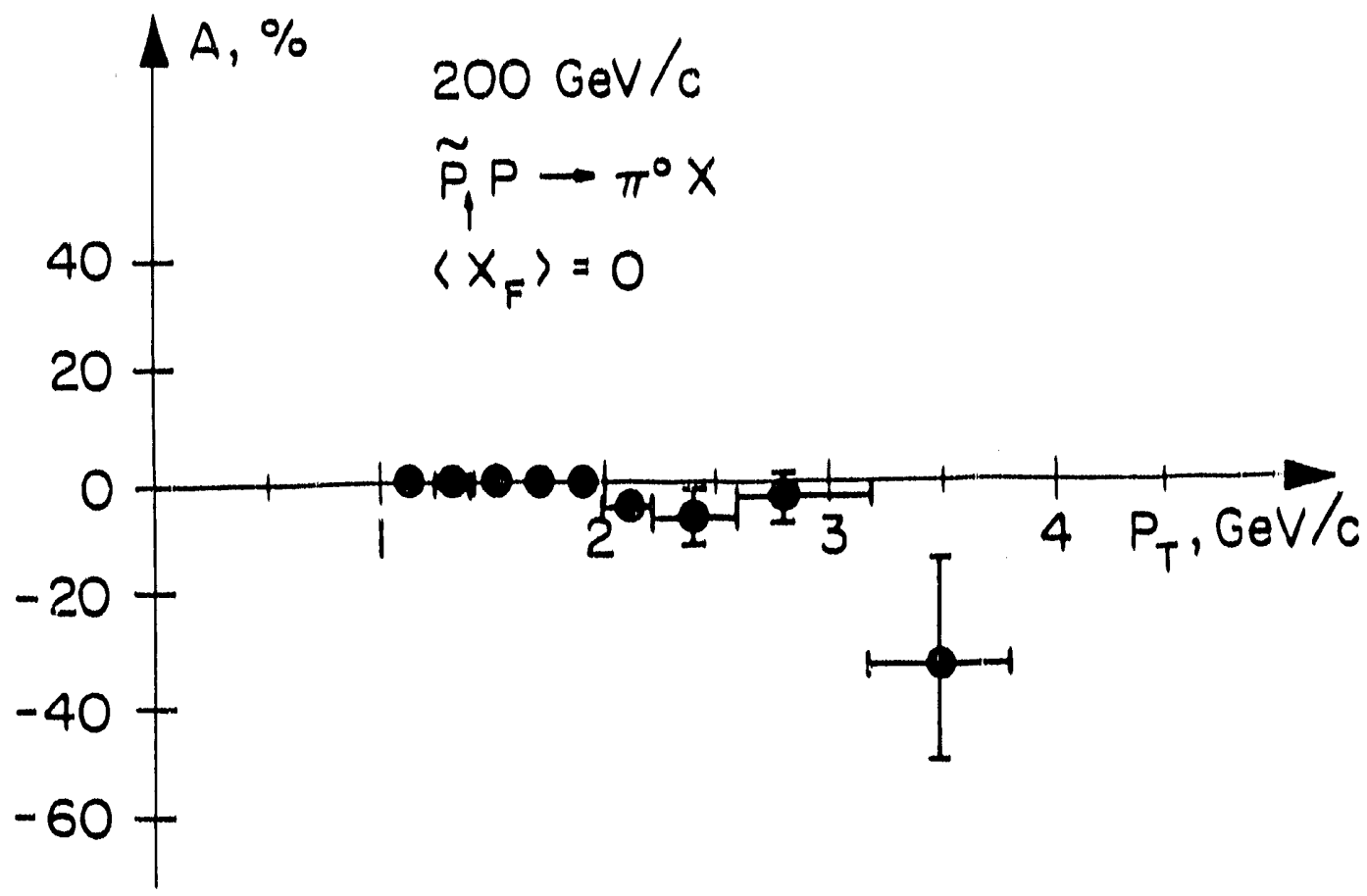

Figure 11a. Asymmetry in the reaction $\overline{\mathrm{p}} \uparrow+\mathrm{p} \rightarrow \pi^{0}+\mathrm{X}$ as a function of $p_{t}$ at $x_{f}=0$.

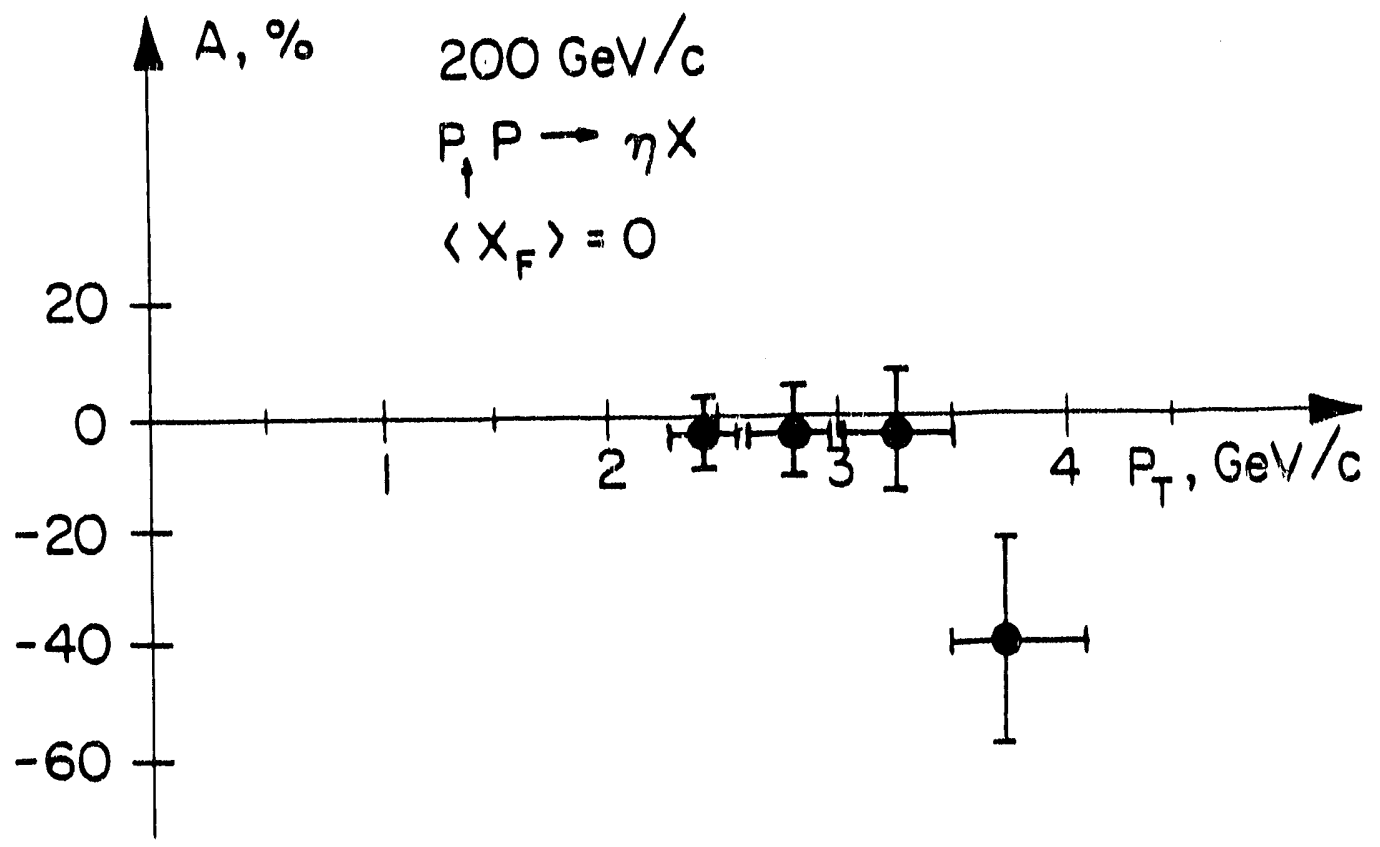

Figure 11b. Asymmetry in the reaction $p \uparrow+p \rightarrow \eta+X$ as a function of $\mathrm{p}_{\mathrm{t}}$ at $\mathrm{x}_{\mathrm{f}}=0$. 

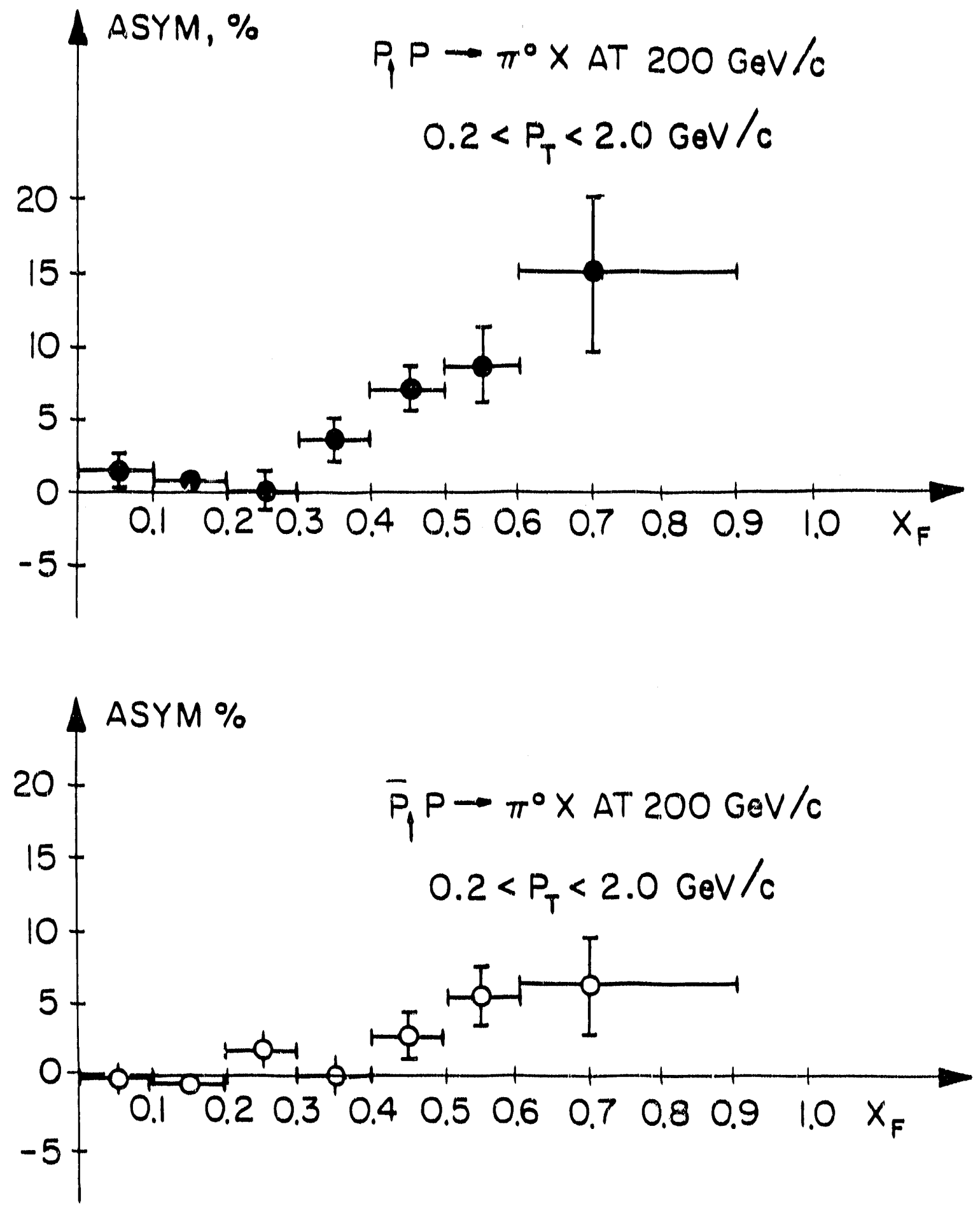

Figure 12. Asymmetries in the reactions $\mathrm{p} \uparrow, \overrightarrow{\mathrm{p}} \uparrow+\mathrm{p} \rightarrow \pi^{0}+\mathrm{X}$ integrated over the $\mathrm{p}_{\mathrm{t}}$-region $0.2<\mathrm{p}_{\mathrm{t}}<2.0 \mathrm{GeV} / \mathrm{c}$. 

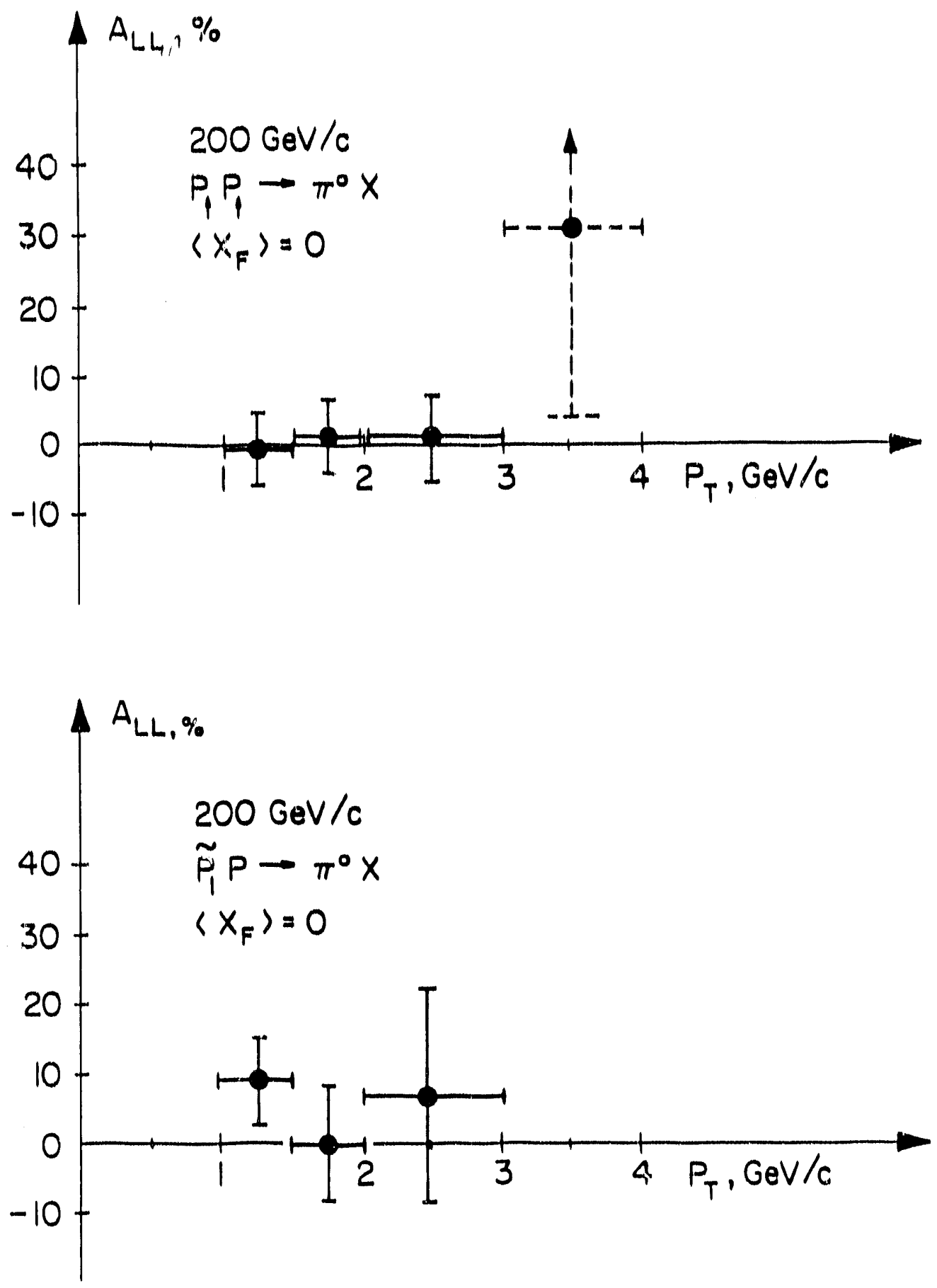

Figure 13. Double spin asymmetries $A_{L L}$ for the reactions $\mathrm{p} \uparrow, \overline{\mathrm{p}} \uparrow+\mathrm{p} \uparrow \rightarrow \pi^{0}+\mathrm{X}$ 


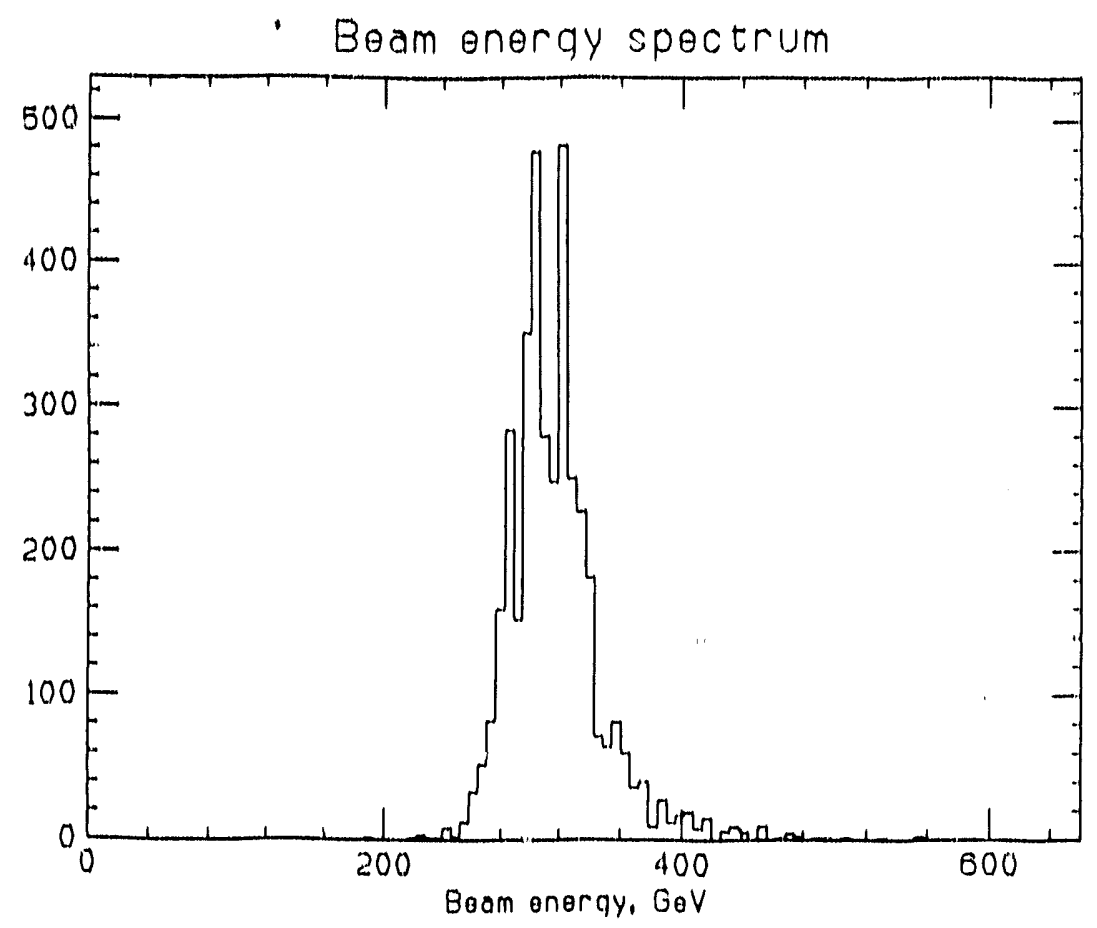

Figure 14.

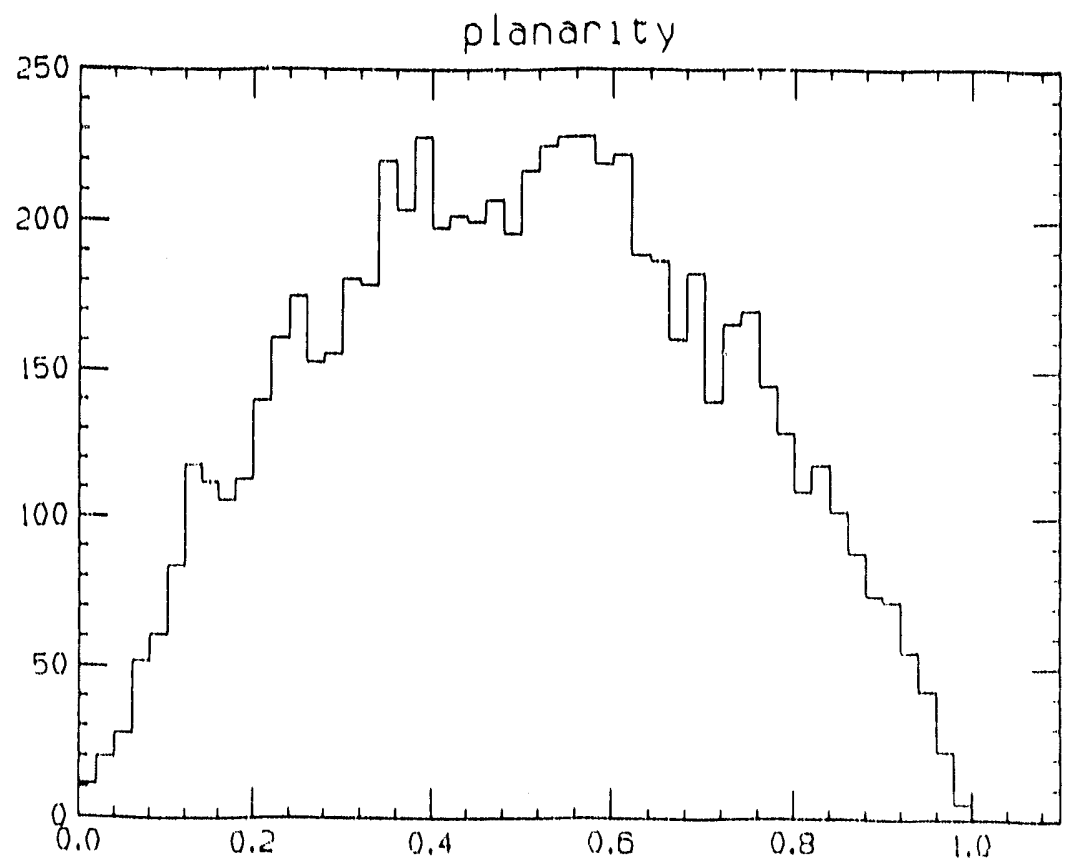

Figure 15 


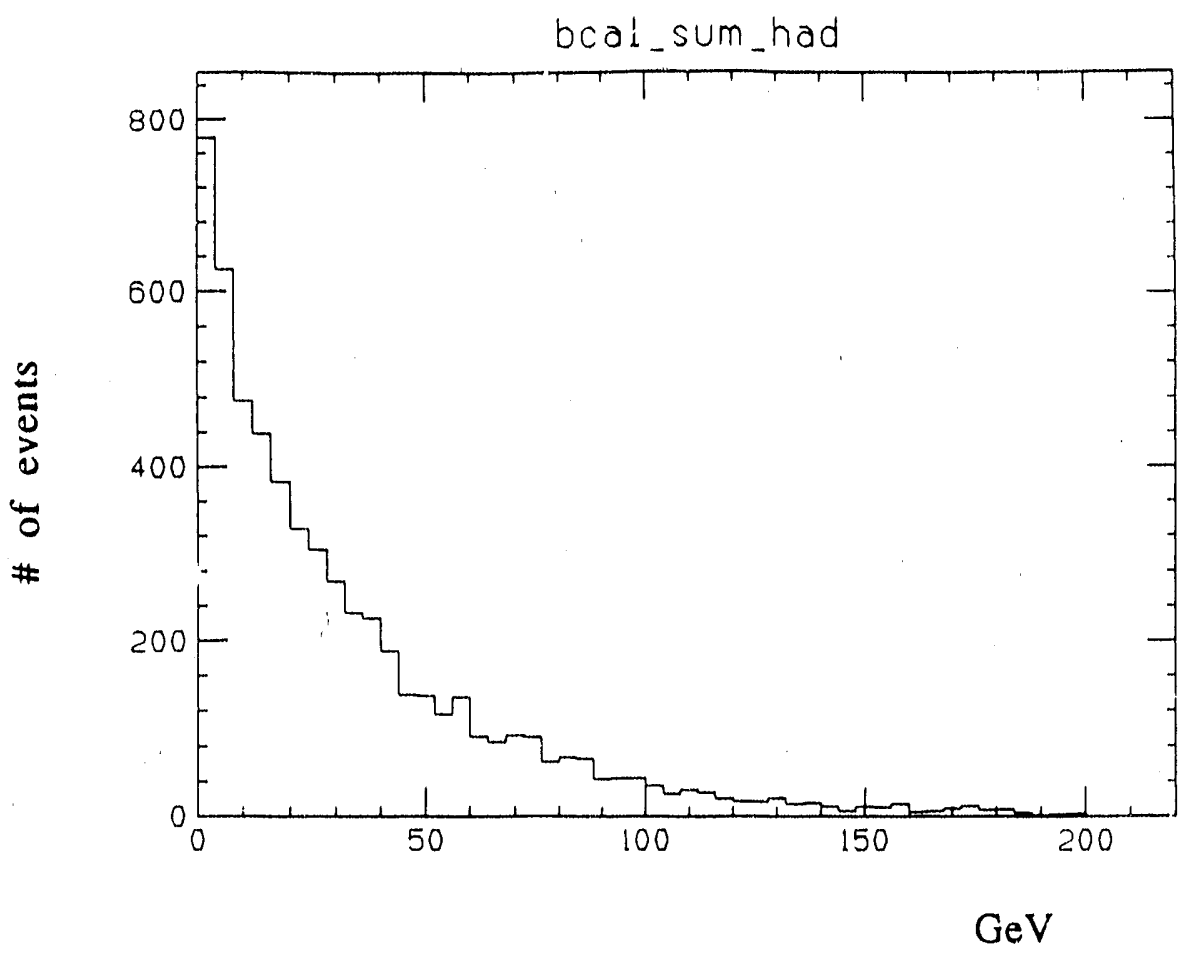

Figure 16a.

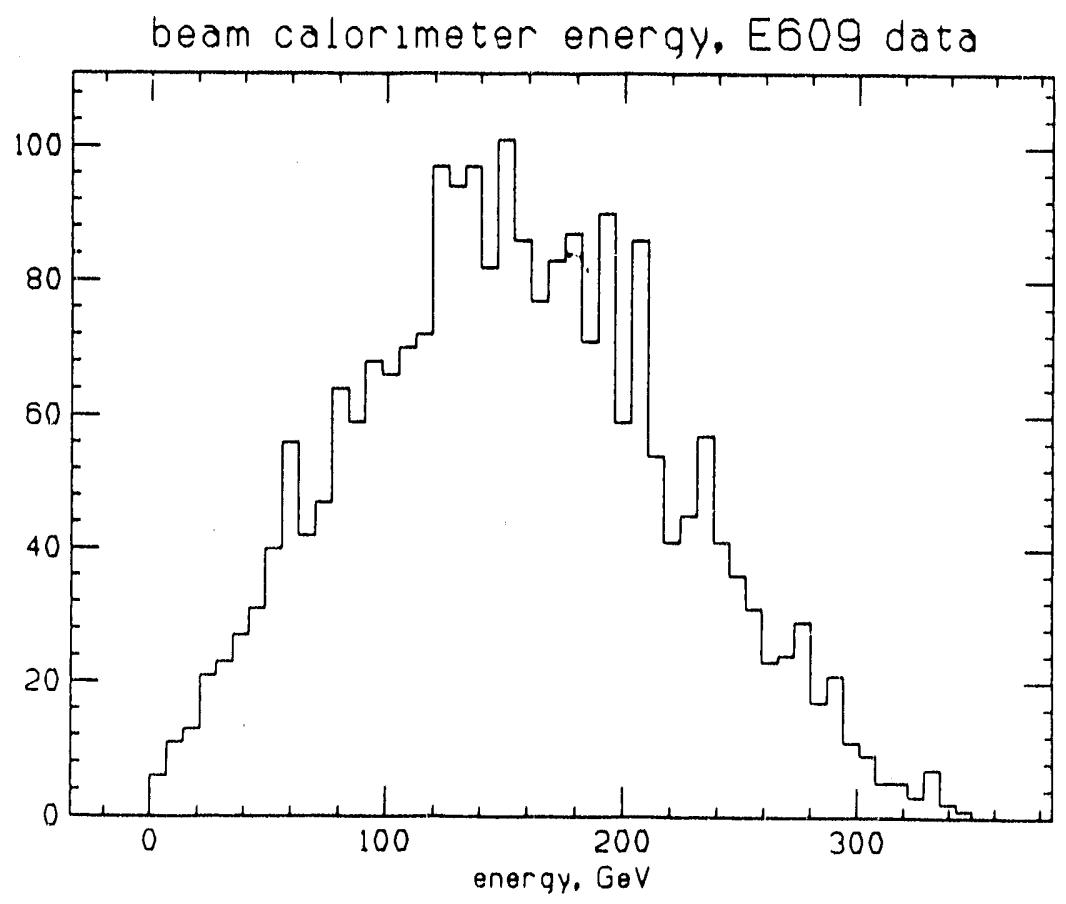

Figure 16b. 

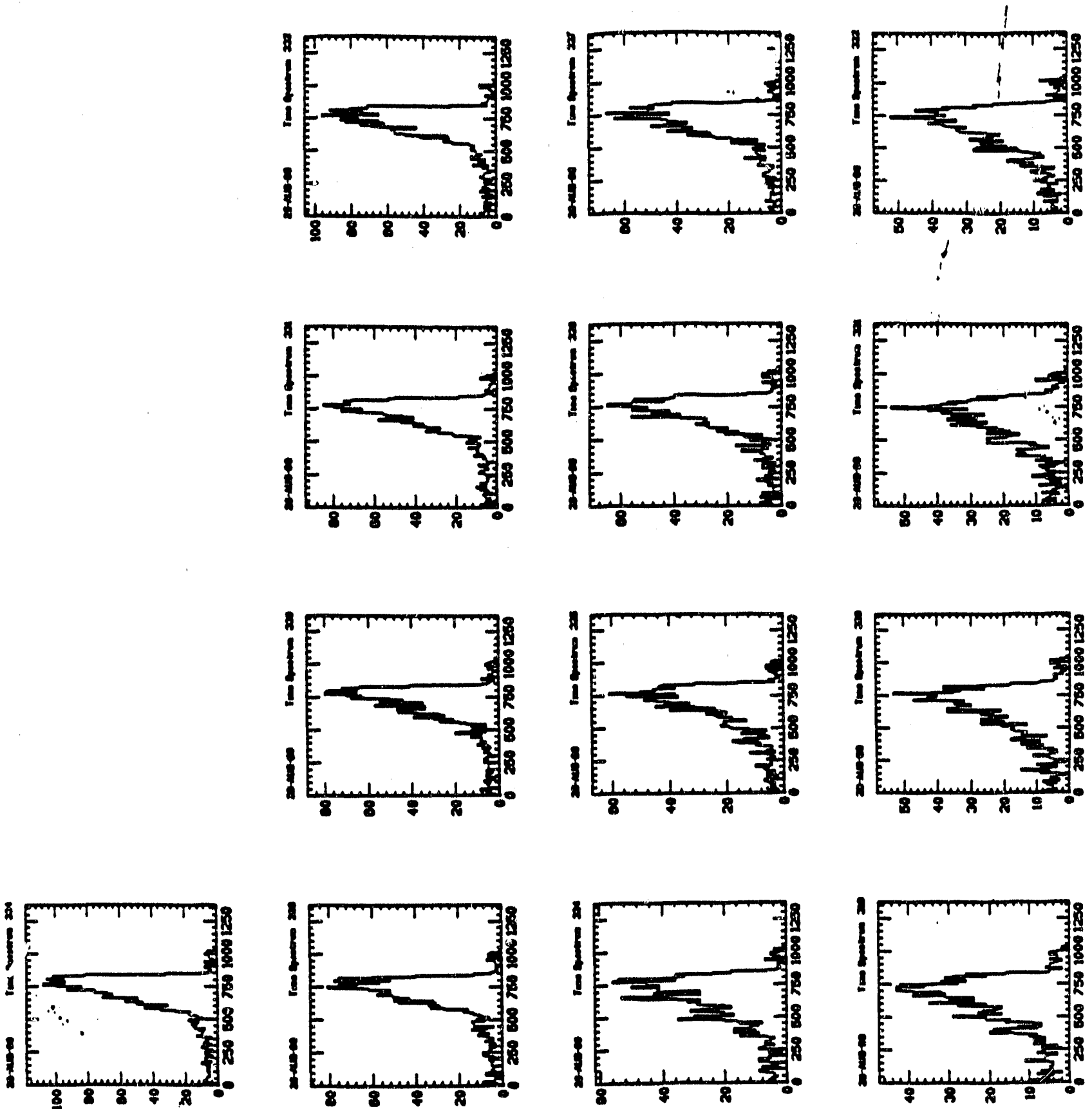

0
$\frac{0}{2}$
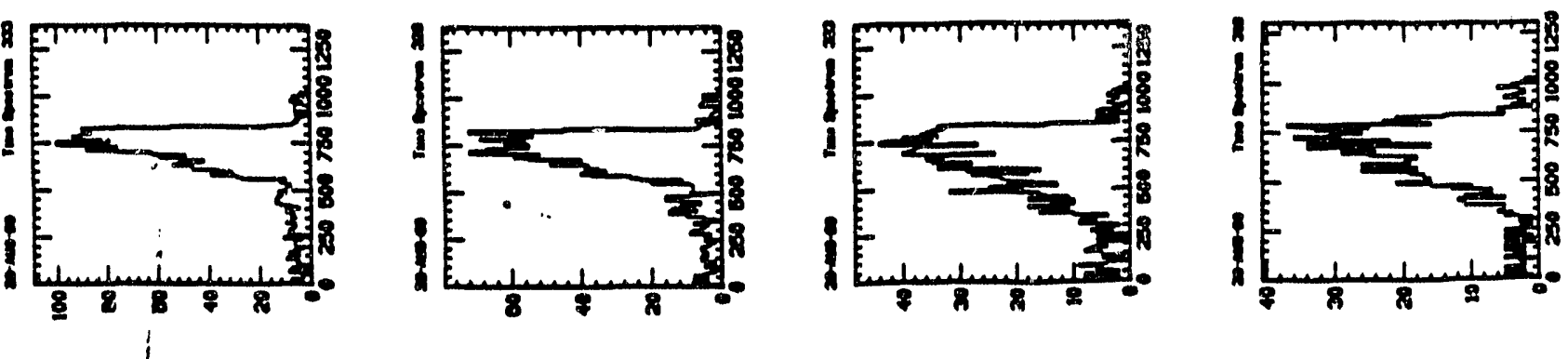

造

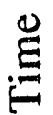

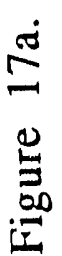




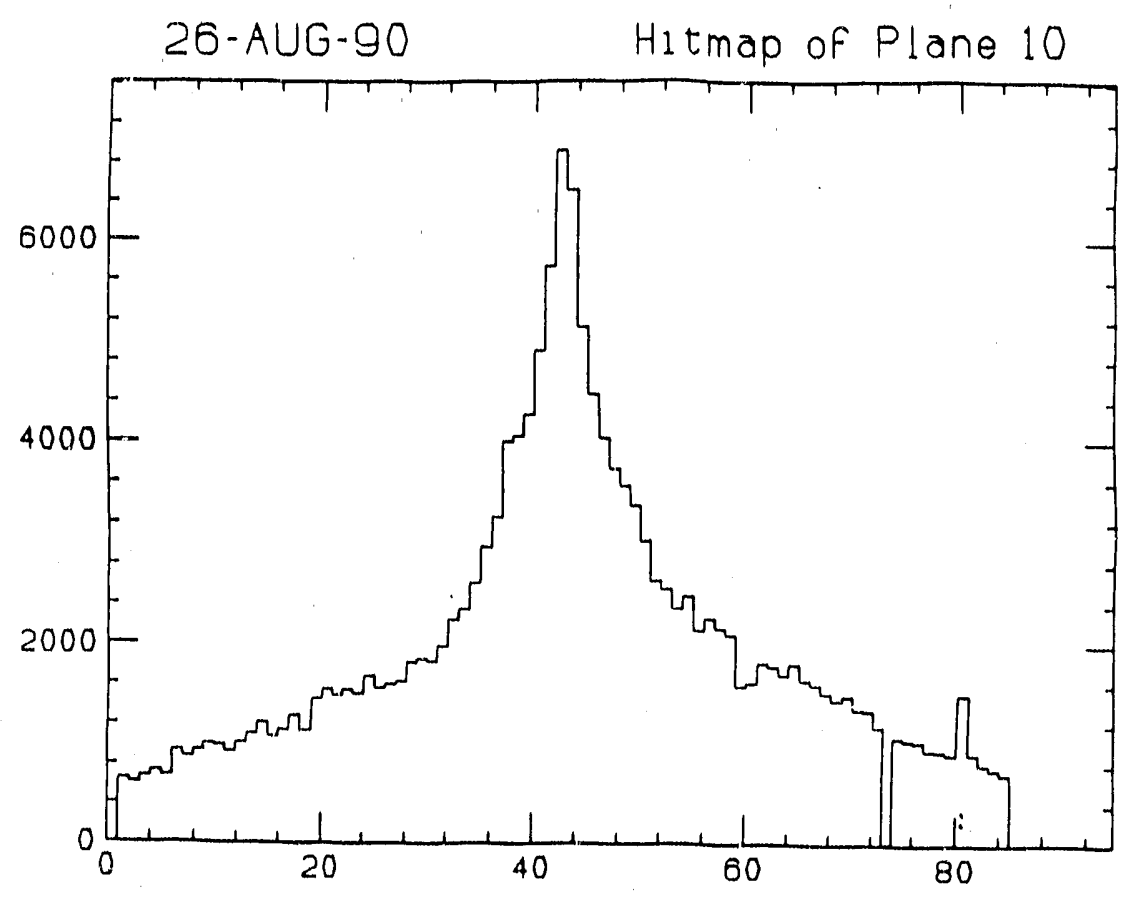

Figure 17b. Hitmap for plane 10 .
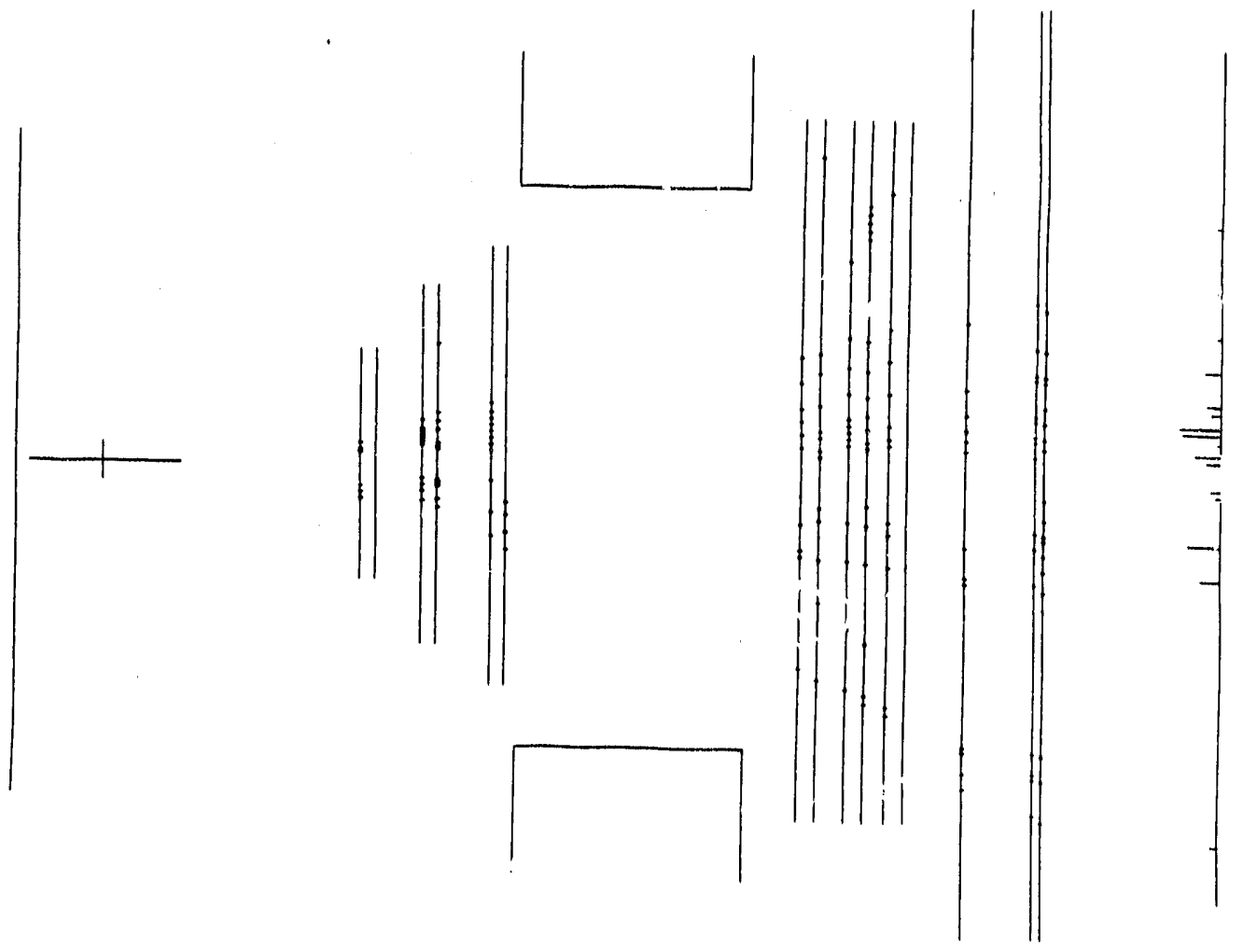

Figure 17c. Online tracking for typical event. 


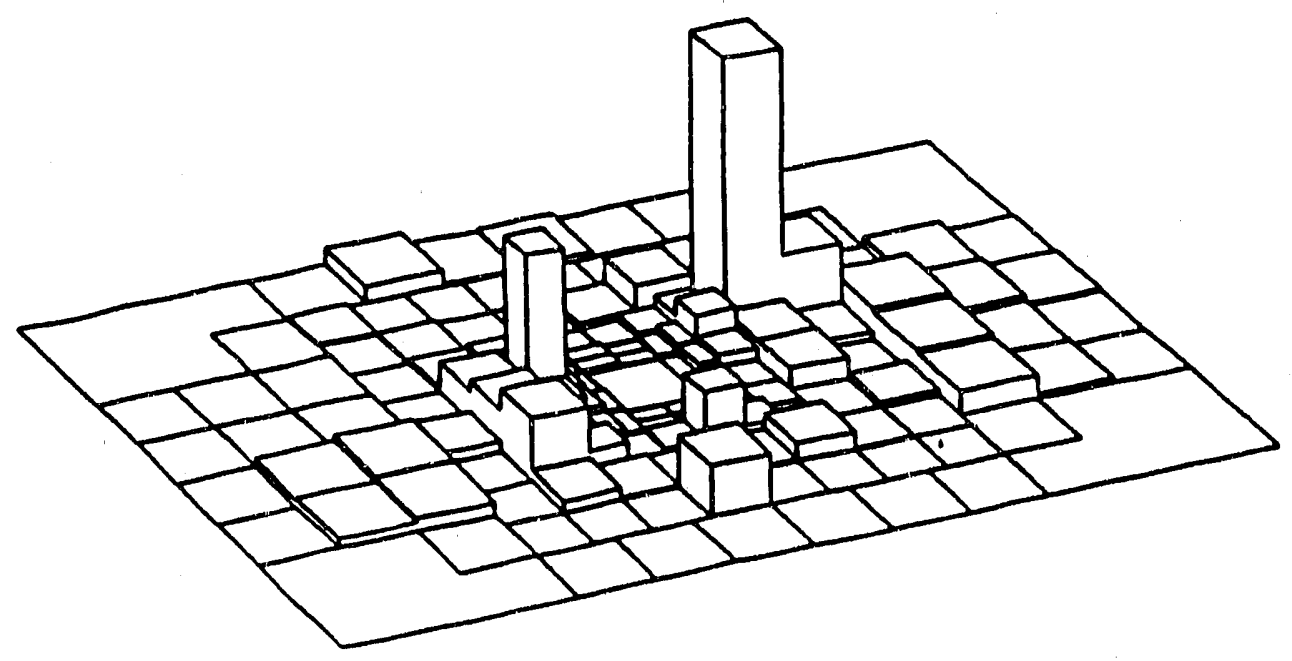

Figure 17d. Energy deposit in main calorimeter for typical event.

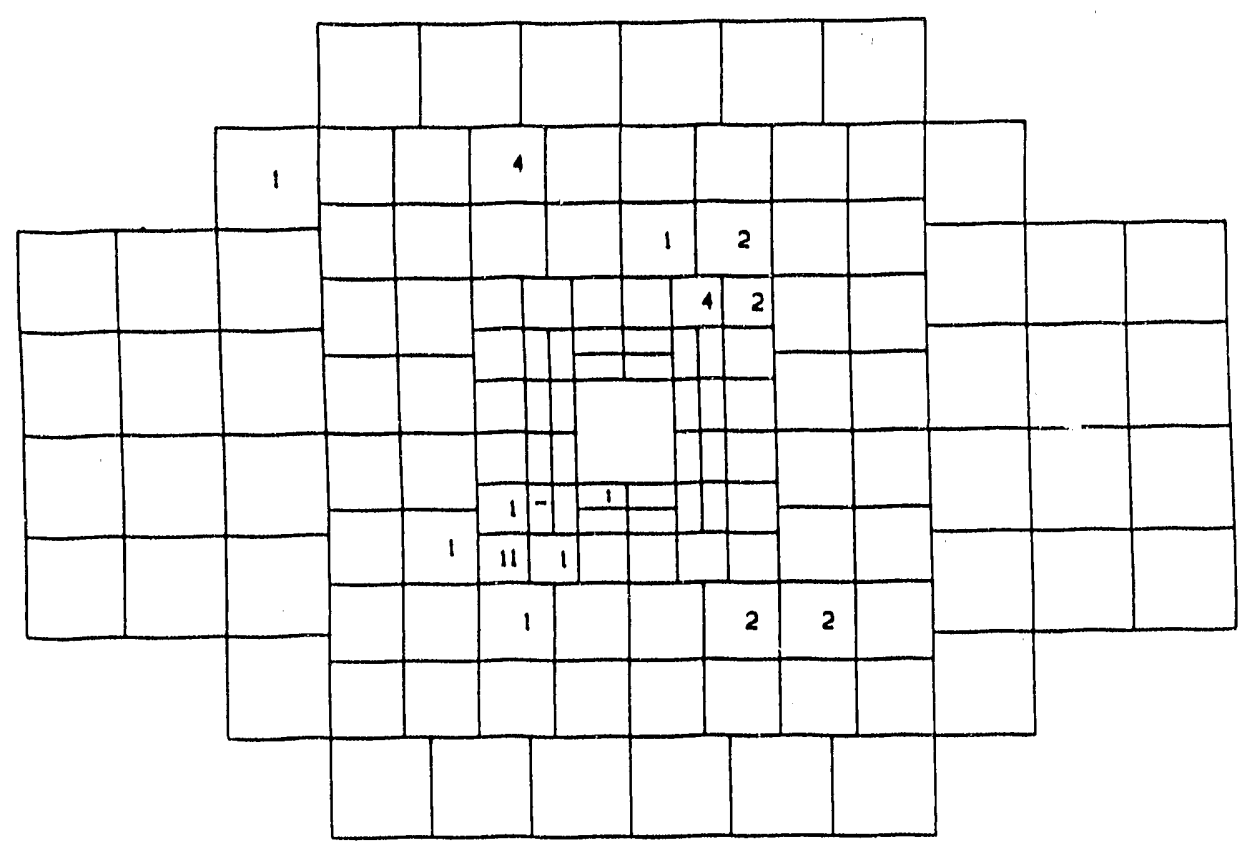

Figure 17e. Energy deposit in main calorimeter for typical event. 


\section{E683 EVENT DISPLAY}

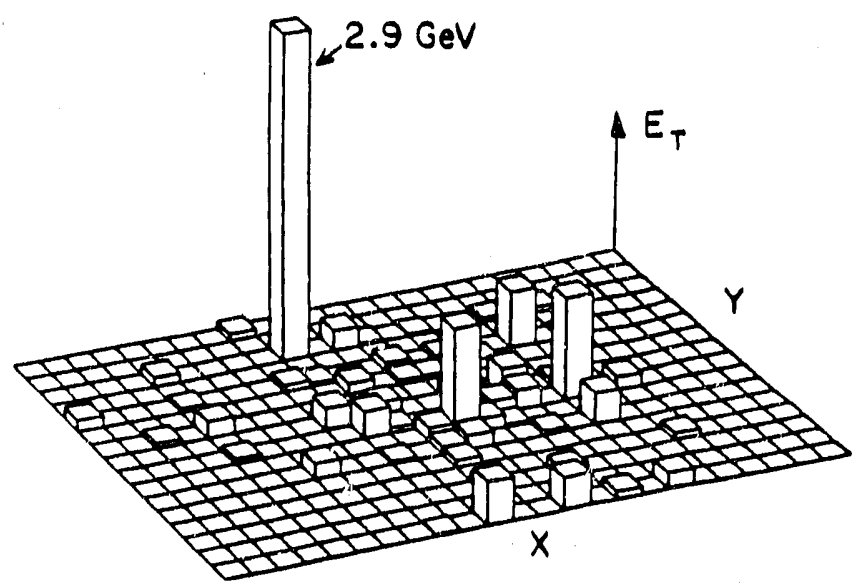

Figure 18a.

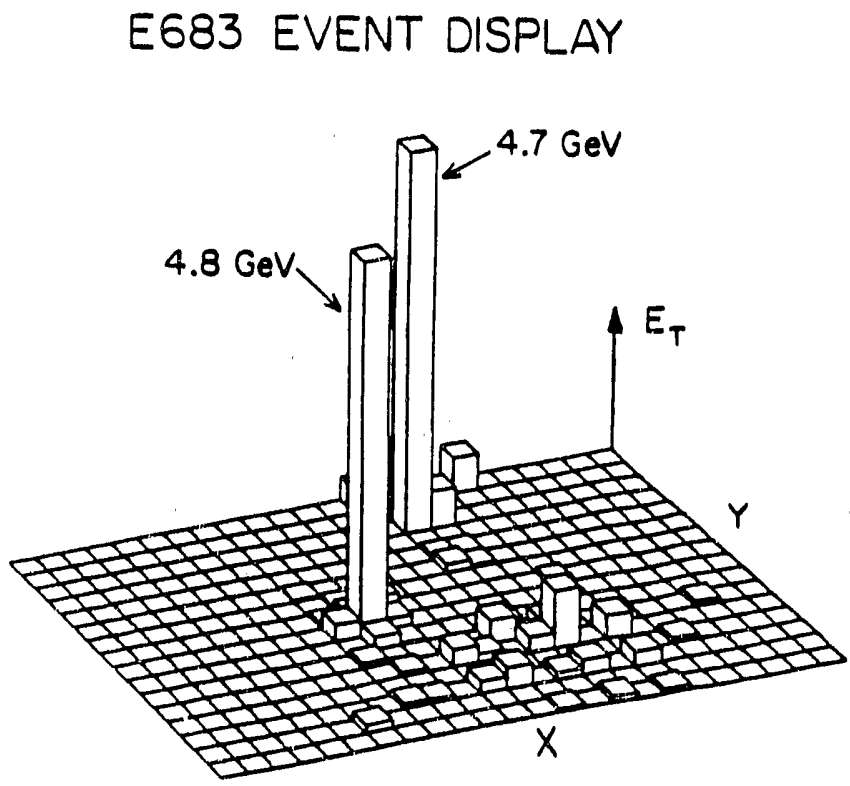

Figure $18 b$.

Figure 18. Energy deposit in main calorimeter for typical event during E683 1990 engineering run. 


\section{PART C \\ Progress Report \\ E. R. McCliment}




\section{A. Introduction}

During the past twelve months ERM and his graduate students, Timothy Dubbs (TD) and Kevin Barger (KB), have participated in several projects including

* Installation and running of experiment, E761 (Hyperon Radiative Decay);

* Preparation for the test run of experiment, E781 (Charmed Baryon Spectrometer);

* Test Run T784 (Beauty Collider Detector)

The installation of the apparatus in the Proton Center pit for experiment, E761, was accomplished in a timely manner to be ready for Session 1 of the Fermilab fixed target run. The run began on February 12, 1990 and continued until August 27, 1990. During this period tuneup and data taking were completed. All major objectives were accomplished. The Iowa silicon strip detector system performed flawlessly. Altogether 4000 tapes were written, which contain a wealth of data. Included are approximately 20,000 events of the decay $\Sigma^{+} \rightarrow$ p $\gamma$ (approximately 200 times the present world sample), 6000 events of the decay $\Xi^{-} \rightarrow \Sigma^{-} \gamma(600 \times$ world sample $)$, as well as an anti $\Sigma^{+}$signal sufficient to obtain a $10 \%$ measurement of the anti $\Sigma$ magnetic moment, and polarization data for $\Sigma^{+}$and $\Xi^{-}$. Moreover, crystal channeling was observed and it was determined that approximately 3000 channeled $\Sigma^{+}$events have been written to tape. This is a sufficient number of events to obtain a $20 \%$ measurement of the $\Sigma^{+}$magnetic moment in this manner -- enough to demonstrate the feasibility of using channeling to measure magnetic moments of other short-lived particles.

From the data on the tapes we expect to be able to measure the branching ratios for the above-mentioned radiative decays, the asymmetry parameter for $\Sigma^{+} \rightarrow$ p $\gamma$, the forward-backward asymmetry of $\Xi^{-}->\Sigma^{-} \gamma$, and the anti $\Sigma^{+}$flux, asymmetry, and magnetic moment. We also expect to obtain new measurements of the $\Sigma^{+}$magnetic moment by both channeling and conventional methods.

ERM and graduate student, TD, took part in the installation and testing of the Iowa Silicon Strip Detector system and all phases of 
data collection. Dubbs' tentative $\mathrm{PhD}$ thesis topic will be the determination of the $\Xi^{-} \rightarrow \Sigma^{-} \gamma$ branching ratio.

The installation of the silicon microstrip detector system for the E781 Charmed Baryon Spectrometer was begun near the end of the run. Some data was obtained on the performance of a single silicon strip detector plane using the latest version SVXD integrated circuit readout chip. ERM and graduate student, $\mathrm{KB}$, completed the assembly and debugging of the Iowa copies of the Berkeley SRS pattern generator and SDA fast ADC modules. As of this writing they are bench testing an SVXD chip mounted on a simplified test version of the chip carrier.

Also in connection with this experiment ERM supervised the honors project of undergraduate, Timothy Shoppa, which was to produce a Geant based Monte Carlo simulation of the E781 apparatus. This work was submitted as an Honors Thesis to the University of Iowa Department of Physics, a copy of which is included in Appendix 1 .

ERM and graduate student, KB, also participated in T784 test run (Beauty Collider Detector (BCD) development) in the Meson test beam.at Fermilab. (1) This test run consisted mainly of silicon detector studies, whose purpose

fn 1. The status of the Beauty Collider Detector is now in doubt. We nevertheless consider this test to be useful for the development of a second generation low-mass vertex detector suitable for a collider environment.

was to aid in the design of a low-mass vertex detector suitable for a collider environment. Some preliminary results of the test run are given in the preprint included in Appendix 2.

Finally, ERM with the help of graduate student, KB, has made a Monte Carlo study of $\mathrm{B}_{\mathrm{S}}-\mathrm{B}_{\mathrm{S}} \mathrm{Bar}$ mixing, using Isajet and Geant Monte Carlos. This study is nearly complete.

All work mentioned in this introduction will be described in detail below. 


\section{B. Work on Experiment E761 - Hyperon Radiative Decay}

Work on Experiment E761 includes the following:

* Magnet Scans (Tuneup)

* Beam Calibration of Neutron Calorimeter

* Shift Work

$* \Sigma^{+}$to $\Xi-$ Changeover

* Preliminary Analysis (PAW)

* Background production Monte Carlo

\section{Magnet Scans (Tuneup)}

On Feb. 1, 1990 the installation of the apparatus for the experiment in the pit of the Proton-Center area at Fermilab was virtually complete. In particular, the Iowa silicon strip detector system was in place, all cables were attached, and the detectors were in the final stages of becoming integrated into the E761 data acquisition system. Approximately 4000 channels were instrumented in three stations (Fig. 1). Also, the refurbished Fermilab neutron calorimeter, for which ERM had assumed responsibility, was installed in the data stream.

The fixed target run began on Feb. 12,1990. An initial period of approximately two months was used for tuneup - first, to steer the beam into $\mathrm{P}$ Center, then to direct the beam onto the target at various predetermined targeting angles. Fig. 2 shows a magnet scan to steer the beam vertically onto the target. This magnet scan is typical of many that were made during the initial beam-tuning phase of the experiment. In order to take advantage of bias canceling in the determination of polarization it is necessary to take data at equal and opposite targeting angles. By doing the magnet scans it was cetermined that there is an offset of $-1.0 \mathrm{deg}$ from the nominal angles calculated from the particle trajectories. Polarization increases and yield falls off with targeting angle (ref 1 -7) nominal targeting angles of +3 and $-1 \mathrm{mrad}$ (actual targeting angles $\pm 2.0 \mathrm{mrad}$ ) were chosen as a good compromise for the polarization measurements.

\section{The Sigma Configuration}

The initial configuration of the experiment (Fig. 3) was optimized for detection of the $\Sigma^{+}>\mathrm{p} \gamma$ mode. The momentum of the 
beam particle, in this case $\Sigma^{+}$, was measured by the hyperon magnets, $\mathrm{H} 1$ and $\mathrm{H} 2$, and the Iowa silicon detectors SSD1 - SSD3. The momentum of the downstream positive tracks (candidates for the decay proton) was measured by the downstream spectrometer, consisting of magnets $\mathrm{A}, \mathrm{B}$, and $\mathrm{C}$ and the proportional wire chambers, WA - WD. Photon energy was measured by the lead glass system. And the iron converter-Transition Radiation Detector (TRD) system was used to "track" the photons. Data was taken at targeting angles of +3 and $-1 \mathrm{mrad}$ as discussed above. Events written to tape were selected by a three-level hardware trigger. Level 1 is the beam trigger $\mathrm{T} 1$ defined by

$$
\mathrm{T} 1=\mathrm{B} 2 * \mathrm{~B} 3 * \text {.not.VH2*.not. } \mathrm{VH} 3,
$$

Level 2 is the photon trigger defined by

$$
\mathrm{T} 2=(\text {.not.CV1*S1).or.(.not.CV1*.not.CS1*S2) }
$$

Level 3 includes a beam rationer to exclude more than one beam particle per event:

$$
\mathrm{T} 3=\mathrm{T} 1{ }^{*} \mathrm{~T} 2 * \text { pbgsum*beam_rationer }
$$

Pbgsum demands some energy to be deposited in the lead glass system, consistent with a photon or shower produced by its conversion.

To monitor the apparatus and data acquisition a small fraction of the events (of the order of $1 \%$ ) was analyzed on-line. One measure of the goodness of our data was obtained by an "r-theta" plot. The horizontal axis ( $r$ ) is the ratio of the momentum of the supposed downstream decay baryon to the momentum of the upstream hyperon momentum. The vertical axis (theta) is a measure of the opening angle between the supposed hyperon and baryon. For a 2 body final state, $\mathrm{H} \rightarrow \mathrm{B}+$ neutral these two variables are not independent. For our case, where the lab momentum of the hyperon is large compared to the masses, $r$ and theta are related by

$$
\theta^{2} \approx\left(\frac{m_{h}}{p_{h}}\right)^{2}-\frac{m_{h}^{2}+m_{b}^{2}-m_{n}^{2}}{r p_{h}^{2}}+\left(\frac{m_{b}}{r p_{h}}\right)^{2}
$$


where $m_{h}, m_{b}$, and $m_{n}$ are the masses of incoming hyperon, baryon, and neutral decay products, respectively, and $\mathrm{ph}$ is the momentum of the incident hyperon. Thus, a 2-body decay shows up as a curved band on the r-theta plot that is similar to an inverted parabola. Fig. 4 shows an example of an $r$-theta plot. The dark band coincides with that expected from anti $\Sigma^{-} \rightarrow$ antin $\pi 0$. Similar results, obtained by using the FNAL on-line data package and an ACP farm (ten Fermilab Camac Interfaced Motcrola 68000 Computer modules), were displayed on a monitor during the run.

Approximately 1000 tapes were written over a two-month data-taking period, with of the order of $2 \times 10^{5}$ photon triggers per tape. Each tape contains a sample of prescaled (1:100) T1 triggers for normalization. The photon trigger does not distinguish $\Sigma^{+} \rightarrow p \gamma$ from the dominant decay mode $\Sigma^{+} \rightarrow p \pi^{0}$. Preliminary analysis indicates about $20 \%$ track reconstruction efficiency for $\Sigma^{+} \rightarrow$ p $\pi^{0}$ events. And we detect about 1 radiative decay/2000 $\pi^{0}$ decays, which means we have about 20,000 radiative decays on tape (about $200 \mathrm{x}$ the present world data sample!) Some runs devoted to crystal channeling measurements were interspersed with $\Sigma^{+}$data taking.

\section{Cascade Configuration}

The changeover to the cascade configuration for the reaction $\Xi-$ $\rightarrow \Sigma^{-} \gamma$ (Fig. 5) took place during a one-week period in May, 1990. This changeover was necessitated by secondary decay of the sigmadaughter to neutron $\pi^{-}$. In the cascade configuration the spectrometer magnets, $A$ and $B$, the proportional wire chambers, and the lead glass cart were moved upstream to catch the $\Sigma^{-}$daughter before a significant fraction of them decayed, and magniet $C$ was placed downstream to bend the secondary $\pi^{-1}$ s from the daughter decay away from the beam veto and neutron calorimeter. The pion veto counter was added ahead of magnet $C$ to remove pions from primary $\Sigma^{-}$decay. The beam veto counter was placed in front of the neutron calorimeter to remove undecayed beam particles from the data. The original purpose of the neutron calorimeter was to identify the neutrons from the decay of the daughter $\Sigma^{-}$. However, its main value more likely will be to separate these (good) neutrons from low energy background neutrons associated with beam-gas interactions that fool the trigger (see Section B.5 below.) 
After the changeover another two weeks were required for negative beam tuning and optimizing the trigger. The beam veto and pion veto counters were added to the photon trigger T2 described above for $\Sigma^{+}$decay. During the tuneup period ERM, graduate student, $T D$, and collaborator, Alexander Denisov, calibrated the neutron calorimeter by triggering on muons in the halo of the incident beam (trigger: $\mathrm{VH} 2 * \mathrm{VH}^{*}{ }^{*}$, not.B2*.not.B3.)

The negative beam contained only about $2 \%$ cascade, which meant that a longer running period was necessury in order to collect a significant sample of cascade radiative decays on tape. Consequently, data taking in the cascade mode continued for a 3 month period until the run ended Aug. 27. As in the case of $\Sigma^{+}$decay a small number of runs devoted to crystal channeling were interspersed with the cascade data taking. ERM and graduate student, TD, were involved in all phases of the data taking.

\section{B.4. Preliminary Search for Radiative Cascade- Decays}

ERM attempted to find the $\Xi^{-} \rightarrow \Sigma^{-} \gamma$ signal by means of a suggestion originally due to $A$. Denisov. The idea is to extrapolate the downstream charged track ("baryon") to the beam veto counter. Monte Carlo studies show that if this track is a $\Sigma^{-}$daughter from a $\Xi^{-}$ radiative decay, the momentum direction of the resulting neutron is strongly correlated with it. Thus, charged tracks which extrapolate to the beam veto (which would be vetoed if they actually made it to this counter) are likely to be candidates for radiative decilys. These tracks were selected by an off-line program and examined by means of the CERN PAW software package, which enables one to make histograms, put in cuts, and examine correlations between variables interactive!y.

Fig. 6 a through $c$ show resulting histograms for the neutral mass-squared based on the hypothesis, $\Xi^{-}>\Sigma^{-} \gamma$, as a series of cuts was applied to the data. In Fig. 6a there are no cuts other than a loose requirement that the $x$ position of the extrapolated tracks fall within $2.0 \mathrm{~cm}$ of the beam veto. The latch selection (Fig. 6b) requires that there be energy in the lead glass (latch 13), energy in the neutron calorimeter (latch 9), and no hit in the beam veto counter (.not.latch 15). The full set of cuts includes (Fig. 6c) a chi-square $<=$ 3.0 on the tracks used, $>=0.2 \mathrm{GeV}$ in the lead glass blocks pointed to by the extrapolated neutral track, $0.5<\mathrm{E} / \mathrm{p}<1.5$, a requirement that the extrapolated charged track be within the beam veto, and a mass 
cut to remove anti $\Sigma$ 's and kaons. The resulting mass-squared distribution shows a peak near 0. But the width and the slightly negative position of the peak indicates that this may be background rather the sought after signal. Fig. 7 is an accompanying $r$-theta plot of the surviving events. This plot is not very convincing. However, only a small fraction of the available events were used in this search. Work is now in progress to enlarge the scope of this analysis and use a much larger event sample. The appearance of the appropriate parabola on the $r$-theta plot would definitely prove that a sample of $\Xi^{-} \rightarrow \Sigma^{-} \gamma$ events has been found. (fn2)

fn2. C. Newsom has evidence of a mass peak by a different technique, namely by dividing the events according to their vertex positions and exploiting the lifetime of the Cascade-. Newsom's method is useful for a branching ratio measurement but has no hope for asymmetry determination.

\section{B.5. Background Monte Carlo Studies}

Because the incident beam is only $2 \%$ cascade, and the radiative decay branching ratio is of order $1.0 \times 10^{-3}$, the signal to background ratio at best is of order $2 \times 10^{-5}$. Therefore, any background present at this same level is important. It is necessary to investigate all such backgrounds with an eye to eliminating them or at least reducing them to a small fraction of the desired cascade radiative decay mode. ERM has begun a systematic Monte Carlo study of backgrounds arising from beam-gas interactions with the dominant $\pi^{-}$component of the beam. The importance of this background has become apparent from what appears to be a large number of beam tracks seen on-line despite the trigger requirement that photons be present in an accepted event.

To carry out the study an adaptation of the LUND MONTE CARLO (PYTHIA + JETSET) was used to produce a PAW file similar to those obtained with real data. At present an exploratory study of the reaction

$$
\pi^{-}+\mathrm{n} \rightarrow \pi^{-}+\mathrm{n}+\pi^{0}+\mathrm{X}
$$

is underway. Histograms of some results obtained using the interactive PAW program are shown in Fig. 8a,b. We assume that these events "fake" radiative cascade decay, by making the 
hypothesis that the incident $\pi^{-}$is a fake $\Xi^{-}$, and the highest energy $\pi^{-}$is a fake $\Sigma^{-}$, then calculate the mass-squared of the "neutral particle" based on this hypothesis. Fig. 8 a is a histogram of this quantity with no cuts applied except for the requirement of at least one $\pi^{0}$, which coincides with the trigger requirement of electromagnetic energy in the event. Cuts are then put in 10 make these fake ovents more closely resemble radiative cascade decay. First, a cut is made to eliminate all charged $\pi$ 's with energy less than about $20 \mathrm{GeV}$ as these would likely be swept away by the analyzing magnets (Fig. 8b). These events were also required to have gamma energy below $90 \mathrm{GeV}$ corresponding to an upper kinematic limit of the gamma energy of $75 \mathrm{GeV}$ in cascade to sigma gamma decay. We see from this histogram that such events do fake the signal. Fig. 9 shows an $r$-theta plot of these events, from which it is apparent that they fall in the allowed region for $\Xi^{-} \rightarrow \Sigma^{-} \gamma$ decay. However, if we look at the neutron spectrum (Fig. 10) we see that most of these events have only a low energy neutron. This suggests that we can reduce this background by cutting on the energy in the neutron calorimeter. In the real data a cut of $40 \mathrm{GeV}$ is made on a latch bit for the neutron calorimeter. We find that we can eliminate $96 \%$ of the fake events by requiring the neutron energy to be above $4.0 \mathrm{GeV}$. Fig. 11 is an r-theta plot with the neutron energy cut in place. Comparison with Fig. 9 clearly shows this reduction. The conclusion from this exploratory study is that useful insights can be obtained. about backgrounds and their elimination. Further study is contemplated.

\section{Work on BCD Silicon Detector Test Run}

ERM along with graduate student, $K B$, took part in a test of single and double sided $A C$ coupled silicon microstrip detectors wire bonded to the Berkeley SVXD integrated readout chip. The original intent of this test run was to aid in the design of a silicon vertex detector for the proposed Beautiful Collider Detector (BCD). The status of this detector is now uncertain, but the need for a sophisticated vertex detector to do $B$ physics in a collider environment has not diminished. This project can now be thought of more as generic detector development.

The detectors were placed in the test stand designed by $C$. Lindenmeyer and built by Iowa (Fig. 12). The test stand was designed to be able to rotate the plane of alternate detectors so that nonnormal incidence can be studied. In this study we used four 
detectors configured as a set of hodoscopes placed roughly 8 inches apart along the Meson Test beam at Fermilab. The beam had a momentum of $275 \mathrm{GeV} / \mathrm{c}$ and consisted mainly of negative pions. We succeeded in reading out the detectors in latch-all mode, we were able to do tracking in one view; and we measured the resolution of the detectors as a function of angle of incidence. The accompanying preprint in Appendix 2 describes the results in detail. Briefly, we found that our measured resolutions $-10.4 \mu \mathrm{m}$ at normal incidence to $30.5 \mu \mathrm{m}$ at $45^{0}$ incidence are within $10 \%$ of the values predicted by the Monte Carlo (see ref 8 included as Appendix B, Fig. 9).

\section{Work on $B_{\underline{S}}-\underline{B}_{\underline{S}} \underline{B}$ ar Mixing}

ERM along with graduate student, $\mathrm{KB}$, has also been working on a simulation of $\mathrm{B}_{\mathrm{S}}-\mathrm{B}_{\mathrm{S}} \mathrm{B}$ ar mixing with an eye to understanding what kind of vertex resolution is needed to observe it. $B_{S}-B_{S} B$ ar mixing is important to study because it can provide a crucial test of the standard model, which restricts the mixing parameter, $\mathrm{x}_{\mathrm{S}}$ (the ratio of the mixing frequency to the lifetime of $B_{S}$ ) to a value greater than 3.0. The work described here is based on the Isajet particle generator with Gant simulation of the decays in a simple spherical geometry. Our own modifications of Geant produce the mixing based on the mixing formulas,

$$
\begin{aligned}
& N_{B} \propto e^{-t / \tau}\left[1+\cos \left(x_{s} t / \tau\right)\right] \\
& N_{\text {BBar }} \propto e^{-t / \tau}\left[1-\cos \left(x_{s} t / \tau\right)\right]
\end{aligned}
$$

This work is not yet ready for publication, but some preliminary resul." are given here.

The point of this exercise is to assess the minimum number of reconstructed events needed in order to see $\mathrm{B}_{\mathrm{S}}-\mathrm{B}_{\mathrm{S}} \mathrm{Bar}$ mixing. In order to see things more clearly one of the $B_{S}$ 's is required to have a nonleptonic decay, while the other is allowed only leptonic decay modes. The idea is that in order to sbserve mixing the trigger would have to identify both the $B_{S}$ and its $B B a r$ partner (or vice versa). One of the B's could be identified by a leptonic decay and the other would have to be reconstructed in order to distinguish $B_{S}$ from $B^{0}$. At present this study generates B-BBar events in a collider environment 
at $2 \mathrm{TeV}$ (Fermilab energy) and $40 \mathrm{TeV}$ (SSC energy). Fig. 13a and $\mathrm{b}$ shows the generated lifetime distribution of $B_{S}$ 's and $B_{S} B$ ars in their own rest frame, starting with pure $B_{s}$ 's at the production vertex.

Once the mixing has taken place the strategy is to mimic experiment by allowing the B's and their offspring to decay, then try to identify the B's and BBars from their decay products. To simplify the study this is done by "triggering" on a high-energy lepton of a given sign. The Monte Carlo, of course, has the advantage that one can remember whether the original $\mathrm{B}$ has mixed or not.

Consequently, the leptons can be classified as "right sign" leptons or "wrong sign" leptons based upon whether the whether the original $B_{S}$ ( $\mathrm{B}_{\mathrm{S}} \mathrm{Bar}$ ) has changed identity or not. Thus, if there were no mixing there would be no wrong sign leptons coming from the B's themselves. All such leptons would then come from the decay products -- mainly $D$ mesons.

This provides a useful procedure to set the lepton energy cut. First, the mixing is turned off. Then the energy of the cut is raised until there is no change in the fraction of right-sign to wrong-sign leptons (wrong sign leptons here come only from daughter decays). At this point one has optimum signal to background discrimination. We find that the higher the collision energy the less the discrimination provided by the sign of the leptons. At $2 \mathrm{TeV}$ the maxinum ratio of right-sign to wrong-sign leptons is $2: 1$ with an optimum lepton energy cut of $10 . \mathrm{GeV}$, whereas at $40 \mathrm{TeV}$ this ratio decreases to $1.6: 1$ with optimum lepton energy cut of $25.0 \mathrm{GeV}$.

Once the value of the energy cut has been established the "experimental" mixing can be studied as a function of the mixing parameter, $x_{s}$. (fn4) The mixing signal is selected by the sign of the lepton above the energy cut.

fn 4. A small $\mathrm{pt}$ was also put in. The results are not too sensitive to the $p_{t}$ cut so this was put to a low value of $100 \mathrm{MeV}$ because a higher value greatly reduces the mixing signal for a given number of events generated -- here 30,000 .

Other things being equal the mixing signal is sensitive to the experimental vertex resolution and to the experimental determination of the energy of the $B_{S}\left(B_{S} B a r\right)$. The former smears the distance the $\mathrm{B}$ travels in the lab before decaying, and the latter smears the transformation to the rest frame of the B. Both of these resolutions smizars the iifetime determination in the center-of-mass. 
Fig. $14 \mathrm{a}$ and $\mathrm{b}$ show typical histograms of the $\mathrm{B}_{\mathrm{S}}$ lifetimes obtained from of the right-sign and wrong-sign leptons. As one would expect it becomes more difficult to detect mixing as the oscillation frequency increases. If the mixing parameter turns out to be about 5.0 it should be relatively easy to observe with vertex smearing of $10 \%$ of nominal $c \tau$ and $10 \%$ smearing of the B's energy (Gaussian smearing assumed).

ERM has determined ciat ti.e sensitivity of the data cinii be increased by combining the right sign and wrong sign histograms. The itea here is that the phase of the oscillation is correlated in the two histograms, whereas the background is not. Moreover, the right sign ossillations stari out at maximum, corresponding to the fact that a pure B (or BBar) state was created at the production vertex. This also means the wrong sign oscillations start at zero. Therefore, there is a phase difference of $180^{\circ}$, which means that if the two histograms are subtracted the signal to background ratio will improve. Fig. 15 shows a typica! resultant histogram, in this case with the mixing parameter, $\mathrm{x}_{\mathrm{S}}=5.0,10 \%$ smearing of the vertex, and $10 \%$ smearing of the $\mathrm{B}_{\mathrm{S}}$ lab energy.. This procedure substantially enhances the oscillations relative to the background.

To be more quantitative fits of the leptonically selected distributions were made to the theoretical B-lifetime formulas above. Fig. 16a-c are represcntative iistograms for the $2.0 \mathrm{TeV}$ case. The results suggest that for comparable errors at least 10 times the statistics are needed to measure the $\mathrm{B}_{\mathrm{S}}-\mathrm{B}_{\mathrm{S}} \mathrm{Bar}$ mixing parameter if it is near 20.0 than if it is near 5.0. The results are similar for the 40.0 $\mathrm{TeV}$ case.

So far backgrounds other than leptons from daughter derays have not been considered, nor have effects from $\mathrm{K}-\pi$ misidentification. These, of course, are important also.

\section{E. Work on the E781 Monte Carlo}

ERM and undergraduate student, Timothy Shoppa, have constructed a Geant-based Monte Carlo of the E781 Selex Charned Baryon Spectrometer. This work was a senior honors project for $\mathrm{Mr}$. Shoppa and resulted in an Honors thesis. An abridged copy of Mr. Shoppa's thesis (left out is an appendix containing the Fortran source code) is included as Appendix A. So far we have installed the detector geometry and have included a crude particle generator based on a simple Feynman $x$ and $p_{t}$ distribution for charmed baryons (the standard particle generators, Pythia and Isajet, do not 
have provision for hyperon collisions.) This detector is to have a segmented target. so we have included random production from each of the target segments. For more details the reader is referred to Mr. Shoppa's honors thesis. This Monte Carlo is the starting point for serious studies of backgrounds and optimization of the detector geometry. ERM and graduate student, $\mathrm{KB}$, intend to carry on with these studies.

\section{F. Work on E781 Hardware}

Work is progressing on construction and testing of l'ardware for experiment E781. However, delays in obtaining equipment from the manufacturers and precedence given to the running of E761 drastically curtailed the E781 test run at Fermilab during the first session of the run. However, we expect to continue the test run in the second fall-winter session scheduled to begin in January, 1991. Our intention is to run parasitically with E800 in P-Center, and the hardware is now being installed in the pit downstream of the E800 apparatus. To date the only devices that have had any beam testing whatsoever are the ring imaging Cherenkov detector (RICH) and a single plane of the silicon detector system.

The RICH group has constructed a test version of the RICH detector with just enough phototubes to test the pattern recognition capabilities of the device. Some preliminary studies have been carried out in the beam.

The silicon detectors have been ordered but the delivery has been slow. To date four $2 \mathrm{~cm} \times 2 \mathrm{~cm}$ detectors and $25 \mathrm{~cm} \times 5 \mathrm{~cm}$ detectors have been mounted on their chip carriers and wire bonded to SVXD chips out of a total of 20 ordered have been delivered. Of these only one $5 \times 5$ detector has been beam tested so that no tracking information is as yet available. Iowa is responsible for 102 $\mathrm{x} 2$ detectors. These are single-sided DC coupled devices

manufactured by Hamamatsu. Each has 1000 strips with $20 \mu \mathrm{m}$ pitch. Readout is achieved by means of the Berkeley SVXD readout chip that is controlled and read by Berkeley SRS pattern generator and SDA flash ADC modules. At Iowa we have acquired, tested, and debugged one each of these modules. But we do not as yet have a fully bonded detector in our possession. However, we do have an SVXD chip that has been wire bonded to a simple chip carrier and is suitable for test purposes. We have just begun bench tests of this chip. When we are satisfied with the results of these tests we will proceed to test a full detector with the aid of a radioactive source. 
Fig. 17 shows a diagram of a chip carrier with a microstrip detector and its complement of SVX chips aboard. Fig. 18 shows a spectrum obtained from the one working detector in the test beam. This spectrum indicates that the pattern generntor, the SVX chip and the data acquisition system are working correctly -- a good start, but much work yet to be done.

\section{G. Summary}

ERM and his students have worked on several projects during Proposal year 90-91. Foremost was the running of the E761 experiment at FNAL (with graduate student, Timothy Dubbs.) Other projects included a Monte Carlo study of pion production background in this experiment, work on a Geant-based SELEX Monte Carlo (with undergraduate student Timothy Shoppa, which lead to Shoppa's senior honors thesis), the E784 (BCD) test run of an AC-coupled silicon detector system with the Berkeley SVX-chip readout (with graduate student, Kevin Barger), Monte Carlo studies of B-BBar mixing with graduate student, Kevin Barger, and work on hardware for the E781 silicon microstrip detector system (with graduate student, Kevin Barger.). 
H. Expenditures

Through $9 / 30 / 90$

\begin{tabular}{lr}
\hline Labor & $\$ 25,880$ \\
Staff Benefits & 2793 \\
Travel (Domestic - includes summer at FNAL) & 4378 \\
Materials & 787 \\
Services & 2398 \\
Phone & 680 \\
Freight & 24 \\
Equipment Rental & 48 \\
Indirect Costs & 12721 \\
\hline & \\
Total & $\$ 49709$ \\
Projected through $1 / 31 / 91$ & \\
& \\
\hline Labor & $\$ 5328$ \\
Staff Benefits & 280 \\
Travel & 1500 \\
Indirect costs & 2985 \\
Total & $\$ 10093$ \\
Grand Total & $\$ 59802$
\end{tabular}


1. J. L. THORN, T. R. CARDELLO, P. S. COOPER, L. J. TEIG, Y. W. WAH, C. ANKENBRANDT, J. P. BERGE, A. E. BRENNER, J. BUTLER, K. DOROBA, J. E. ELIAS, J. LACH, P. LAURIKAINEN, J. MaCLACHLA.N, J. MARRINER, E. W. ANDERSON, A. BREAKSTONE, E. I. ROSENBERG, and E. MCCLIMENT

Search for Heavy Charged Particles and Light Nuclei and Antinuclei Produced at High Energies

Phys. Rev. D 31, 451-463, 1985

2. T. R. CARDELLO, P. S. COOPER, L. J. TEIG, J. L. THORN, Y. WAH, C. ANKENBRANDT, J. P. BERGE, A. E. BRENNER, J. BUTLER, K. DOROBA, J. E. ELLAS, J. LACH, P. LAURIKAINEN, J. MaCLACHLAN, J. MARRINER, E. W. ANDERSON, A. BREAKSTONE, E. I. ROSENBERG, and E. McCLIMENT Charged-Hyperon Production by $400 \mathrm{GeV}$ Protons Phys. Rev. D 32, 1-10, 1985

3. S. Y. HSUEH, D. MÜLLER, J. TANG, R. WINSTON, G, ZAPALAC, E. C. SWALLOW, J. P. BERGE, A. E. BRENNER, P. GRAFSTRÖM, E. JASTRZEMBSKI, J. LACH, J. MARRINER, R. RAJA, V. J. SMITH, E. MCCLIMENT, C. NEWSOM, E. W. ANDERSON, A. S. DENISOV, V. T. GRACHEV, A. V. KULIKOV, V. A. SCHEGELSKY, D. M. SELIVERSTOV, N. N. SMIRNOV, N. K. TERENTYEV, I. I. TKATCH, A. A. VOROBYOV, P. S. COOPER, P. RAZIS, and L. J. TEIG Measurement of the Electron Asymmetry in the Beta Decay of Polarized $\Sigma^{-}$Hyperons Phys. Rev. Lett. 54, 2399-2402, 1985

4. Y. W. WAH, T. R. CARDELLO, P. S. COOPER, L. J. TEIG, J. L. THOR.N. C. ANKENBRANDT, J. P. BERGE, A. E. BRENNER, J. BUTLER, K. DOROBA, J. ELIAS, J. LACH, P. LAURIKAINEN, J. MacLACHLAN, J. MARRINER, E. W. ANDERSON, A. BREAKSTONE, and E. MCCLIMENT

Measurement of $\Sigma^{-}$Production Polarization and Magnetic Moment Phys. Rev. Lett. 55, 2251-2255, 1985

5. G. ZAPALAC, S. Y. HSUEH, D. MÜLLER, J. TANG, R. WINSTON, E. C. SWALLOW, J. P. BERGE, A. E. BRENNER, P. GRAFSTRÖM, E. JASTRZEMBSKI, J. LACH, J. MARRINER, R. RAJA, V. J. SMITH, E. MCCLIMENT, C. NEWSOM, E. W. ANDERSON, A. S. DENISOV, 
V. T. GRACHEV, V. A. SCHEGELSKY, D. M. SELIVERSTOV, N. N. SMIRNOV, N. K. TERENTYEV, I. I. TKATCH,

A. A. VOROBYOV, P. S. COOPER, P. RAZIS, and L. J. TEIG

Measurement of the $\Sigma^{-}$Magnetic Moment Using the $\Sigma^{-} \rightarrow n e^{-} \bar{\nu}$

and $\Sigma^{-} \rightarrow \mathrm{n} \pi^{-}$Decay Modes

Phys. Rev. Lett. 57, 1526-1529, 1986

6. S. Y. HSUEH, D. MÜLLER, J. TANG, R. WINSTON, G. ZAPALAC,

E. C. SWALLOW, J. P. BERGE, A. E. BRENNER, P. S. COOPER,

P. GRAFSTRÖM, E. JASTRZEMBSKI, J. LACH, J. MARRINER, R. RAJA,

V. J. SMITH, E. MCCLIMENT, C. NEWSOM, E. W. ANDERSON, A. S.

DENISOV, V. T. GRACHEV, V. A. SCHEGELSKY, D. M. SELIVERSTOV,

N. N. SMIRNOV, N. K. TERENTYEV, I. I. TKATCH, A. A. VOROBYOV,

P. RAZIS, and L. J. TEIG

High.Precision Measurement of Polarized- $\Sigma^{-}$Beta Decay

Phys. Rev. D 38, 2056-2076, 1988

7. L. H. TROST, E. R. MCCLIMENT, C. R. NEWSOM, S. Y. HSUEH,

D. MÜLLER, J. TANG, R. WINSTON, G. ZAPALAC, E. C. SWALLOW,

J. P. BERGE, A. E. BRENNER, P. S. COOPER, P. GRAFSTRÖM,

E. JASTRZEMBSKI, J. LACH, J. MARRINER, R. RAJA, V. J. S.MITH,

E. W. ANDERSON, A. S. DENISOV, V. T. GRACHEV, V. A. SCHEGELSKY',

D. M. SELIVERSTOV, N. N. SMIRNOV, N. K. TERENTYEV, I. I. TKATCH,

A. A. VOROBYOV, P. RAZIS, and L. J. TEIG

New Measurement of the Production Polarization and Magnetic Moment of the $\Xi-$ Hyperon

Phys. Rev. D 40, 1703-1707, 1989

8. M. LAMBRECHT, H. ATTIAS, K. BARGER, G. KALBFLEISCH,

D. KAPLAN, P. KARCHIN, J. KUEHLER, E. MCCLIMENT,

W. ROSS, J. SINNOT, P. SKUBIC, S. UTKU

Buam Tests of Silicon Microstrip Detectors with VLSI

Readout

Submitted to Transactions of the JEEE, Oct. 1990. 


\section{E761}

\section{Sillcon Microstrip Detector}

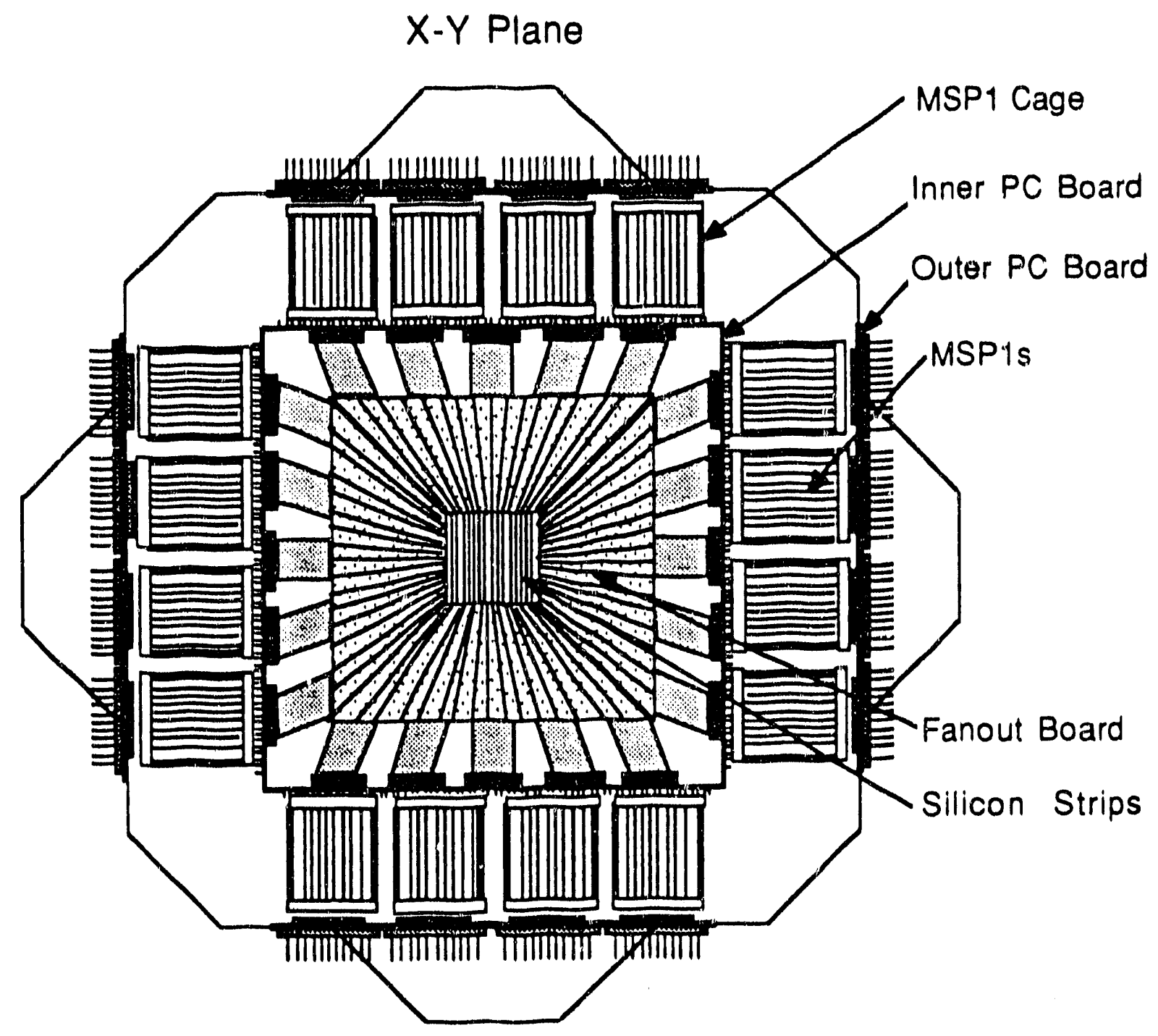

Figure 1. An Iowa Silicon Microstrip Detector. 


\section{Vertical Scan to Find Target}

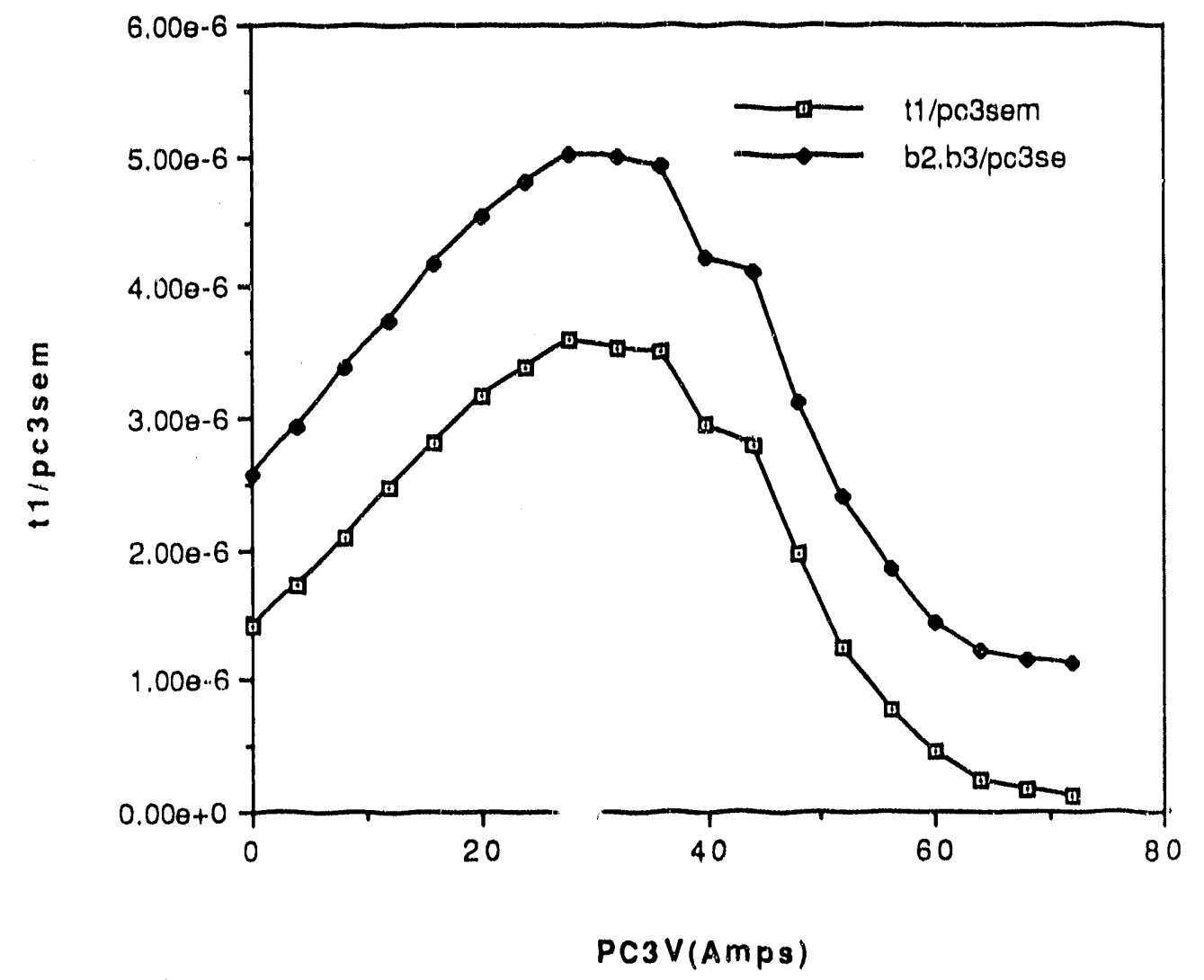

Figure 2. A sample magnet scan. The horizontal axis is the magnet current of upstream vertical stearing magnet PC $3 \mathrm{~V}$. The vertical axis is the number of counts in the beam counter $\mathrm{T} 1$ normalized to the proton flux measured by special wire chamber PC3SEM. 

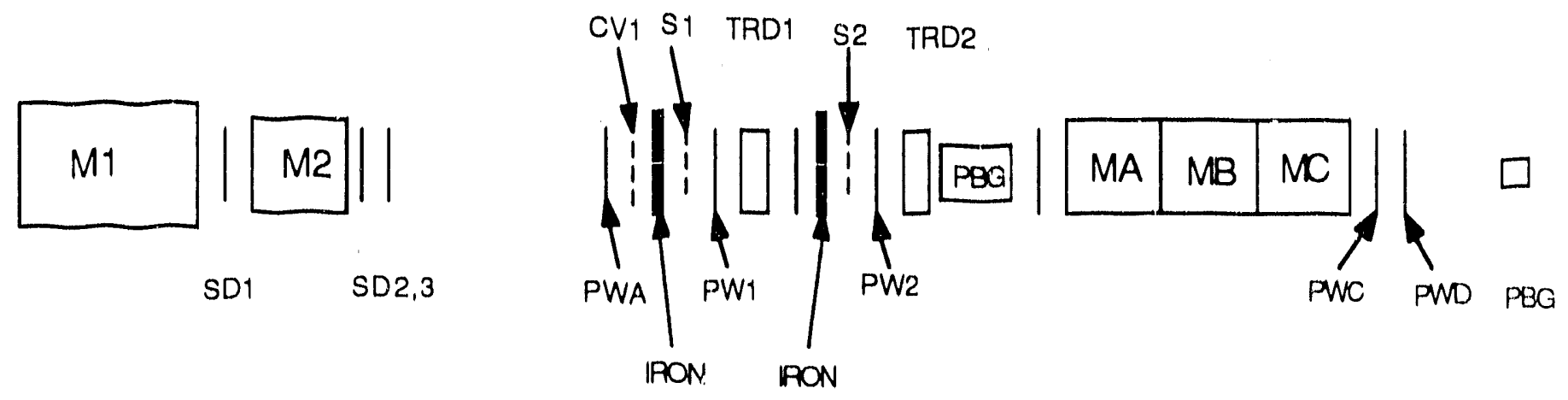

Figure 3. The experimental configuration for detection of $\Sigma \rightarrow p \gamma$. The distances are not to scale. 


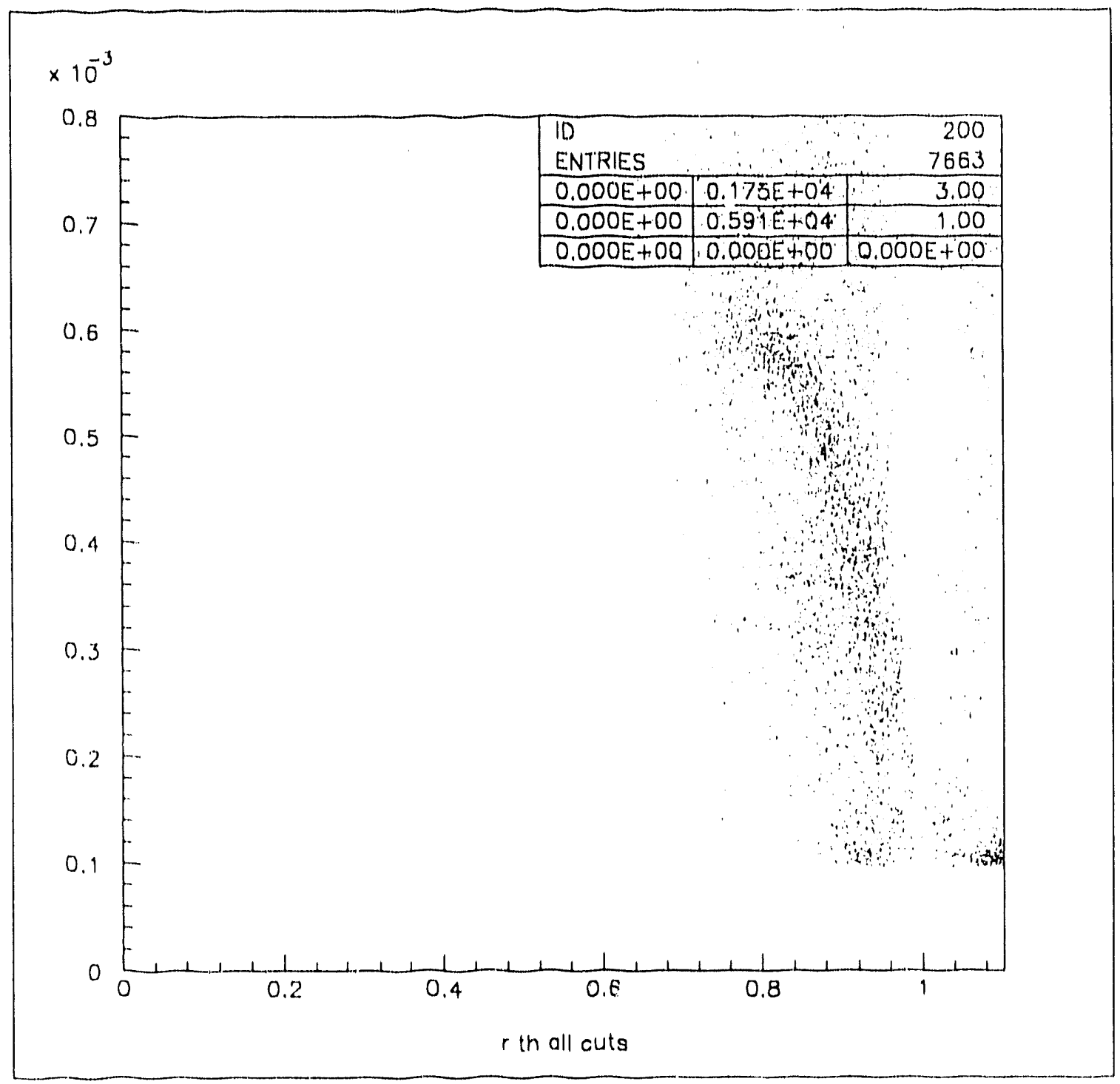

Figure 4. An $r$-theta plot. This plot is from cascade data. The shaded band matches the decay anti $\Sigma^{-} \rightarrow$ pbar $\pi^{0}$. 

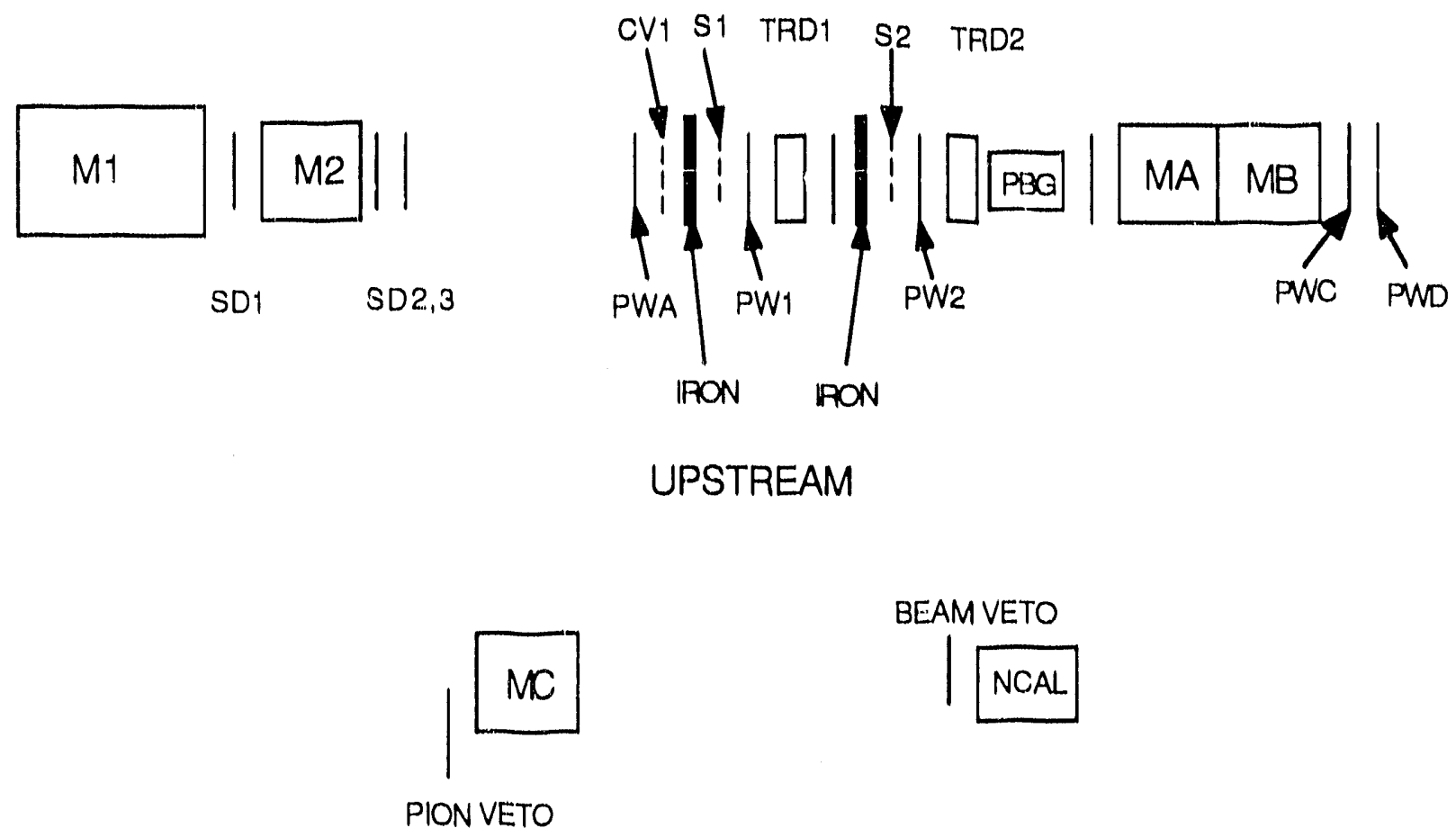

DOWNSTREAM

Figure 5. The experimental configuration for detection of $\Xi^{-} \rightarrow \Sigma^{-} \gamma$. The distances are not to scale. 


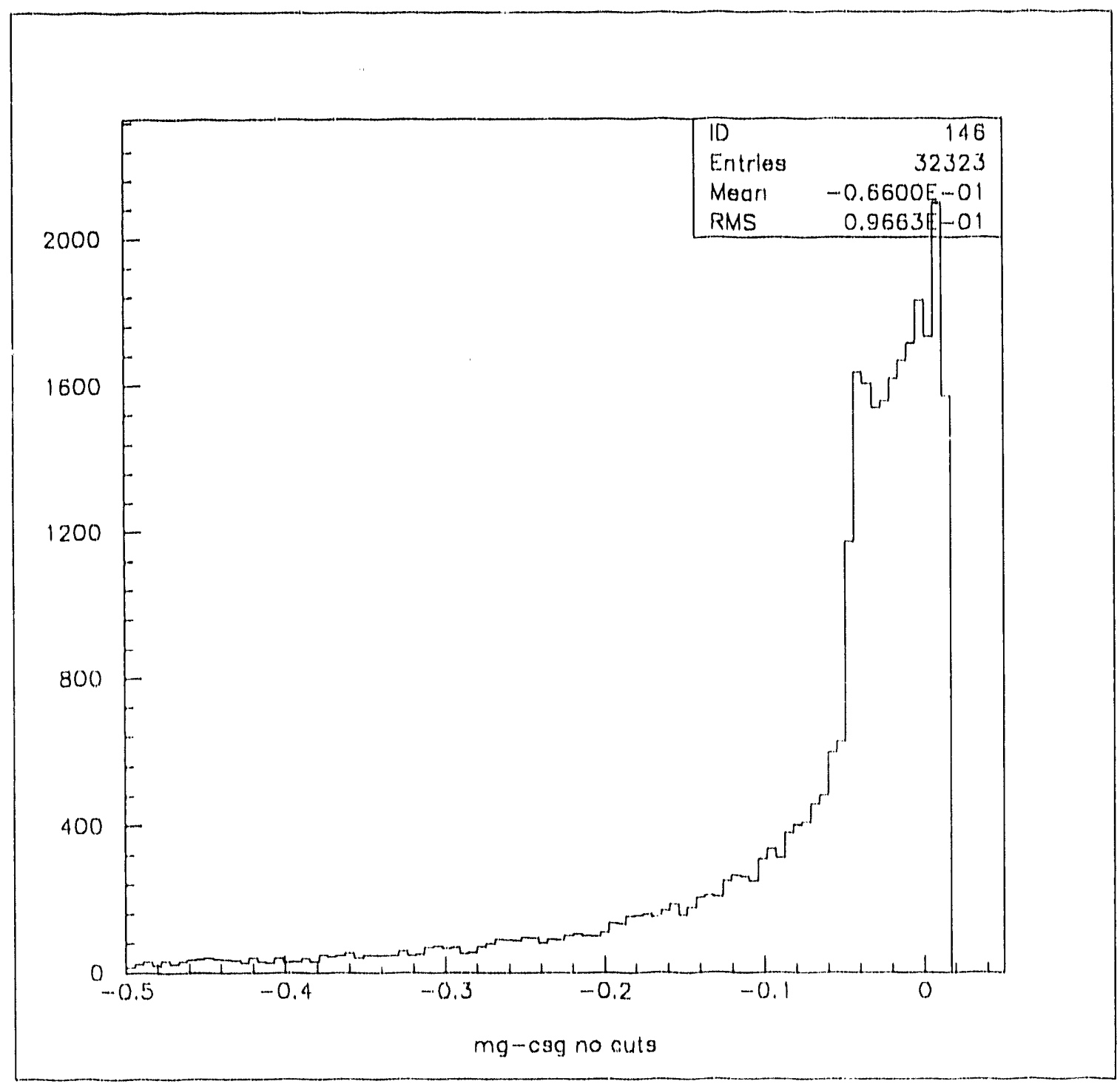

Figure $6 a$ Histogram of the mass-squared of a neutral based on the hypothesis $\Xi-\rightarrow>-\gamma$. a. No cuts. h, Latch selection requiring energy in the neutron calorimeter (9) and lead glass (13), and no signal in the neutron veto counter (n15), c. Latch selection as in $b$, and chisquare, local lead glass energy, $E$ over $P$, and mass cuts. 


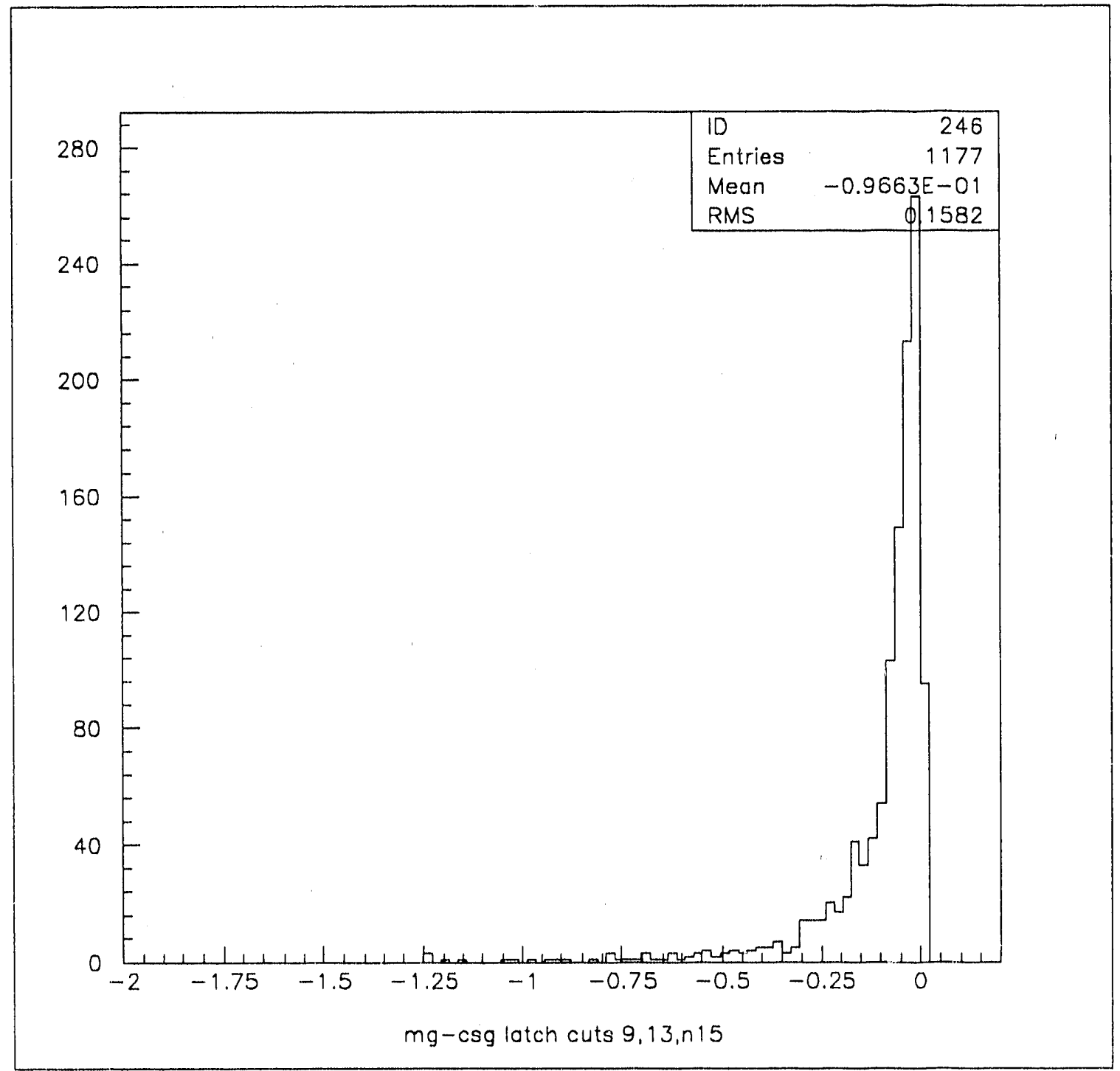

Figure $6 b$ Histogram of the mass-squared of a neutral based on the hypothesis $\Xi^{-} \rightarrow \Sigma^{-} \gamma$. a. No cuts. b. Latch selection requiring energy in the neutron calorimeter (9) and lead glass (13), and no signal in the neutron veto counter $(n 15)$. c. Latch selection as in $b$, and chisquare, local lead glass energy, $E$ over $P$, and mass cuts. 


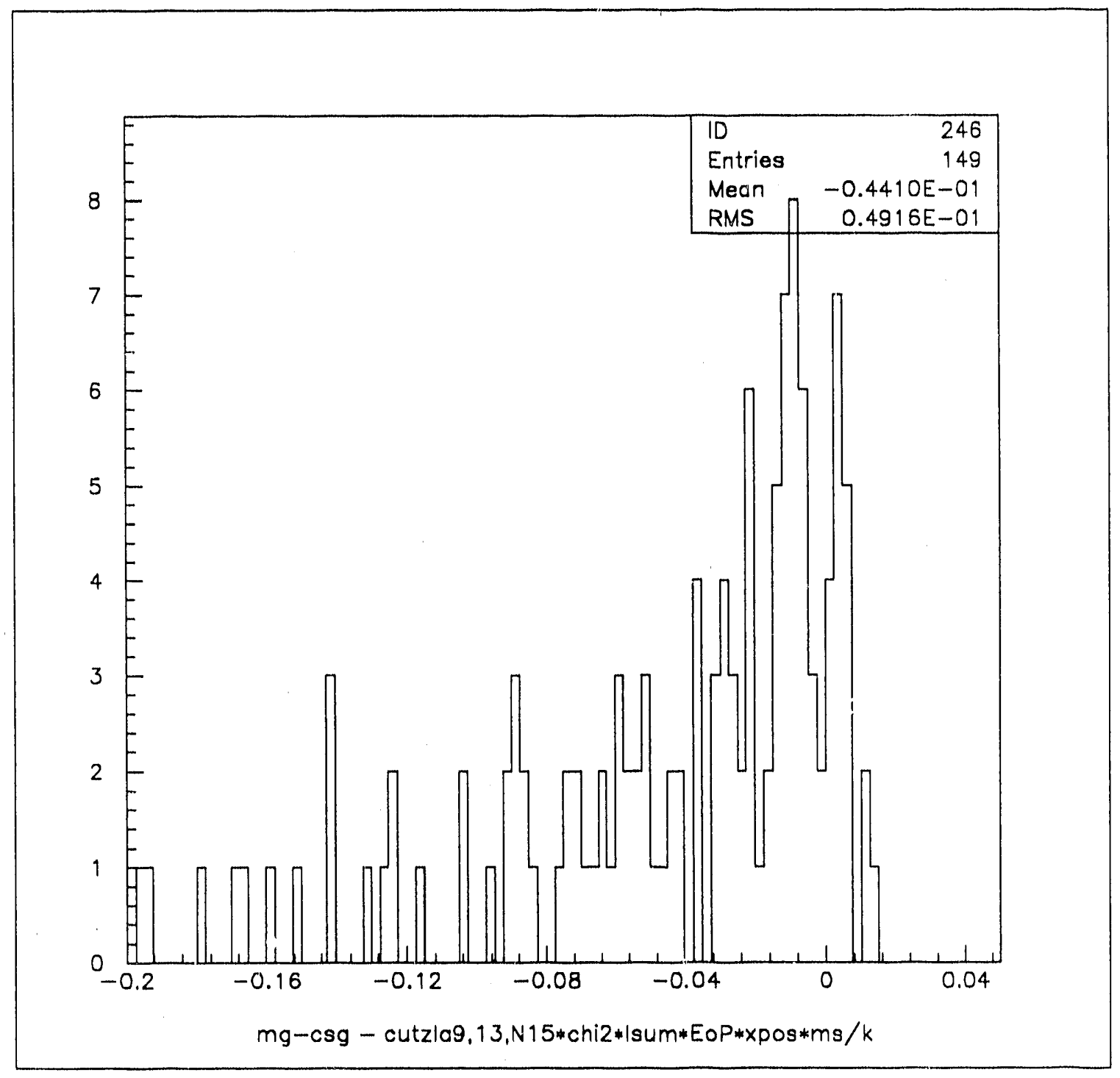

Figure 6c Histogram of the mass-squared of a neutral based on the hypothesis $\Xi^{-} \rightarrow \Sigma^{-} \gamma$. a. No cuts. b. Latch selection requiring energy in the neutron calorimeter (9) and lead glass (13), and no signal in the neutron veto counter (n15). c. Latch selection as in $b$, and chisquare, local lead glass energy, E over $P$, and mass cuts. 


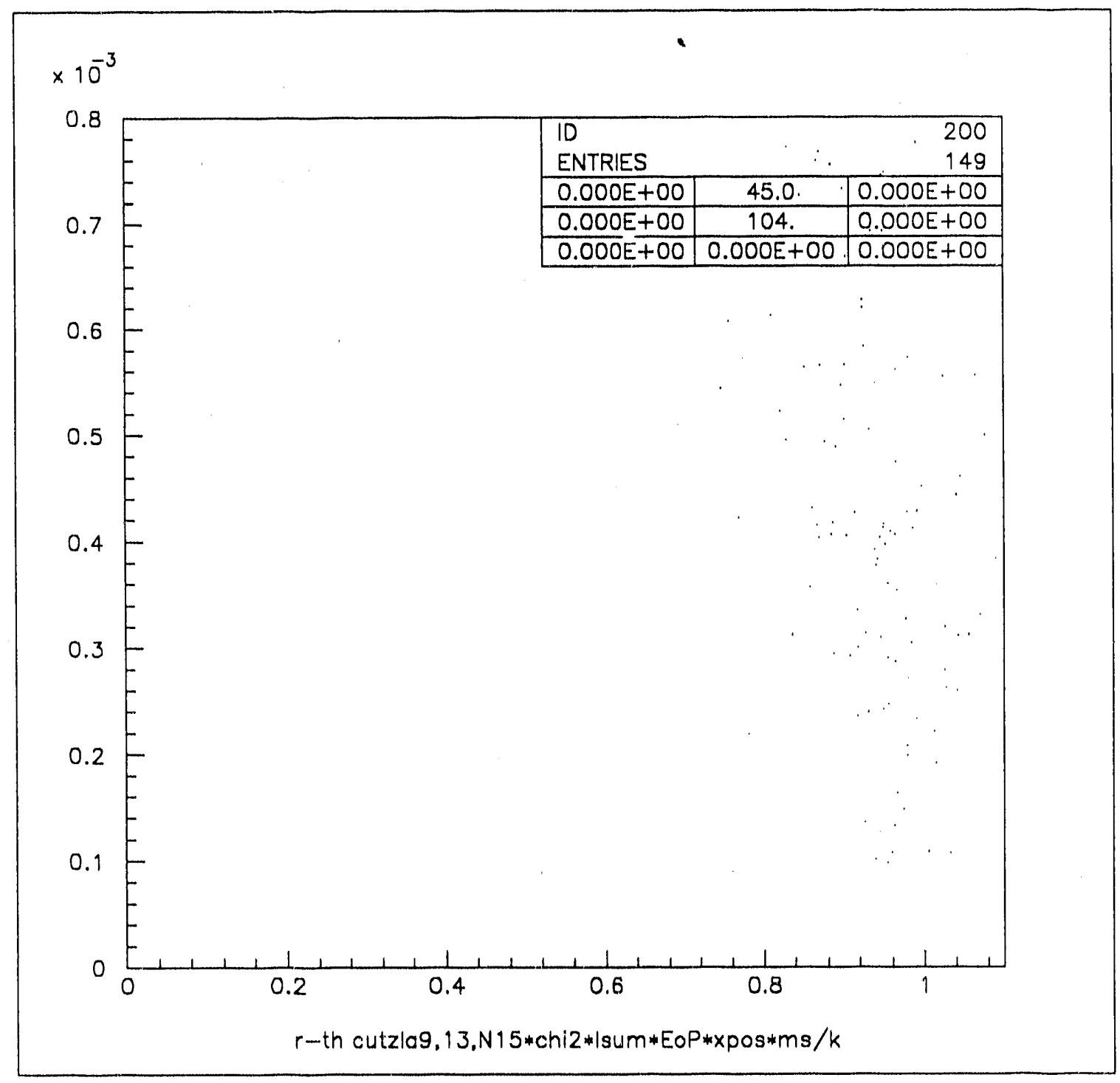

Figure 7. R-theta plot of events in Figure 6c. 


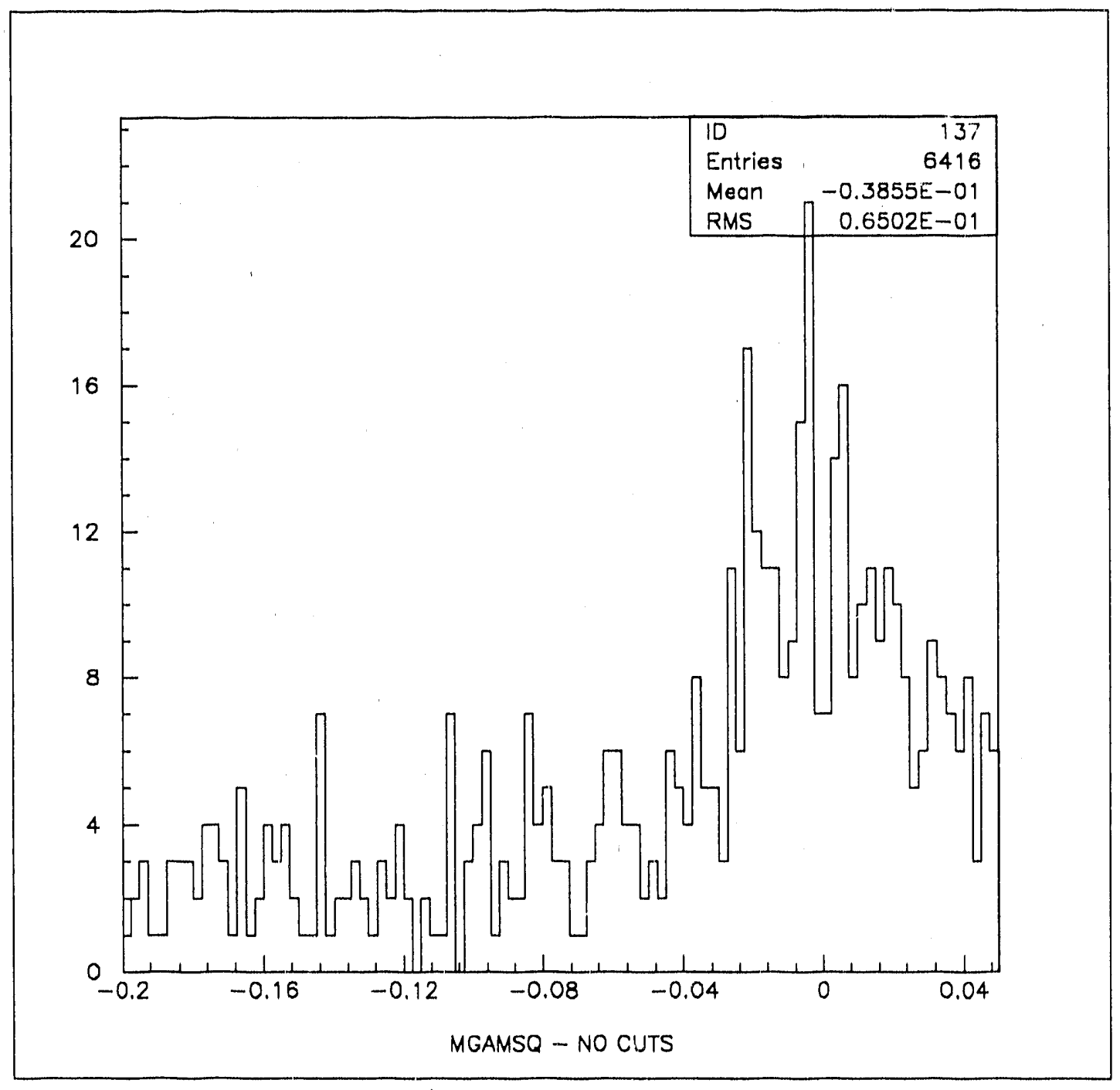

Figure 8 a Histograms of simulated fake cascade radiative decays produced by background $\pi^{0}$ production events. a. Mass-squared of the gamma with no cuts. b. Events selected to be similar to cascade radiative decay. 


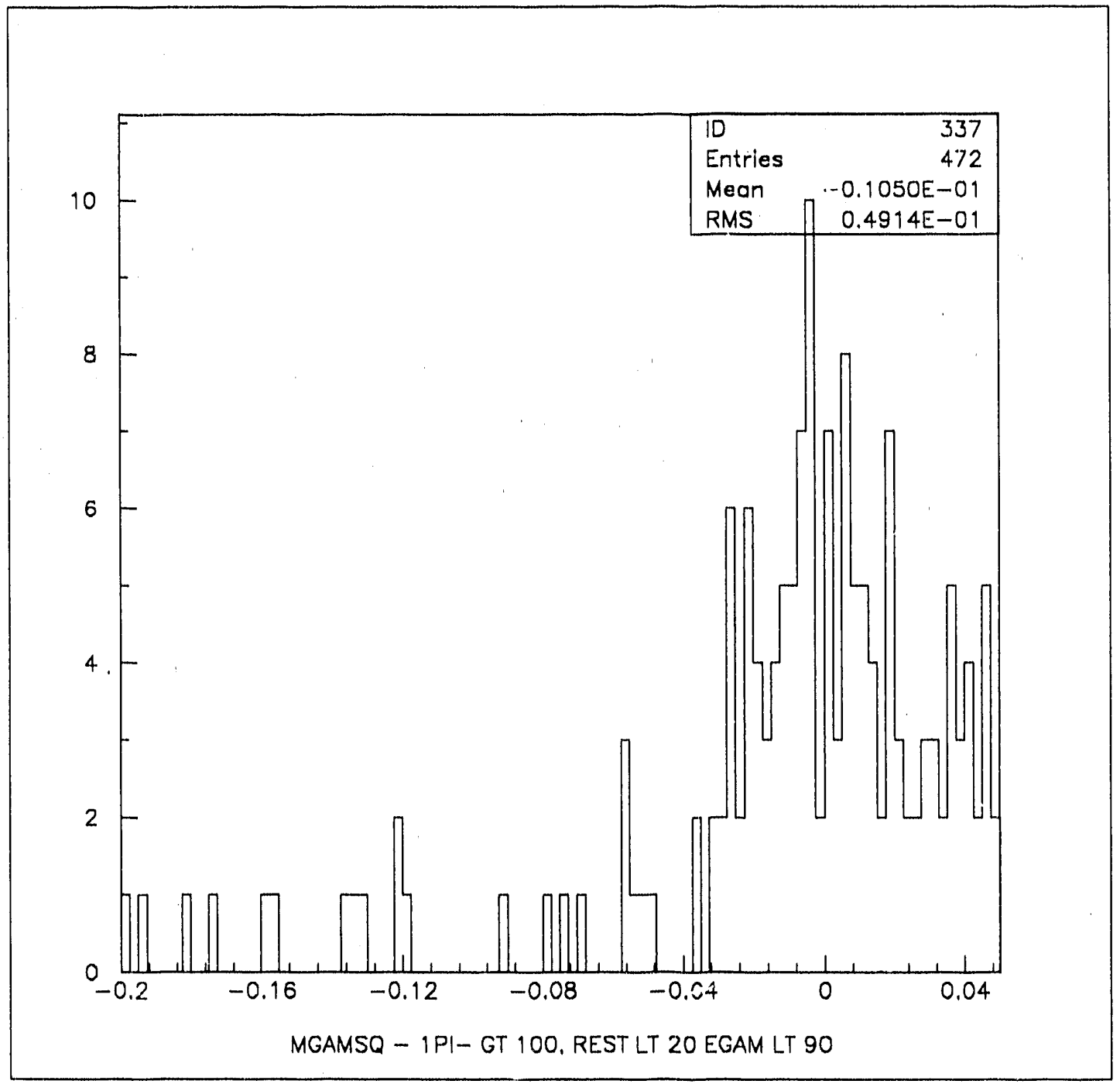

Figure $8 \mathrm{~b}$ Histograms of simulated fake cascade radiative decays produced ty background $\pi^{0}$ production events. a. Mass-squared of the gamma with no cuts. b. Events selected to be similar to cascade radiative decay. 


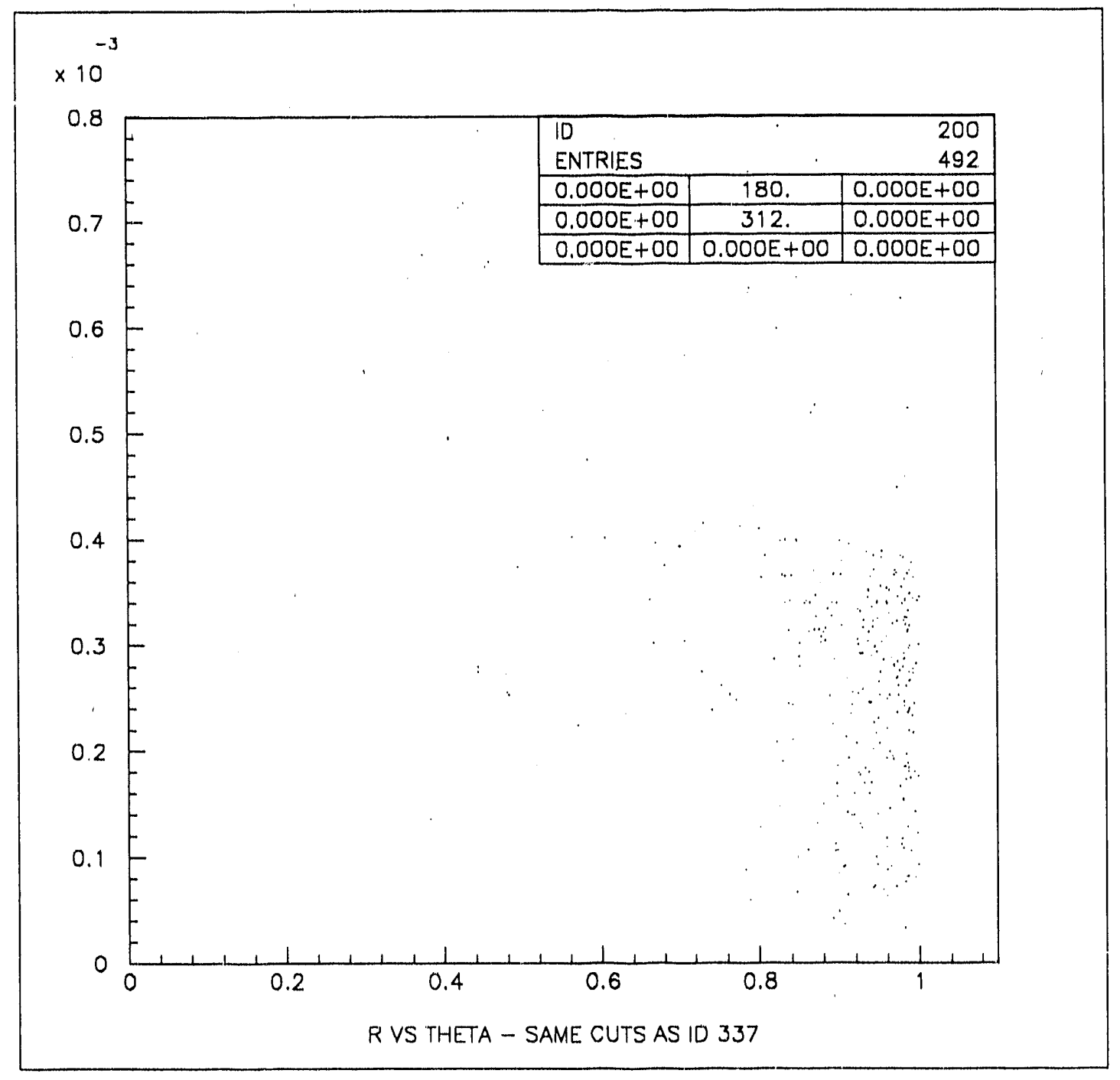

Figure 9. R-theta plot of events in Figure $8 \mathrm{~b}$. 


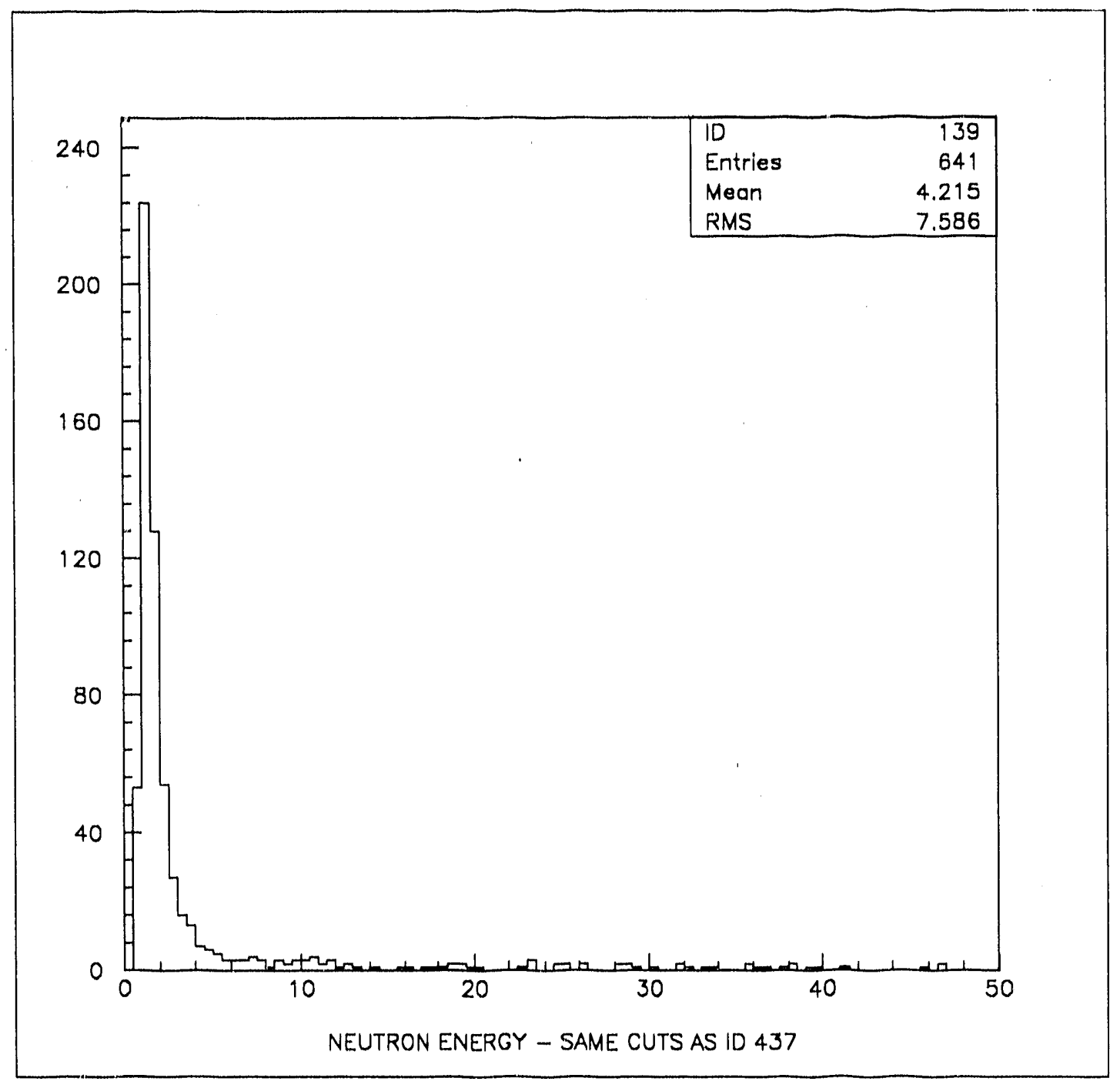

Figure 10. Neutron energy spectrum. Same events as Figure $8 \mathrm{~b}$. 


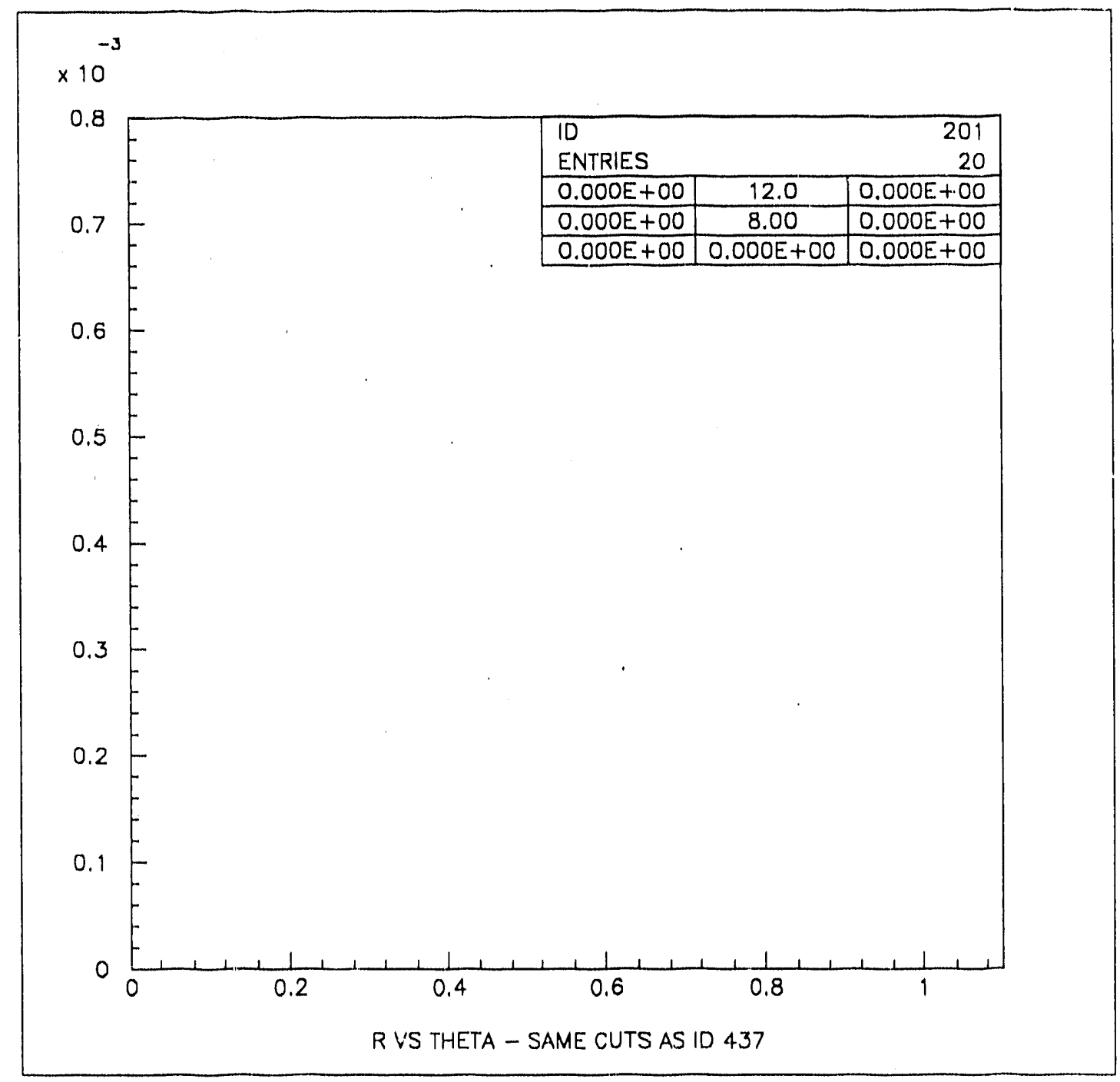

Figure 11. R-theta plot after neutron energy cut. 


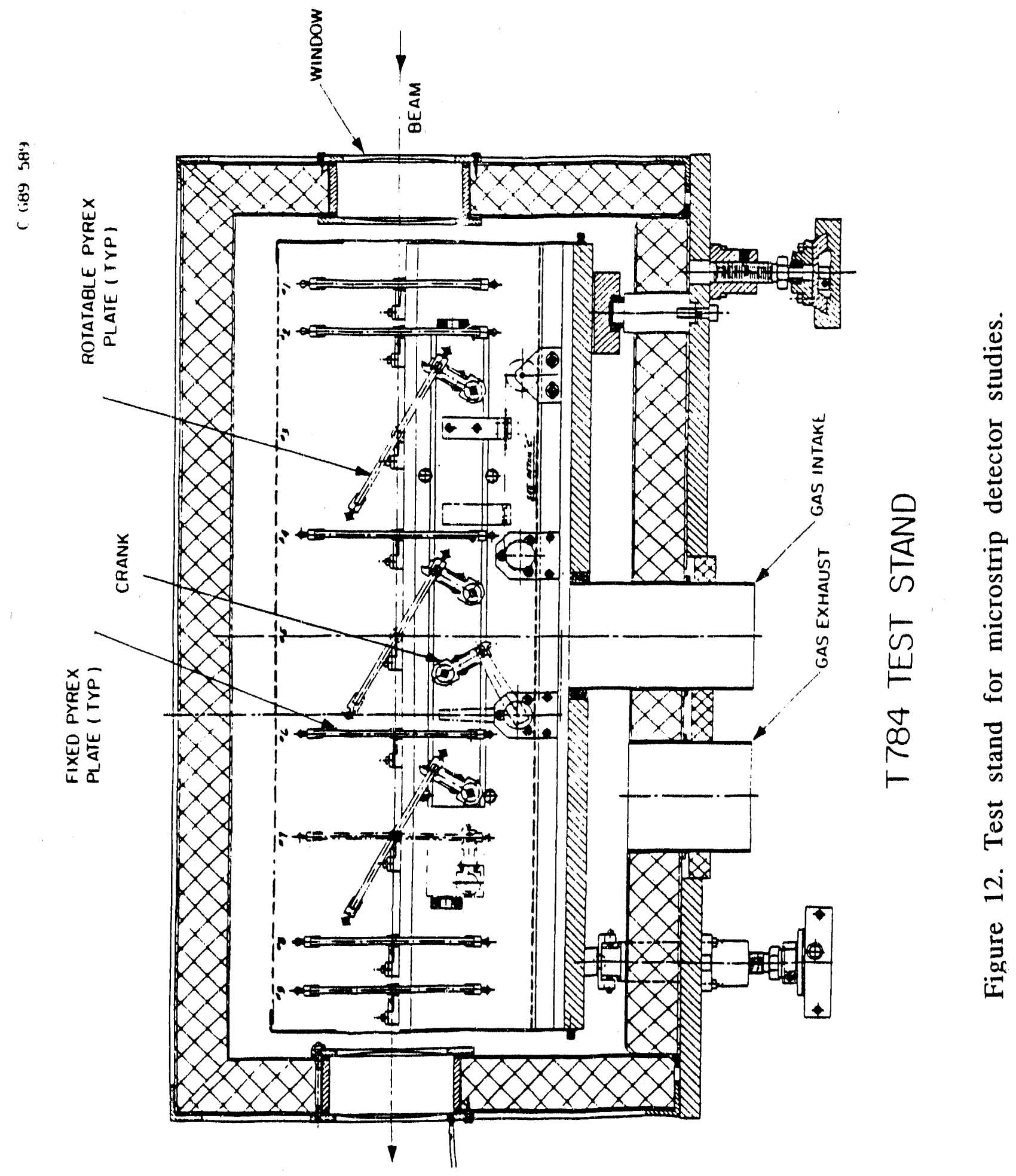




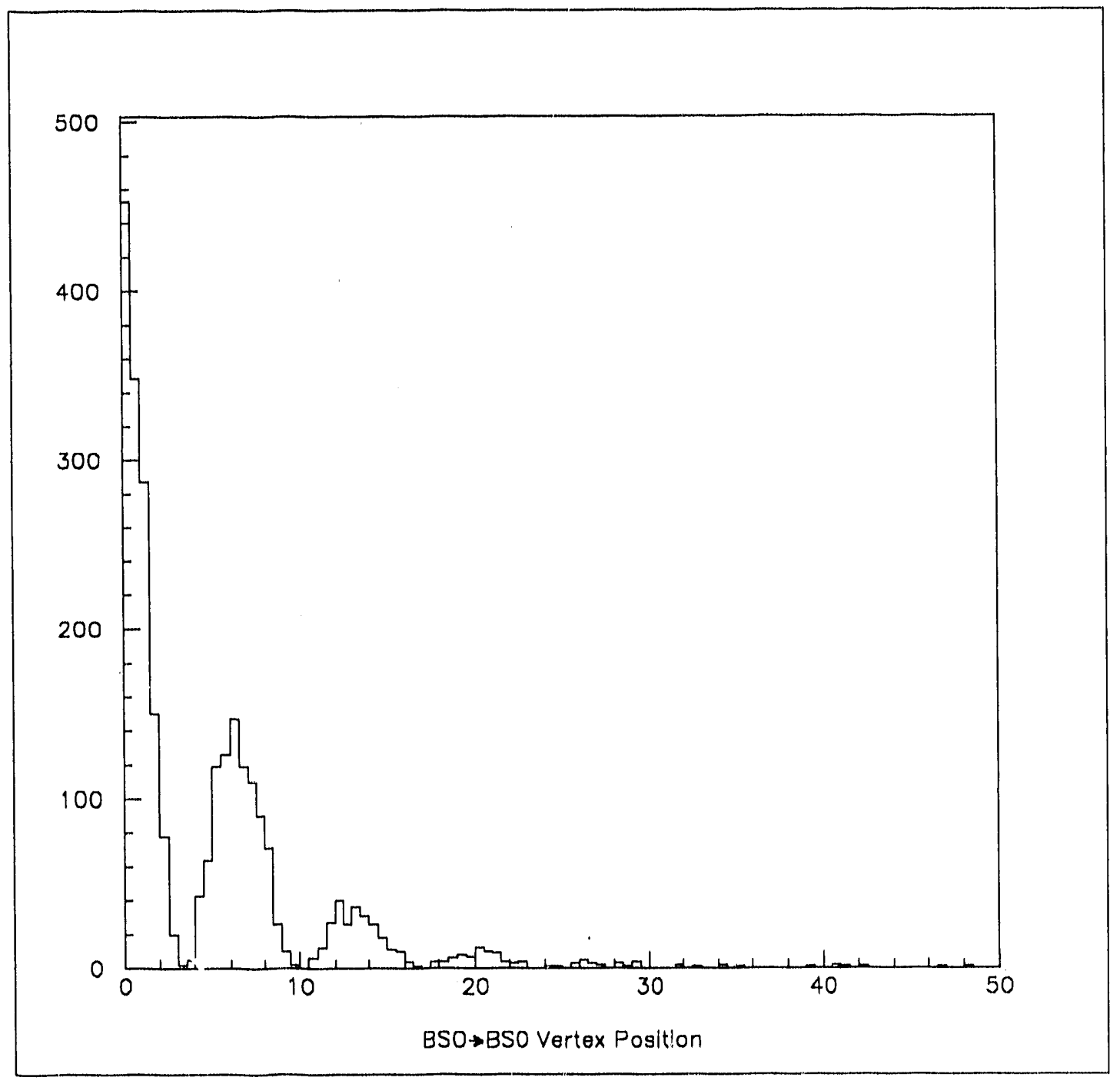

Figure 13 a Generated $B_{S} \rightarrow B_{S} B$ ar mixing as a function of $c \tau$ (arbitrary units), a. $B_{S} \rightarrow B_{S}$. b. $B_{S} \rightarrow B_{S} B$ ar. 


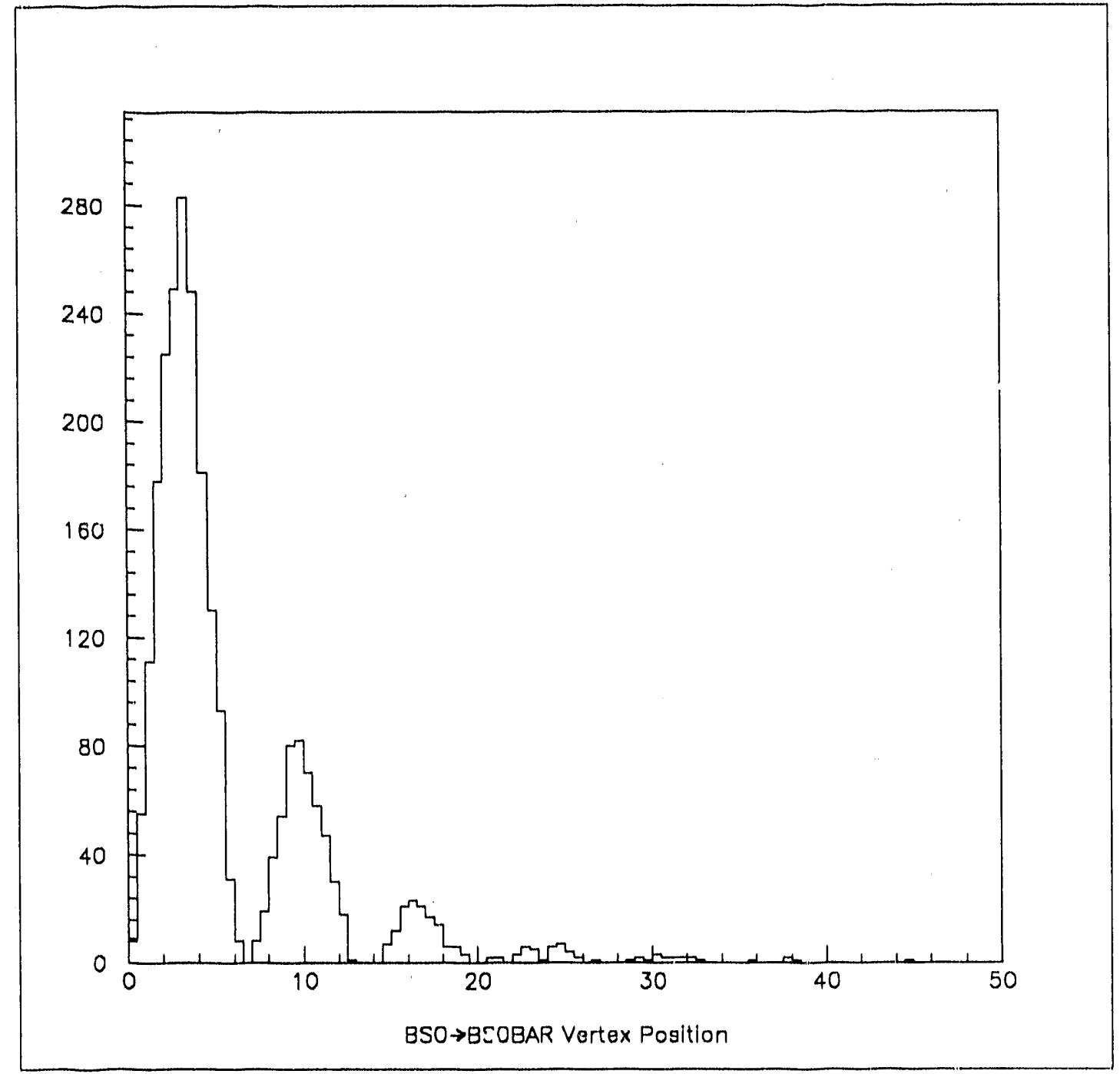

Figure $13 \mathrm{~b}$ Generated $\mathrm{B}_{S}>\mathrm{B}_{\mathrm{S}} \mathrm{Bar}$ mixing as a function of $\mathrm{c} \tau$ (arbitrary units). a. $B_{S} \rightarrow B_{S}$. b. $B_{S} \rightarrow B_{S} B$ ar. 


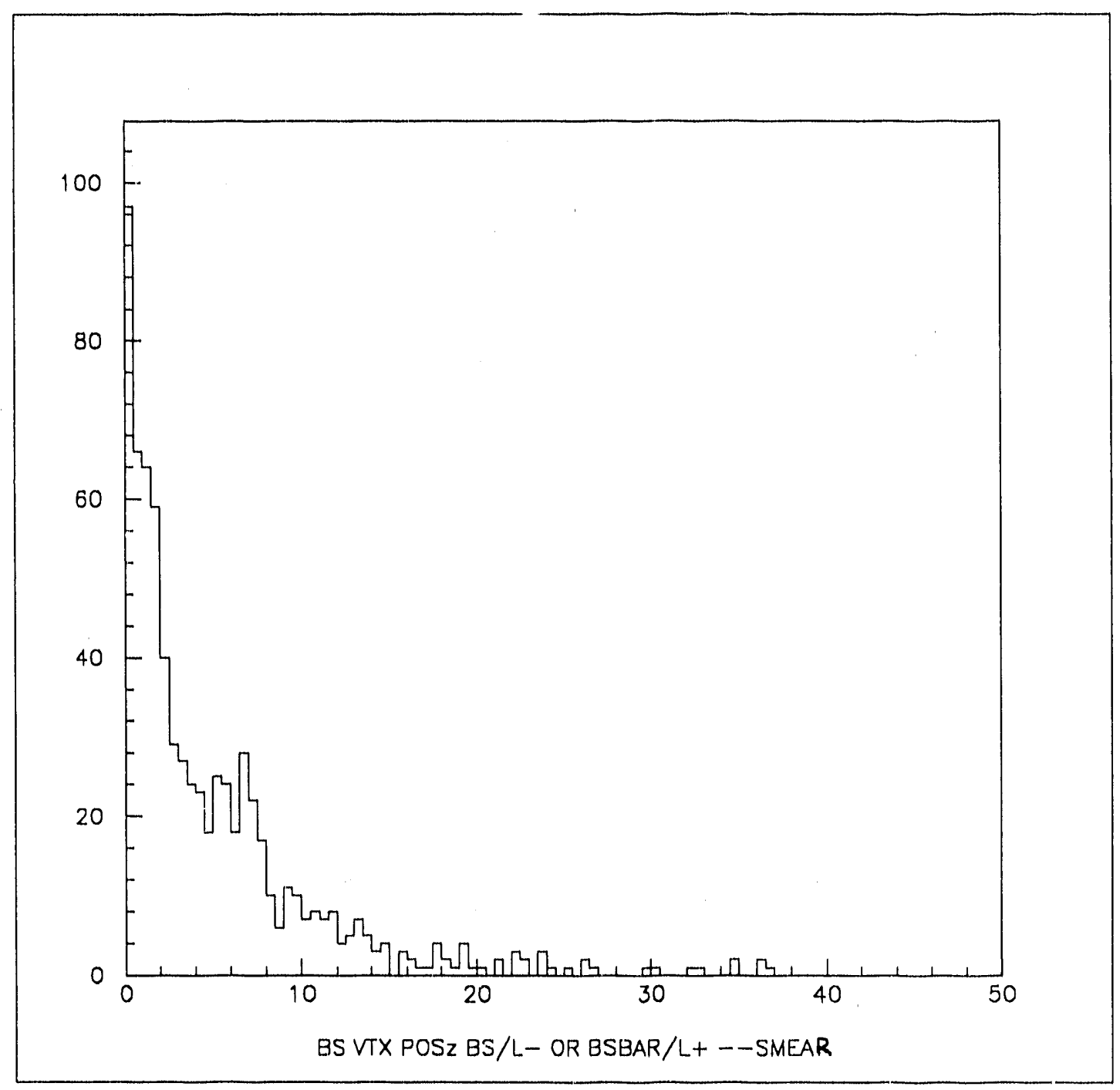

Figure 14 a Histograms of simulated vertex positions of "detected" leptons from $a$. unmixed $B_{S}$ 's or $B_{S} B$ ars and $b$. mixed $B_{S}$ 's or BsBars. 


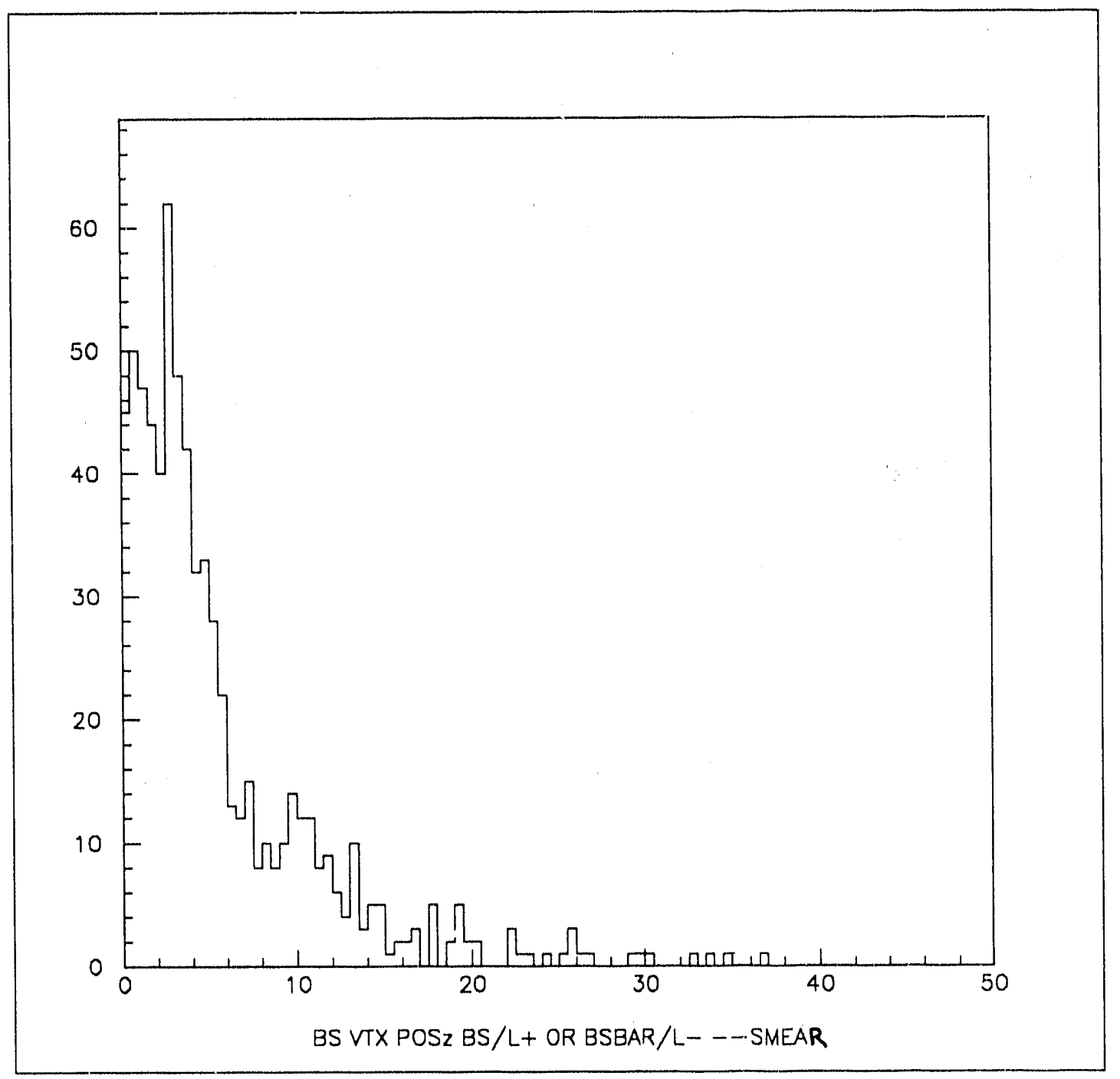

Figure $14 \mathrm{~b}$ Histograms of simulated verrex positions of "detected" leptons from a. unmixed $B_{s}$ 's or $B_{S} B$ ars, and $b$. mixed $B_{S}$ 's or Bs Bars. 


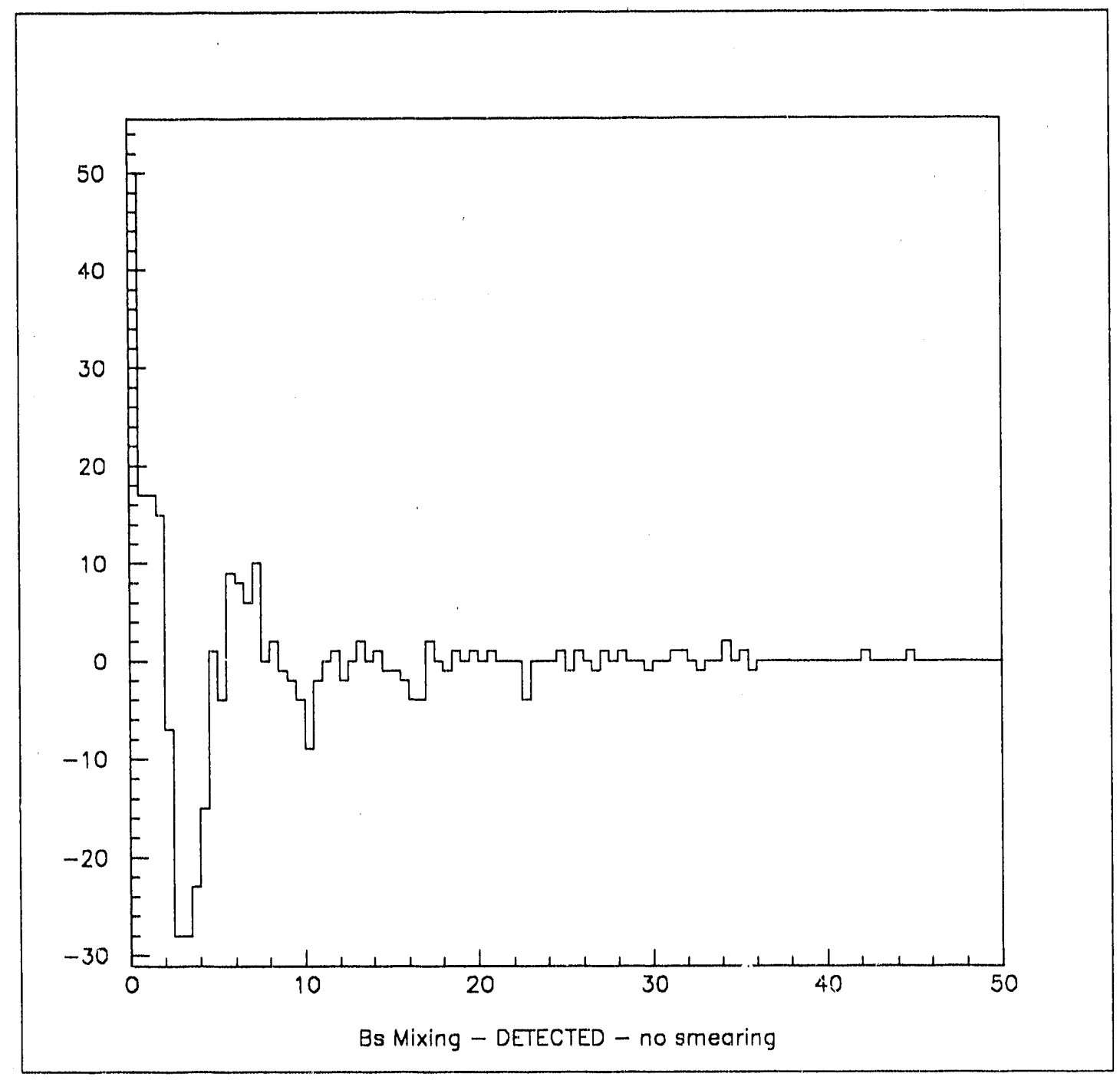

Figure 15. Subtracted histogram, showing mixing. 


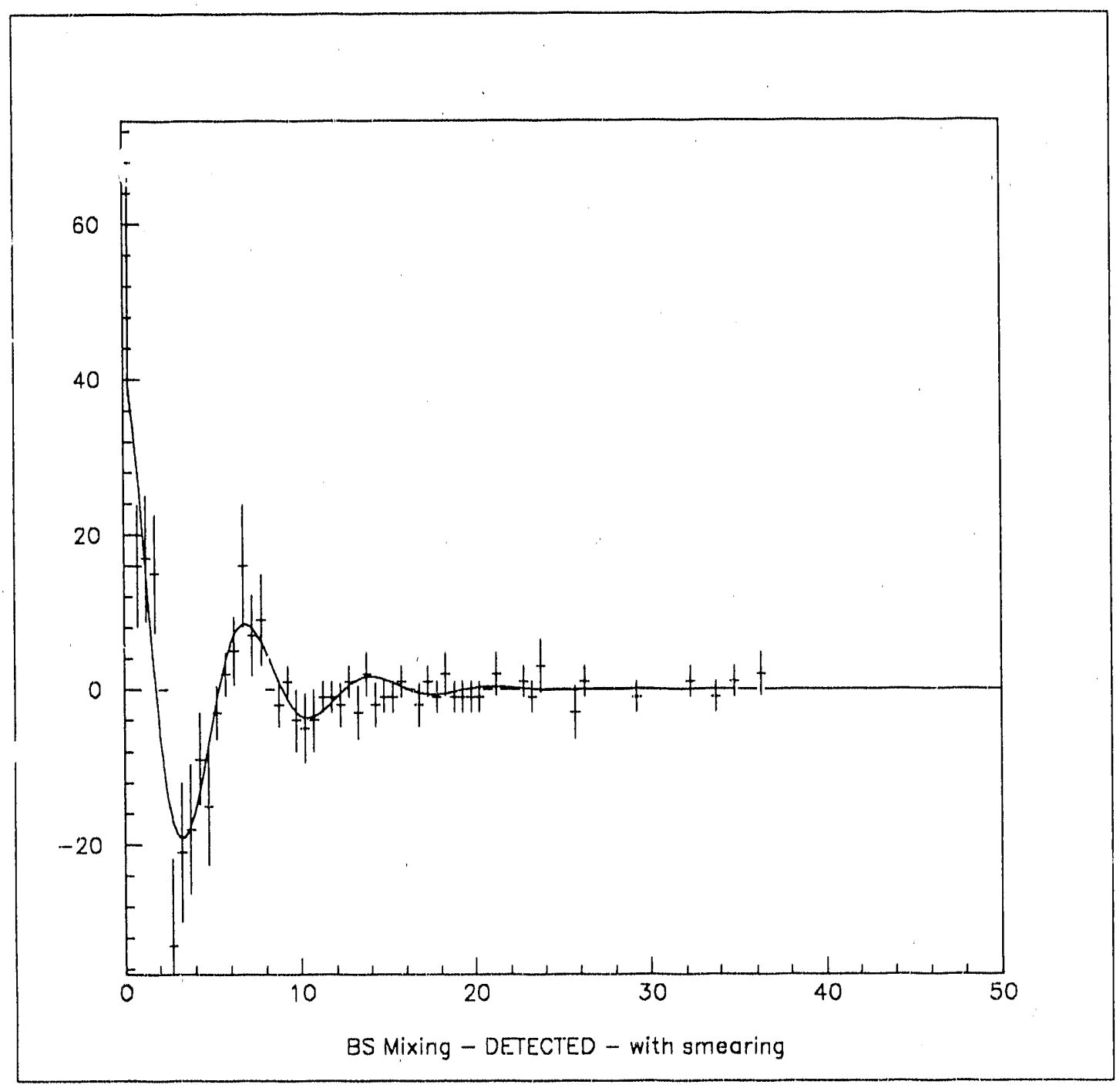

Fi.gure $16 \mathrm{a}$ Fits to mixing histograms. a. Mixing parameter $\mathrm{x}_{\mathbf{S}}=5.0$.

b. Mixing parameter $x_{S}=10.0$. c. Mixing parameter $x_{S}=20.0$. 


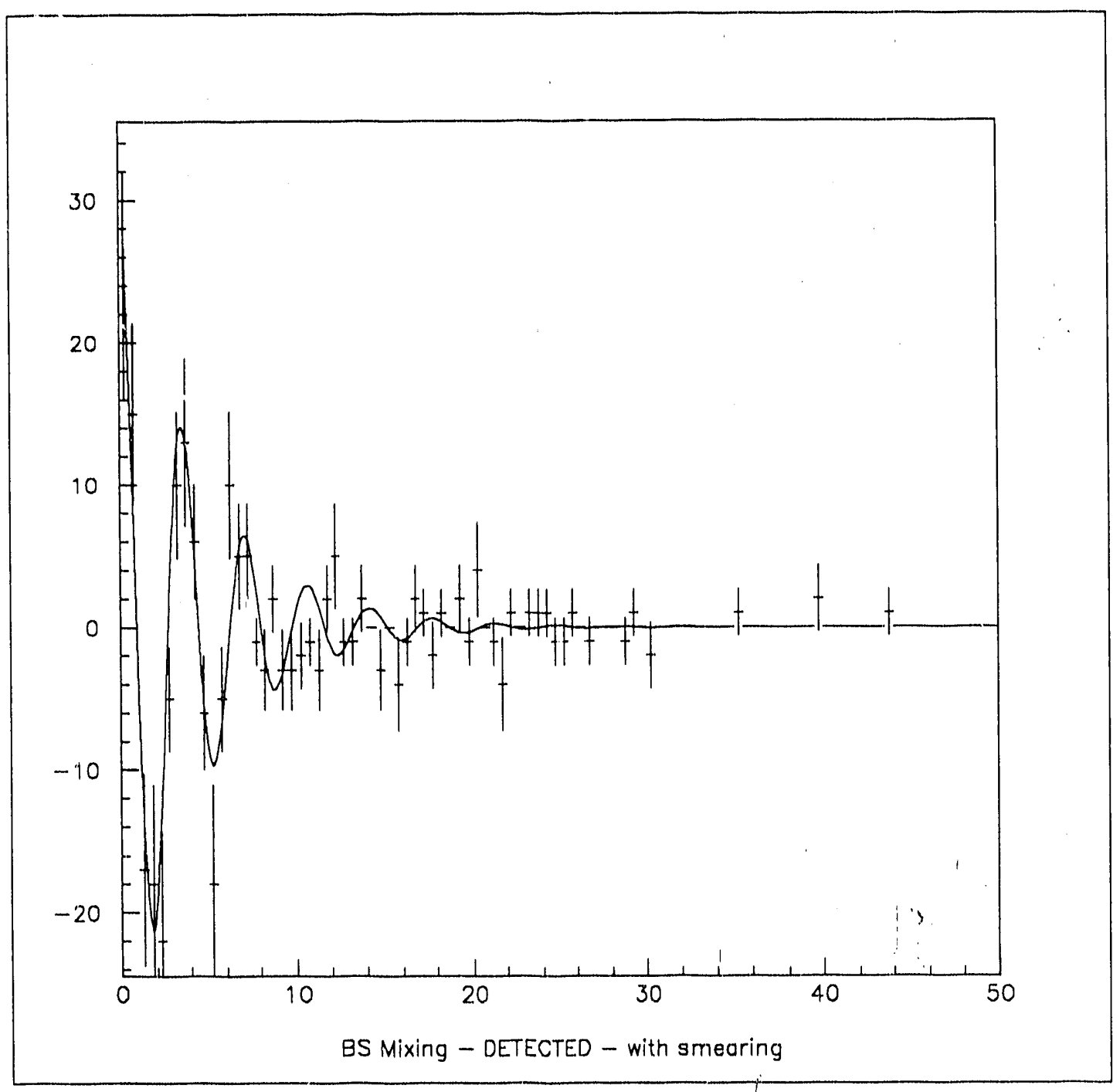

Figure $16 \mathrm{~b}$ Fits to mixing histograms. a. Mixing parameter $\mathrm{x}_{\mathrm{S}}=5.0$. b. Mixing parameter $x_{S}=10.0$. c. Mixing parameter $x_{S}=20.0$. 


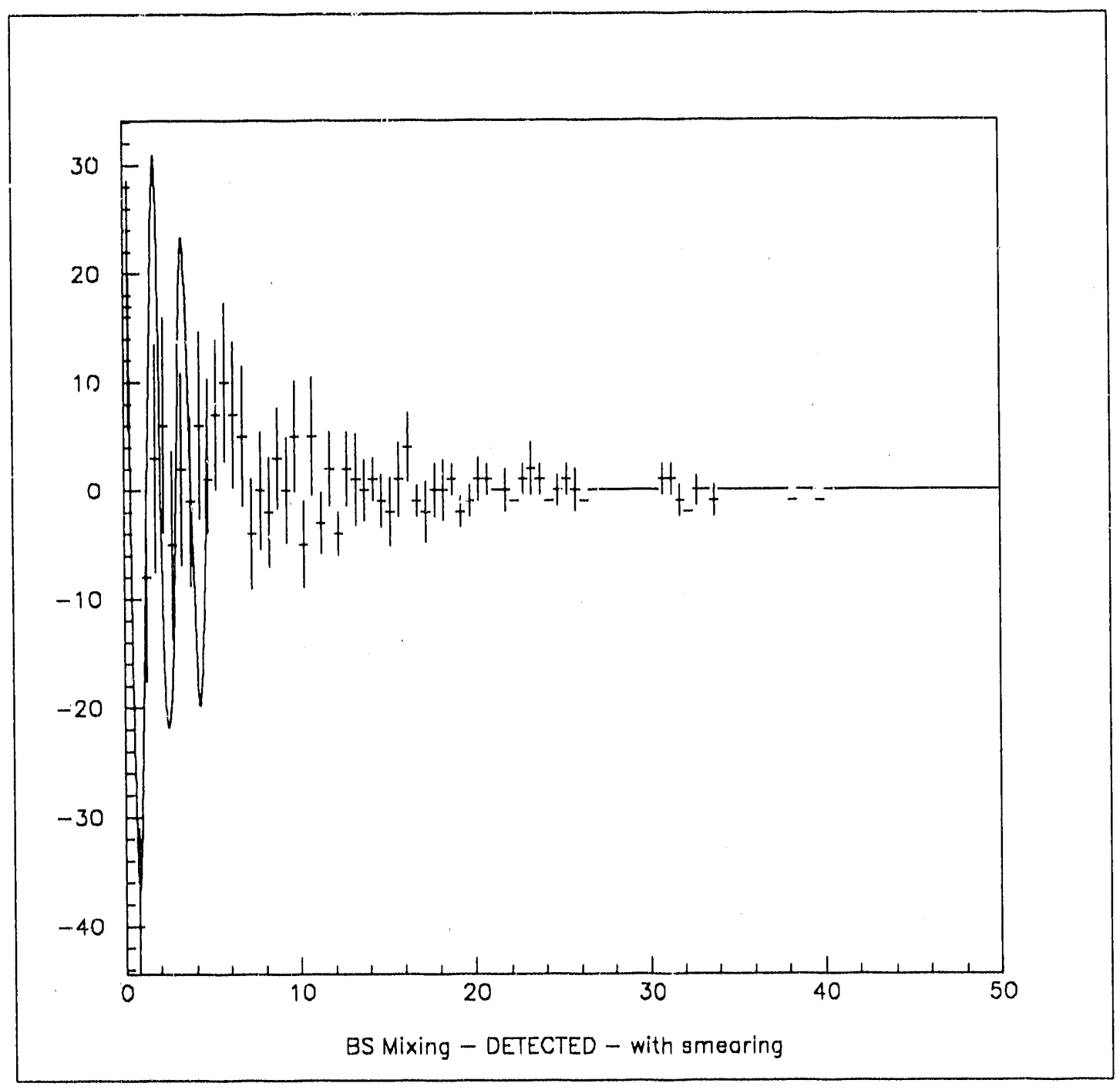

Eigure $16 \mathrm{C}$ Fits to mixing histograms. a. Mixing parameter $\mathrm{x}_{\mathrm{S}}=5.0$. b. Mixing parameter $x_{s}=10.0$. c. Mixing parameter $x_{s}=20.0$. 


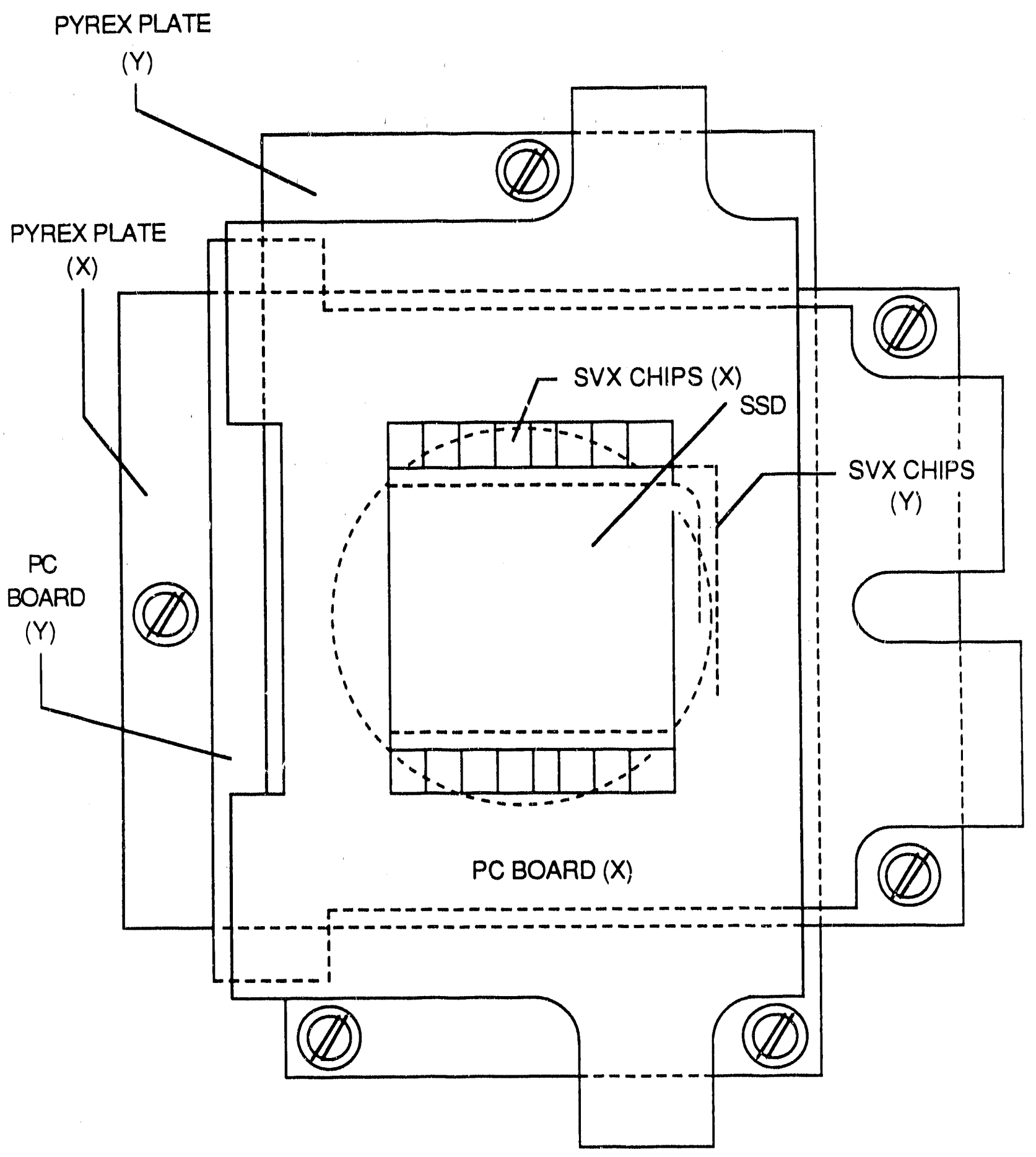

E781 SILICON STRIP DETECTOR ASSEMBLY

Figure 17. A ship carrier assembly for experiment E781. 


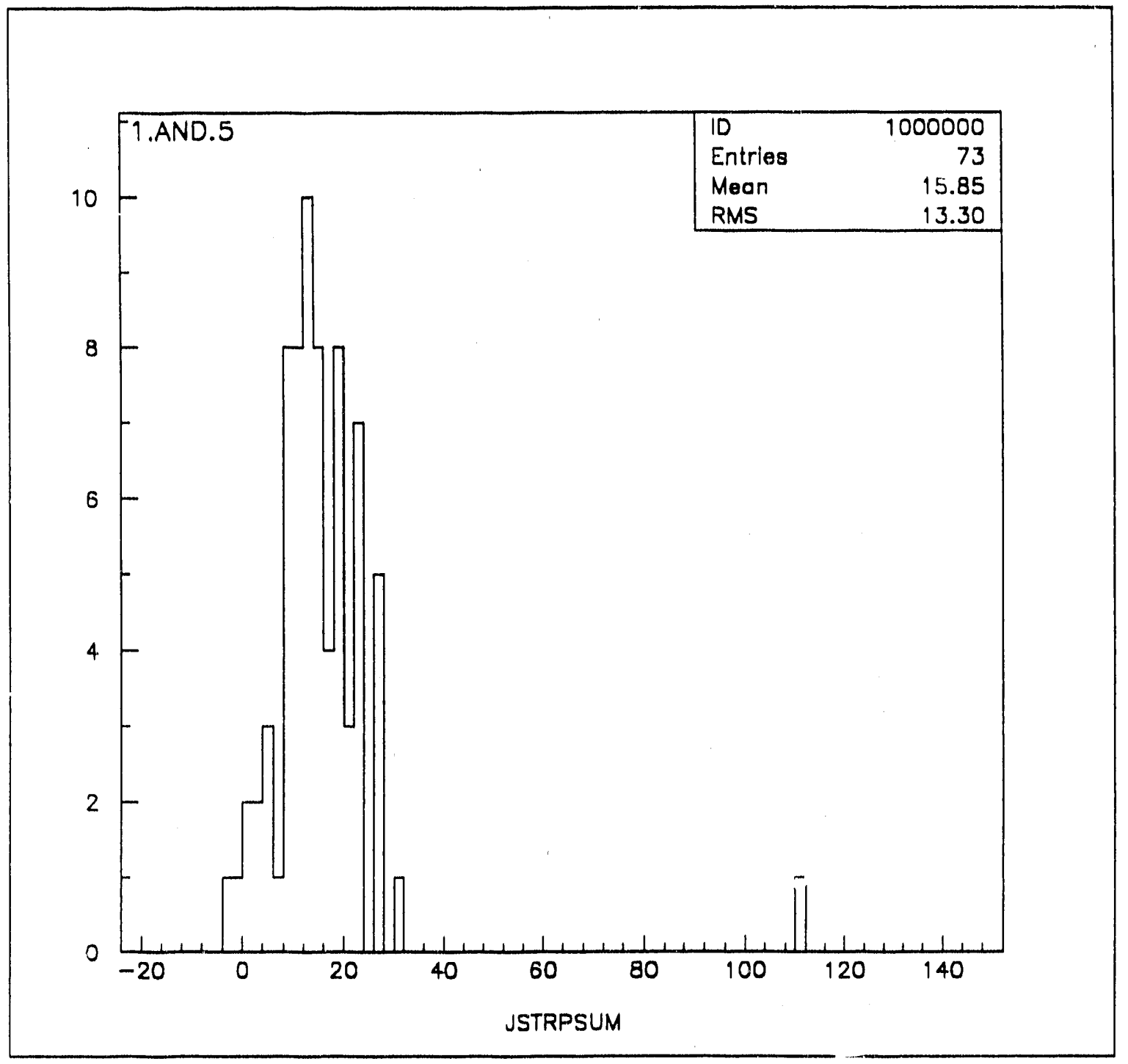

Figure 18. Distribution of charge detected with a working strip detector using SVXD microchip readout. Each channel corresponds to $0.25 \mathrm{fC}$. 

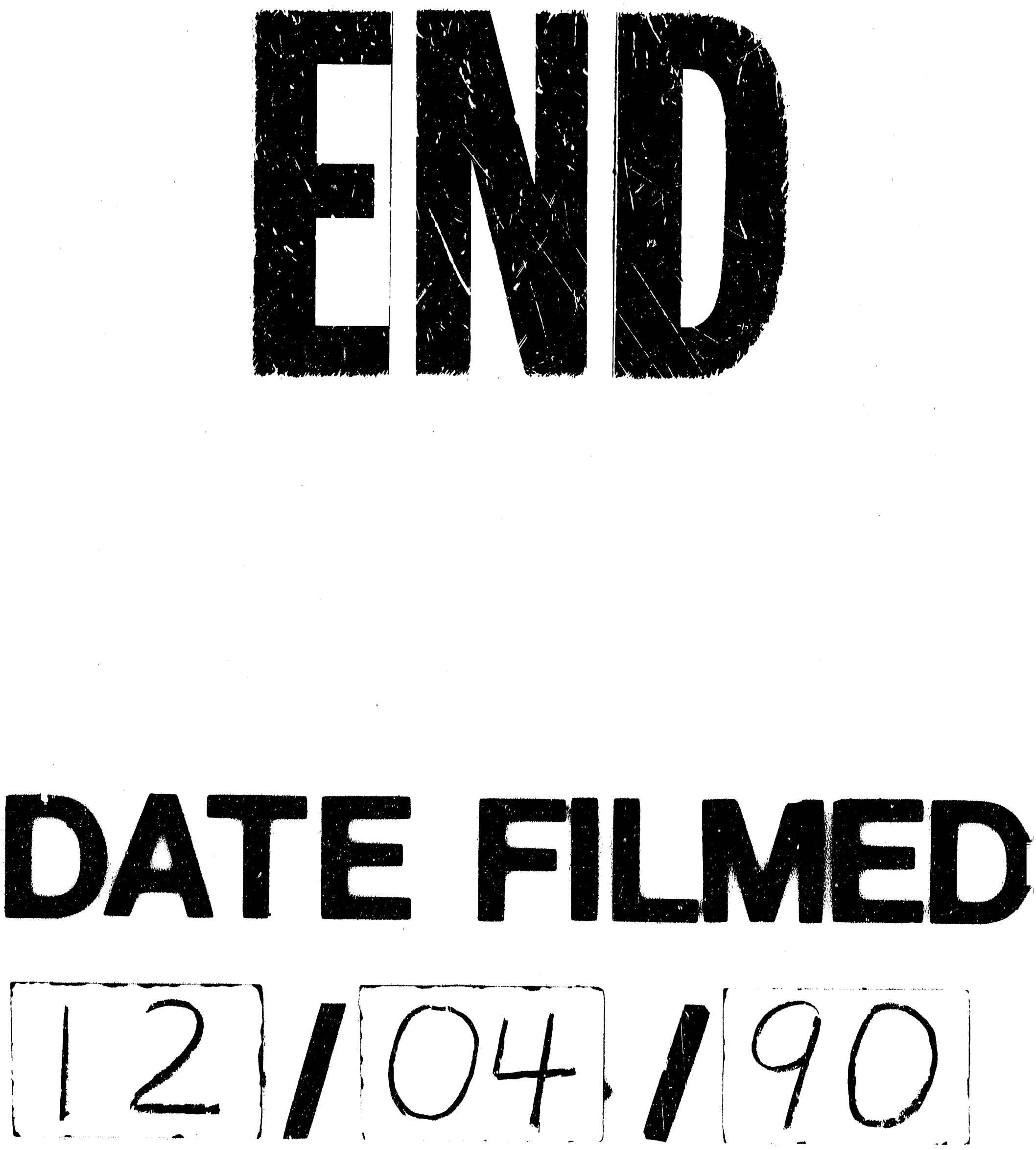
